\title{
Topological and analytical properties of Sobolev bundles. II. Higher dimensional cases
}

Takeshi Isobe

\begin{abstract}
We define various classes of Sobolev bundles and connections and study their topological and analytical properties. We show that certain kinds of topologies (which depend on the classes) are well-defined for such bundles and they are stable with respect to the natural Sobolev topologies. We also extend the classical Chern-Weil theory for such classes of bundles and connections. Applications related to variational problems for the Yang-Mills functional are also given.
\end{abstract}

\section{Introduction}

This paper is a sequel to [13] where we have studied an interrelation between the topological and the analytical properties of Sobolev bundles and Sobolev connections for the critical case.

Let us first recall the definition of Sobolev bundles as introduced in [13]. Throughout this paper, we assume that $M$ is a compact Riemannian manifold of dimension $m$ and $G$ a compact Lie group with bi-invariant metric. There is a faithful unitary representation of $G$ on $\mathbb{R}^{l}$, i.e., an injection $G \rightarrow O\left(\mathbb{R}^{l}\right)=O(l)$ for some $l \in \mathbb{Z}$. Thus we may assume without loss of generality that $G$ is a subgroup of $O(l)$. For any open subset $U \subset M, k \in \mathbb{Z}$ with $k \geq 1$ and $1 \leq p \leq \infty$, the Sobolev space of functions in $U$ of class $W^{k, p}$, denoted $W^{k, p}(U)$, is defined as the set of all $L^{p}$-integrable functions in $U$ whose partial derivatives (in the sense of distributions) of order up to $k$ are also $L^{p}$-integrable in $U$. $W^{k, p}(U, G)$ is then defined as

$$
W^{k, p}(U, G)=\left\{g \in W^{k, p}\left(U, \mathbb{R}^{l^{2}}\right): g(x) \in G \text { for a.e. } x \in U\right\} .
$$

2000 Mathematics Subject Classification: 46E35, 57R22, 58E15.

Keywords: Sobolev bundle, topology of bundle, Yang-Mills functional, variational problem. 
By the Sobolev embedding theorem, if $k$ and $p$ satisfy $k p>m$, then $W^{k, p}(U, G) \subset C^{0}(\bar{U}, G)$ and the pointwise multiplication and the inversion define a continuous multiplication $W^{k, p}(U, G) \times W^{k, p}(U, G) \ni(f, g) \mapsto f \cdot g \in$ $W^{k, p}(U, G)$ and an inversion $W^{k, p}(U, G) \ni f \mapsto f^{-1} \in W^{k, p}(U, G)$. With these, $W^{k, p}(U, G)$ becomes a Banach Lie group. This is not the case for $k p \leq m$, but by the Gagliardo-Nirenberg inequality, in [13] we have shown that it becomes a topological group under the pointwise multiplication and the inversion even for the case $k p \leq m$.

Classical (topological or smooth) principal $G$-bundles are described by an open covering $\left\{U_{\alpha}\right\}$ of $M$ and a gluing date $\left\{g_{\alpha \beta}\right\}, g_{\alpha \beta} \in C^{0}\left(U_{\alpha \beta}, G\right)$ (or $C^{\infty}\left(U_{\alpha \beta}, G\right)$ when considering smooth bundles) which define an element in $\check{H}^{1}\left(M, \mathfrak{C}_{G}^{0}\right)$ (respectively, $\check{H}^{1}\left(M, \mathcal{C}_{G}^{\infty}\right)$ ), where $U_{\alpha \beta}=U_{\alpha} \cap U_{\beta}$ and $\mathfrak{C}_{G}^{0}$ (respectively, $\mathcal{C}_{G}^{\infty}$ ) denotes a presheaf defined by $\mathcal{C}_{G}^{0}(U)=C^{0}(U, G)$ (respectively, $\left.\mathrm{C}_{G}^{\infty}(U)=C^{\infty}(U, G)\right)$ for any open set $U \subset M$. Conversely, any element in $\check{H}^{1}\left(M, \mathrm{C}_{G}^{0}\right)$ (or $\check{H}^{1}\left(M, \mathrm{C}_{G}^{\infty}\right)$ ) defines a unique isomorphism class of principal $G$-bundles of class $C^{0}$ (respectively, $C^{\infty}$ ). The Sobolev bundles of class $W^{k, p}$ are defined similarly. Namely, it is defined as an element of $\check{H}^{1}\left(M, \mathcal{W}_{G}^{k, p}\right)$, where the presheaf $\mathcal{W}_{G}^{k, p}$ is defined by $\mathcal{W}_{G}^{k, p}(U)=W^{k, p}(U, G)$ for any open subset $U \subset M$. In other words, it is described as a pair $P=\left\langle\left\{U_{\alpha}\right\}_{\alpha \in I},\left\{g_{\alpha \beta}\right\}_{\alpha \beta \in I}\right\rangle$, where $\left\{U_{\alpha}\right\}_{\alpha \in I}$ is an open covering of $M$ and $g_{\alpha \beta} \in W^{k, p}\left(U_{\alpha \beta}, G\right)(\alpha, \beta \in I)$ satisfy $g_{\alpha \gamma}(x)=g_{\alpha \beta} \cdot g_{\beta \gamma}(x)$ for a.e. $x \in$ $U_{\alpha \beta \gamma}:=U_{\alpha} \cap U_{\beta} \cap U_{\gamma}$. For more details, see [13]. Notice that, by the Sobolev embedding theorem, Sobolev bundles of class $W^{k, p}$ are in fact bundles of class $C^{0}$ when $k p>m$. Thus for our study the interesting cases are $k p \leq m$ and we only treat these cases throughout this paper.

Sobolev bundles naturally arise as limits of smooth bundles. For example, if we want to compactify the moduli space of (smooth) Yang-Mills connections under the natural topology, one needs to add weak limiting bundles and connections on them. These weak limiting objects are, in general, singular and belong to certain classes of Sobolev bundles and connections. There are also other places where such bundles and connections arise: For example, if we want to apply techniques of the calculus of variations for the Yang-Mills and other related functionals, one needs to work on a suitable Sobolev completion of smooth bundles and connections in order to get suitable compactness properties. For more details and other examples, see [27], [38], [39], [40], [36] [24] and [13].

In our previous paper [13], we studied an interplay between the topological and the analytical properties of Sobolev bundles and connections in the critical case $k p=m$. One of our results proved there is the equivalence of the category of principal $G$-bundles of class $W^{k, p}$ and the category of principal $G$-bundles of class $C^{0}$, i.e., the isomorphism $H^{1}\left(M, \mathcal{W}_{G}^{k, p}\right) \cong H^{1}\left(M, \mathrm{C}_{G}^{0}\right)$. This answers the question raised by Uhlenbeck in her paper [40]. 
In this paper, we continue our study and concentrate on our attention to the higher dimensional cases $k p<m$. Contrary to the critical and the lower dimensional cases $k p \geq m$, it is possible that one can define various classes of Sobolev bundles and connections with the same indexes $k, p$. This is due to the fact that there are various (inequivalent) classes of Sobolev spaces with values into $G$ when $k p<m$. So we first define these classes in $\S 2$. In the higher dimensional case, one can not expect the equivalence of the category of Sobolev $G$-bundles to the category of $G$-bundles of class $C^{0}$. However, we shall show that certain kinds of topological invariants are well-defined for such bundles. These are defined in $§ 3$. Its properties, in particular, its stability with respect to the appropriate Sobolev topologies are also studied in $\S 3$.

On Sobolev bundles, there are also natural classes of Sobolev connections. These are defined in $\S 2$. For these connections, in $\S 4$, we extend the classical Chern-Weil theory and study its compatibility with the topologies introduced in $\S 3$. In $\S 5$, we introduce singular Sobolev bundles which also naturally arise as weak limits of smooth bundles. The results in $\S 3$ and $\S 4$ are extended for such classes of bundles. As an application of the results proved in $\S 3$ and $\S 5$, we consider $G$-bundles with Neather Yang-Mills connections (see $\S 5$ for the definition) which have uniformly bounded Yang-Mills energies. We shall show that such bundles converge weakly to a certain class of (singular) Sobolev bundles. We characterize these limits in terms of the invariants defined in $\S 3$ and $\S 5$.

In $\S 6$, we study variational problems for the Yang-Mills functional. In that section, we characterize the value of the infimum of the Yang-Mills functional in terms of the invariants introduced in $\S 3$.

Our results are considered as Yang-Mills counterparts of the results obtained by White [41], [42] (see also [10]) for mapping problems in Sobolev spaces. And we use some of the ideas of [41], [42] and [10] in our proof of the results.

\section{Various classes of Sobolev bundles and Sobolev con- nections}

\subsection{Classes of Sobolev bundles}

Let $k \in \mathbb{N}$ and $p \geq 1$ be such that $k p<m$. As mentioned in the introduction, there are various classes of Sobolev spaces defined on an open subset $U \subset M$ with values in $G$. One natural definition is given by (1.1). However, other choices are also possible. These are obtained as completions of $C^{\infty}(U, G) \cap W^{k, p}(U, G)$ with respect to natural Sobolev topologies. 
One is obtained as the completion of $C^{\infty}(U, G) \cap W^{k, p}(U, G)$ (or equivalently $\left.C^{0}(U, G) \cap W^{k, p}(U, G)\right)$ with respect to the strong Sobolev topology (i.e., the topology defined by the Sobolev norm). We denote this as $W_{\text {strong }}^{k, p}(U, G)$ :

$W_{\text {strong }}^{k, p}(U, G)=$ the strong closure of $C^{\infty}(U, G) \cap W^{k, p}(U, G)$ in $W^{k, p}(U, G)$.

Another natural space is the sequential weak closure of $C^{\infty}(U, G) \cap W^{k, p}(U, G)$ (or equivalently $C^{0}(U, G) \cap W^{k, p}(U, G)$ ) in $W^{k, p}(U, G)$ which we denote by $W_{\text {weak }}^{k, p}(U, G)$ :

$$
\begin{aligned}
& W_{\text {weak }}^{k, p}(U, G)= \\
& =\text { the sequential weak closure of } C^{\infty}(U, G) \cap W^{k, p}(U, G) \text { in } W^{k, p}(U, G) .
\end{aligned}
$$

For any open set $U \subset M, W^{k, p}(U, G), W_{\text {strong }}^{k, p}(U, G)$ and $W_{\text {weak }}^{k, p}(U, G)$ are very different when $k p<m$. (They define the same set when $k p \geq m$ due to the approximation theorem of Schoen-Uhlenbeck [26]). In fact, when $\pi_{[k p]}(G) \neq 0$ we always have

$$
W_{\text {strong }}^{k, p}(U, G) \varsubsetneqq W^{k, p}(U, G)
$$

in view of the result of Bethuel [1] and Bethuel-Zheng [2] and we also have

$$
W_{\text {strong }}^{k, p}(U, G) \varsubsetneqq W_{\text {weak }}^{k, p}(U, G)
$$

in general (see [1], [10]). According to the different choices of Sobolev spaces, we obtain different kinds of (the isomorphism classes of) Sobolev bundles. They are defined as

$$
\check{H}^{1}\left(M, \mathcal{W}_{G}^{k, p}\right), \quad \check{H}^{1}\left(M, \mathcal{W}_{\text {strong, },}^{k, p}\right) \quad \text { and } \quad \check{H}^{1}\left(M, \mathcal{W}_{\text {weak }, G}^{k, p}\right)
$$

respectively, where $\mathcal{W}_{\text {strong,G }}^{k, p}$ and $\mathcal{W}_{\text {weak,G }}^{k, p}$ are presheaves defined by

$$
\mathcal{W}_{\text {strong,G }}^{k, p}(U)=W_{\text {strong }}^{k, p}(U, G) \quad \text { and } \quad \mathcal{W}_{\text {weak }, G}^{k, p}(U)=W_{\text {weak }}^{k, p}(U, G)
$$

respectively, for any open set $U \subset M$. To see that these definitions are meaningful, we need to show that $W^{k, p}(U, G), W_{\text {strong }}^{k, p}(U, G)$ and $W_{\text {weak }}^{k, p}(U, G)$ become groups with respect to the pointwise multiplication and the inversion. This is seen from the Gagliardo-Nirenberg inequality as in [13], see also below. Though the above classes of Sobolev bundles are natural, there are also other natural choices: Since in applications we frequently encounter classes of Sobolev bundles as obtained by limits of smooth bundles with respect to suitable Sobolev topologies, we are in fact interested in some subsets of $\check{H}^{1}\left(M, \mathcal{W}_{\text {strong, },}^{k, p}\right)$ and $\check{H}^{1}\left(M, \mathcal{W}_{\text {weak }, G}^{k, p}\right)$ defined below: 
Definition 2.1 Let $k \in \mathbb{N}$ and $1 \leq p \leq \infty$.

(1) The class of Sobolev bundles $\mathcal{P}_{G}^{k, p}(M)$ is defined as the set of pairs $P=\left\langle\left\{U_{\alpha}\right\}_{\alpha \in I},\left\{g_{\alpha \beta}\right\}_{\alpha, \beta \in I}\right\rangle$, where $\left\{U_{\alpha}\right\}_{\alpha \in I}$ is an open covering of $M$ and $g_{\alpha \beta} \in W^{k, p}\left(U_{\alpha \beta}, G\right)(\alpha, \beta \in I)$ satisfy $g_{\alpha \beta}(x) \cdot g_{\beta \gamma}(x)=g_{\alpha \gamma}(x)$ a.e. in $U_{\alpha \beta \gamma}$ whenever $U_{\alpha \beta \gamma} \neq \emptyset$.

(2) $\mathcal{P}_{\text {strong, } G}^{k, p}(M) \subset \mathcal{P}_{G}^{k, p}(M)$ is defined as: $P=\left\langle\left\{U_{\alpha}\right\}_{\alpha \in I},\left\{g_{\alpha \beta}\right\}_{\alpha, \beta \in I}\right\rangle \in \mathcal{P}_{G}^{k, p}(M)$ belongs to $\mathcal{P}_{\text {strong,G }}^{k, p}(M)$ if and only if there exists a sequence of smooth principal $G$-bundles $\left\{P_{n}\right\} \subset \mathcal{P}_{G}^{\infty}(M)\left(\mathcal{P}_{G}^{\infty}(M)\right.$ denotes the set of smooth $G$-bundles on $M)$ of the form $P_{n}=\left\langle\left\{V_{i}\right\}_{i \in J},\left\{g_{i j}^{n}\right\}_{i, j \in J}\right\rangle$ such that $\left\{V_{i}\right\}_{i \in J}$ is a refinement of $\left\{U_{\alpha}\right\}_{\alpha \in I}$, i.e., $V_{i} \subset U_{\varphi(i)}$ for some refinement map $\varphi: J \rightarrow I$ and $\left\|g_{\varphi(i) \varphi(j)}-g_{i j}^{n}\right\|_{W^{k, p}\left(V_{i j}\right)} \rightarrow 0(n \rightarrow \infty)$ for any $i, j \in J$ with $V_{i j} \neq \emptyset$.

(3) $\mathcal{P}_{\text {weak, }, G}^{k, p}(M) \subset \mathcal{P}_{G}^{k, p}(M)$ is defined as: $P=\left\langle\left\{U_{\alpha}\right\}_{\alpha \in I},\left\{g_{\alpha \beta}\right\}_{\alpha, \beta \in I}\right\rangle \in \mathcal{P}_{G}^{k, p}(M)$ belongs to $\mathcal{P}_{\text {weak }, G}^{k, p}(M)$ if and only if there exists a sequence $\left\{P_{n}\right\} \subset \mathcal{P}_{G}^{\infty}(M)$ of the form $P_{n}=\left\langle\left\{V_{i}\right\}_{i \in J},\left\{g_{i j}^{n}\right\}_{i, j \in J}\right\rangle$ such that $\left\{V_{i}\right\}_{i \in J}$ is a refinement of $\left\{U_{\alpha}\right\}_{\alpha \in I}, V_{i} \subset U_{\varphi(i)}$, and $\left\|g_{\varphi(i) \varphi(j)}-g_{i j}^{n}\right\|_{L^{p}\left(V_{i j}\right)} \rightarrow 0(n \rightarrow \infty)$ and $\sup _{n} \sum_{l=1}^{k}\left\|\nabla^{l} g_{i j}^{n}\right\|_{L^{p}\left(V_{i j}\right)}<+\infty$ for any $i, j \in J$ with $V_{i j} \neq \emptyset$, where $\nabla^{l}=\nabla \cdots \nabla$ (l-times).

The isomorphism classes of Sobolev bundles are defined as:

Definition 2.2 We say that $P=\left\langle\left\{U_{\alpha}\right\}_{\alpha \in I},\left\{g_{\alpha \beta}\right\}_{\alpha, \beta \in I}\right\rangle \in \mathcal{P}_{G}^{k, p}(M)$ and $Q=$ $\left\langle\left\{V_{i}\right\}_{i \in J},\left\{h_{i j}\right\}_{i, j \in J}\right\rangle \in \mathcal{P}_{G}^{k, p}(M)$ are $W^{k, p}$-isomorphic to each other if and only if there exist a refinement $\left\{W_{s}\right\}_{s \in K}$ of both of $\left\{U_{\alpha}\right\}$ and $\left\{V_{i}\right\}$ which still covers $M$, i.e., $W_{s} \subset U_{\varphi(s)}, W_{s} \subset V_{\psi(s)}$ for some refinement maps $\varphi: K \rightarrow I, \psi: K \rightarrow J$ and $\bigcup_{s} W_{s}=M$, and $\rho_{s} \in W^{k, p}\left(W_{s}, G\right)(s \in K)$ such that

$$
g_{\varphi(s) \varphi(t)}=\rho_{s} \cdot h_{\psi(s) \psi(t)} \cdot \rho_{t}^{-1} \quad \text { in } W_{s t}
$$

whenever $W_{\text {st }} \neq \emptyset$.

This defines an equivalence relation in $\mathcal{P}_{G}^{k, p}(M)$ and the equivalence class containing $P$ is denoted by $[P]_{k, p}$. The set of $W^{k, p}$-isomorphism classes is denoted by $\hat{\mathcal{P}}_{G}^{k, p}(M)$. In other words, $\hat{\mathcal{P}}_{G}^{k, p}(M)=H^{1}\left(M, \mathcal{W}_{G}^{k, p}\right)$.

The isomorphism classes of $G$-bundles in $\mathcal{P}_{\text {strong, } G}^{k, p}(M)$ and $\mathcal{P}_{\text {weak, } G}^{k, p}(M)$ are defined similarly. However, in these cases there are some choices: One can define $P, Q \in \mathcal{P}_{\text {strong, } G}^{k, p}(M)$ (or $P, Q \in \mathcal{P}_{\text {weak }, G}^{k, p}(M)$ ) are isomorphic to each other if they are isomorphic as bundles in $\mathcal{P}_{G}^{k, p}(M)$ as defined above, i.e., they define the same class in $\hat{\mathcal{P}}_{G}^{k, p}(M)$, or alternately one can define $P$ and $Q$ are 
isomorphic if they define the same class in $H^{1}\left(M, \mathcal{W}_{\mathrm{strong}, G}^{k, p}\right)$ (respectively, $\left.H^{1}\left(M, \mathcal{W}_{\text {weak,G }}^{k, p}\right)\right)$, i.e., in Definition 2.2 , we require $\rho_{p} \in W_{\text {strong }}^{k, p}\left(W_{p}, G\right)$ (respectively, $\left.\rho_{p} \in W_{\text {weak }}^{k, p}\left(W_{p}, G\right)\right)$.

The latter definition seems natural in the sense that if $P=\left\langle\left\{U_{\alpha}\right\},\left\{g_{\alpha \beta}\right\}\right\rangle$ $\in \mathcal{P}_{\text {strong, } G}^{k, p}(M) \quad\left(\right.$ or $\left.P \in \mathcal{P}_{\text {weak }, G}^{k, p}(M)\right)$ and $\rho_{p} \in W_{\text {strong }}^{k, p}\left(W_{p}, G\right)$ (respectively, $\left.\rho_{p} \in W_{\text {weak }}^{k, p}\left(W_{p}, G\right)\right)$, then the gauge transformed bundle $\rho^{*} P:=$ $\left\langle\left\{W_{p}\right\},\left\{\rho_{p} g_{\varphi(p) \varphi(q)} \rho_{q}^{-1}\right\}\right\rangle$ is also in $\mathcal{P}_{\text {strong, } G}^{k, p}(M)$ (respectively, in $\mathcal{P}_{\text {weak, } G}^{k, p}(M)$ ). This is seen from the fact (see below) that $W_{\text {strong }}^{k, p}(U, G)$ and $W_{\text {weak }}^{k, p}(U, G)$ become group under the pointwise multiplication and the inversion for any open subset $U \subset M$.

On the other hand, the first definition is natural in the sense that the invariants defined in the next section for bundles in $\mathcal{P}_{\text {strong, } G}^{k, p}(M)$ or in $\mathcal{P}_{\text {weak,G }}^{k, p}(M)$ are preserved under this equivalence.

Thus when we treat equivalence classes of bundles in $\mathcal{P}_{\text {strong, } G}^{k, p}(M)$ (or in $\left.\mathcal{P}_{\text {weak, }, G}^{k, p}(M)\right)$, we shall indicate which equivalence is used.

Before ending this subsection, we sketch the proof of the assertion that $W_{\text {strong }}^{k, p}(U, G)$ and $W_{\text {weak }}^{k, p}(U, G)$ are groups under the pointwise multiplication and the inversion. (The proof of the same assertion for $W^{k, p}(U, G)$ is given in $[13])$.

Proposition 2.1 For any open set $U \subset M, W_{\text {strong }}^{k, p}(U, G)$ and $W_{\text {weak }}^{k, p}(U, G)$ are groups under the pointwise multiplication and the inversion.

Sketch of the Proof. Let $f, g \in W_{\text {strong }}^{k, p}(U, G)$. There exist $\left\{f_{n}\right\},\left\{g_{n}\right\} \subset$ $C^{\infty}(U, G) \cap W^{k, p}(U, G)$ such that $f_{n} \rightarrow f, g_{n} \rightarrow g$ in $W^{k, p}(U, G)$. From the compactness of $G$, we see that $\left\{f_{n}\right\}$ and $\left\{g_{n}\right\}$ are $L^{\infty}$-bounded and the Gagliardo-Nirenberg inequality [34, III-13-3] implies that $f_{n} g_{n} \rightarrow f g$ in $W^{k, p}(U, G)$, see [13, Appendix] for details. Similarly from the GagliardoNirenberg inequality as in [13, Appendix], we see that $f_{n}^{-1} \rightarrow f^{-1}$ in $W^{k, p}(U, G)$. Thus $f g \in W_{\text {strong }}^{k, p}(U, G)$ and $f^{-1} \in W_{\text {strong }}^{k, p}(U, G)$.

Assume on the other hand $f, g \in W_{\text {weak }}^{k, p}(U, G)$. Then there exist sequences $\left\{f_{n}\right\},\left\{g_{n}\right\} \subset C^{\infty}(U, G) \cap W^{k, p}(U, G)$ such that $f_{n} \rightarrow f$ in $L^{p}(U)$, $\sup _{n} \sum_{j=1}^{k}\left\|\nabla^{j} f_{n}\right\|_{L^{p}(U)}<\infty, g_{n} \rightarrow g$ in $L^{p}(U)$ and $\sup _{n} \sum_{j=1}^{k}\left\|\nabla^{j} g_{n}\right\|_{L^{p}(U)}$ $<\infty$. Then from the compactness of $G$, it is easy to see that $f_{n} g_{n} \rightarrow f g$ in $L^{p}(U)$. Moreover, by the Gagliardo-Nirenberg inequality, we see that $\sup _{n} \sum_{j=1}^{k}\left\|\nabla^{j}\left(f_{n} g_{n}\right)\right\|_{L^{p}(U)}<\infty$. Thus we have $f g \in W_{\text {weak }}^{k, p}(U, G)$. Similarly, we easily see that $f_{n}^{-1} \rightarrow f^{-1}$ in $L^{p}(U)$ and $\sup _{n} \sum_{j=1}^{k}\left\|\nabla^{j}\left(f_{n}^{-1}\right)\right\|_{L^{p}(U)}<\infty$ also by the Gagliardo-Nirenberg inequality. Therefore $f^{-1} \in W_{\text {weak }}^{k, p}(U, G)$ and the proof is complete. 


\subsection{Classes of Sobolev connections}

We introduce in this subsection classes of Sobolev connections on Sobolev bundles introduced in the previous subsection.

Recall that a smooth connection on a smooth principal $G$-bundle $P=$ $\left\langle\left\{U_{\alpha}\right\},\left\{g_{\alpha \beta}\right\}\right\rangle$ is defined by a family of smooth $\mathfrak{g}$-valued 1-forms $\left\{A_{\alpha}\right\}, A_{\alpha} \in$ $C^{\infty}\left(U_{\alpha}, T^{*} U_{\alpha} \otimes \mathfrak{g}\right)$, satisfying the gluing relation

$$
A_{\beta}=g_{\alpha \beta}^{-1} d g_{\alpha \beta}+g_{\alpha \beta}^{-1} A_{\alpha} g_{\alpha \beta} \quad \text { in } U_{\alpha \beta}
$$

whenever $U_{\alpha \beta} \neq \emptyset$.

For a Sobolev bundle as defined in the previous subsection, one may also define a connection on it as a family of $\mathfrak{g}$-valued 1-forms $\left\{A_{\alpha}\right\}_{\alpha \in I}$ belonging to a suitable class of Sobolev space which also satisfies the gluing relation (2.1). In general, a connection loses one more derivatives than the bundle, so one may think that a natural class where $A_{\alpha}$ lives in is the Sobolev class $W^{k-1, p}$. However, this is not so in general. This is because for $g_{\alpha \beta} \in W^{k, p}\left(U_{\alpha \beta}, G\right)$ and $A_{\alpha} \in W^{k-1, p}\left(U_{\alpha}, T^{*} U_{\alpha} \otimes \mathfrak{g}\right)$ the right hand side of (2.1) does not belong to $W^{k-1, p}$ in general: As is shown in the appendix in [13], the Gagliardo-Nirenberg inequality implies $g_{\alpha \beta}^{-1} d g_{\alpha \beta} \in W^{k-1, p}\left(U_{\alpha \beta}\right)$ while the 1 -form $g_{\alpha \beta}^{-1} A_{\alpha} g_{\alpha \beta}$ does not belong to $W^{k-1, p}\left(U_{\alpha \beta}\right)$ for the case $k p<m$ in general. However, if we require some additional regularity for $A_{\alpha}$, we obtain a right definition. Since we are primarily interested in the cases $k=1$ and $k=2$, we only consider these cases. Extensions to the case $k \geq 3$ is straightforward.

Definition 2.3 Assume $k=1$ or $k=2$ and $1 \leq p \leq \infty$. Let $P=$ $\left\langle\left\{U_{\alpha}\right\}_{\alpha \in I},\left\{g_{\alpha \beta}\right\}_{\alpha, \beta \in I}\right\rangle \in \mathcal{P}_{G}^{k, p}(M)$. We define the spaces of Sobolev connections $\mathcal{A}^{k-1, p}(P)$ and $\mathfrak{A}^{1, p / 2}(P)$ on $P$ as follows:

(1) The case $k=1: \mathcal{A}^{0, p}(P)=\mathcal{A}^{p}(P)$ is defined as the set of all $A=$ $\left\{A_{\alpha}\right\}_{\alpha \in I}$ such that $A_{\alpha} \in L^{p}\left(U_{\alpha}, T^{*} U_{\alpha} \otimes \mathfrak{g}\right)$ for all $\alpha \in I$ and (2.1) holds a.e. in $U_{\alpha \beta}$ whenever $U_{\alpha \beta} \neq \emptyset$.

(2) The case $k=2: \mathcal{A}^{1, p}(P)$ is defined as the set of all $A=\left\{A_{\alpha}\right\}_{\alpha \in I}$ such that $A_{\alpha} \in W^{1, p}\left(U_{\alpha}, T^{*} U_{\alpha} \otimes \mathfrak{g}\right) \cap L^{2 p}\left(U_{\alpha}, T^{*} U_{\alpha} \otimes \mathfrak{g}\right)$ for all $\alpha \in I$ and $(2.1)$ holds a.e. in $U_{\alpha \beta}$ whenever $U_{\alpha \beta} \neq \emptyset$.

(3) The case $k=1$ : We define $\mathfrak{A}^{1, p / 2}(P)$ as the set of all $A=\left\{A_{\alpha}\right\}_{\alpha \in I}$ such that $A_{\alpha} \in L^{p}\left(U_{\alpha}, T^{*} U_{\alpha} \otimes \mathfrak{g}\right), d A_{\alpha} \in L^{p / 2}\left(U_{\alpha}, \bigwedge^{2} T^{*} U_{\alpha} \otimes \mathfrak{g}\right)$ and (2.1) holds a.e. in $U_{\alpha \beta}$ whenever $U_{\alpha \beta} \neq \emptyset$.

We must show that the classes $\mathcal{A}^{k, p}(P)(k=0,1)$ and $\mathfrak{A}^{1, p / 2}(k=1)$ are defined properly: For $P \in \mathcal{P}_{G}^{1, p}(M)$ and $A=\left\{A_{\alpha}\right\} \in \mathcal{A}^{p}(P)$, the right hand side of $(2.1)$ belongs to $L^{p}\left(U_{\alpha \beta}\right)$ so $\mathcal{A}^{p}(P)$ is properly defined. For $P \in \mathcal{P}_{G}^{2, p}(M)$ and $A=\left\{A_{\alpha}\right\} \in \mathcal{A}^{1, p}(P)$, the Gagliardo-Nirenberg inequality 
$W^{2, p}\left(U_{\alpha \beta}\right) \cap L^{\infty}\left(U_{\alpha \beta}\right) \subset W^{1,2 p}\left(U_{\alpha \beta}\right)$ implies that the right hand side of (2.1) belongs to $W^{1, p}\left(U_{\alpha \beta}\right)$ and $\mathcal{A}^{1, p}(P)$ is defined properly. Lastly, for $P \in$ $\mathcal{P}_{G}^{1, p}(M)$ and $A=\left\{A_{\alpha}\right\} \in \mathfrak{A}^{1, p / 2}(P)$, the right hand side of (2.1) belongs to $L^{p}\left(U_{\alpha \beta}\right)$ and its exterior derivative

$$
-g_{\alpha \beta}^{-1} d g_{\alpha \beta} g_{\alpha \beta}^{-1} \wedge d g_{\alpha \beta}-g_{\alpha \beta}^{-1} d g_{\alpha \beta} g_{\alpha \beta}^{-1} \wedge A_{\alpha} g_{\alpha \beta}+g_{\alpha \beta}^{-1} d A_{\alpha} g_{\alpha \beta}-g_{\alpha \beta}^{-1} A_{\alpha} \wedge d g_{\alpha \beta}
$$

belongs to $L^{p / 2}\left(U_{\alpha \beta}\right)$ by Hölder's inequality. Thus $\mathcal{A}^{1, p / 2}(P)$ is defined properly.

In applications, more other classes of Sobolev bundles and connections arise naturally. These are considered in $\S 5$.

\section{Topological invariants of Sobolev bundles}

In this section, we define topological invariants for various classes of Sobolev bundles defined in $\S 2$. In [13], we have shown that there is a natural one to one correspondence between $\hat{\mathcal{P}}_{G}^{k, p}(P)$ with $k p=m$ and $\hat{\mathcal{P}}_{G}^{\infty}(M)\left(\hat{\mathcal{P}}_{G}^{\infty}(M)\right.$ is the set of all isomorphism classes of $C^{\infty}$-principal $G$-bundles on $\left.M\right)$. Thus any $W^{k, p}$-bundle with $k p=m$ has a well-defined topology, i.e., it is defined as the corresponding isomorphism class in $\hat{\mathcal{P}}_{G}^{\infty}(M)$. As was shown in [13], this is no longer true for the case $k p<m$. However, for such cases some kinds of topological invariants are well-defined. Giving a definition of invariants and the study of the properties of these are the main purpose of this section.

The invariants are defined for a suitable class of Lipschitz polyhedrons or CW-complexes $K$ and Lipschitz maps $h: K \rightarrow M$. A precise definition of the class of such $K$ is somewhat involved, so we defer it until in the appendix. For example, and this is indeed the main example, any Lipschitz triangulation $h: K \rightarrow M$ of $M$ satisfies our requirement. Thus the reader may assume that $h: K \rightarrow M$ is a Lipschitz triangulation of $M$ throughout this section.

The idea of the definition of the invariants is as follows: Let $P=$ $\left\langle\left\{U_{\alpha}\right\},\left\{g_{\alpha \beta}\right\}\right\rangle \in \mathcal{P}_{G}^{k, p}(M)$ and $h: K \rightarrow M$ be a Lipschitz map (for $K$ as above). We want to consider the pulled back bundle $h^{*} P=\left\langle\left\{h^{-1}\left(U_{\alpha}\right)\right\}\right.$, $\left.\left\{g_{\alpha \beta} \circ h\right\}\right\rangle$. This is not meaningful in general since the function $g_{\alpha \beta} \circ h$ is not well-define for $g_{\alpha \beta} \in W^{k, p}\left(U_{\alpha \beta}, G\right)$ and a Lipschitz map $h: h^{-1}\left(U_{\alpha \beta}\right) \rightarrow M$. However, replacing $h$ by a generic perturbation of $h$, it turns out that it defines a Sobolev bundle on $K$ and has a well-defined topology when $\operatorname{dim} K \leq k p$. When the isomorphism class defined for such a generic perturbation depends only on $h: K \rightarrow M$ and $P$, we can associate a topological invariant for $P \in \mathcal{P}_{G}^{k, p}(M)$ and $h: K \rightarrow M$ as such an isomorphism class. Since the "range" of the dimension $\operatorname{dim} K$ for which the invariants are welldefined depends on the classes $\mathcal{P}_{G}^{k, p}(M), \mathcal{P}_{\text {strong, } G}^{k, p}(M)$ and $\mathcal{P}_{\text {weak, } G}^{k, p}(M)$, we consider each classes separately. 
Before entering into details, we prepare terminologies which will be used throughout this section. Since $M$ is a manifold, it admits a CW-structure. Fix one such structure. Let $e^{i}$ be an $i$-cell of $M$ and $\phi: \mathrm{D}^{i} \rightarrow M$ a characteristic map of $e^{i}$, that is, $\phi: \mathrm{D}^{i} \rightarrow e^{i}$ is surjective, $\phi\left(\partial \mathrm{D}^{i}\right) \subset \partial e^{i}$ and its restriction to the interior of $\mathrm{D}^{i}$ is a homeomorphism onto its image. Here $\mathrm{D}^{i}$ is the unit closed disk in $\mathbb{R}^{i}$. Let $\left\{e_{\alpha}^{i}\right\}$ be the collection of all cells of $M\left(e_{\alpha}^{i}\right.$ denotes one of the $i$-cells of $M)$. For each cell $e_{\alpha}^{i}, \phi_{\alpha}^{i}: \mathrm{D}_{\alpha}^{i} \rightarrow e_{\alpha}^{i}$ denotes the corresponding characteristic map. $\left(\mathrm{D}_{\alpha}^{i}\right.$ is a copy of $\left.\mathrm{D}^{i}\right)$. Set $K=\bigsqcup D_{\alpha}^{i}$. The characteristic maps $\phi_{\alpha}^{i}: \mathrm{D}_{\alpha}^{i} \rightarrow e_{\alpha}^{i}$ induce a map $h=\sqcup \phi_{\alpha}^{i}: K \rightarrow M$ and a homeomorphism $\hat{h}: \hat{K} \rightarrow M$, where $\hat{K}=K / \sim$ and for $x, y \in K$ we define $x \sim y$ if and only if $h(x)=h(y)$. For simplicity, we also call such $h: K \rightarrow M$ or $\hat{h}: \hat{K} \rightarrow M$ a CW-structure of $M$. $\left(M^{i}, h\right)$ denotes the $i$-skeleton of the CW-structure $h: K \rightarrow M$. In this paper, we only consider Lipschitz CW-structures of $M$, i.e., $h: K \rightarrow M$ or $\hat{h}: \hat{K} \rightarrow M$ is Lipschitz. $\mathfrak{C W}(M)$ denotes the set of all Lipschitz CW-structures on $M$. We also use a notation $\{h, K\} \in \mathfrak{C W}(M)$ to denote a CW-structure $h: K \rightarrow M$ $($ or $\hat{h}: \hat{K} \rightarrow M)$.

Next we introduce a genericity notion for $\mathrm{CW}$-structures on $M$. Assume without loss of generality that $M$ is realized as a submanifold of $\mathbb{R}^{l}$ for some large $l>m$. By the tubular neighborhood theorem, there exists $\epsilon>0$ such that the $\epsilon$-neighborhood $\mathcal{O}_{\epsilon}(M)=\left\{\xi \in \mathbb{R}^{l}: d(\xi, M)<\epsilon\right\}$ of $M$ is a smooth fibration $\pi_{M}: \mathcal{O}_{\epsilon}(M) \rightarrow M$, where $\pi_{M}$ is the nearest point projection. Let $\{h, K\} \in \mathfrak{C W}(M)$. Then for any $\xi \in \mathbb{B}_{\epsilon}^{l}=\left\{\xi \in \mathbb{R}^{l}:|\xi|<\epsilon\right\}, h_{\xi}: K \rightarrow M$ defined by $h_{\xi}(x)=\pi_{M}(h(x)+\xi)$ is also a (Lipschitz) CW-structure of $M$. We say that a certain statement holds for generic CW-structures if it holds for all CW-structures of the form $h_{\xi}: K \rightarrow M$, where $\{h, K\} \in \mathfrak{C W}(M)$ and $\xi \in \mathbb{B}_{\epsilon}^{l}$ except for a null set, i.e., there exists a set $N=N(\{h, K\}) \subset \mathbb{B}_{\epsilon}^{l}$ of measure 0 such that for $\xi \in \mathbb{B}_{\epsilon}^{l} \backslash N$ the statement holds.

Finally we give the following remark: From the Gagliardo-Nirenberg inequality $W^{k, p}(U) \cap L^{\infty}(U) \subset W^{1, k p}(U)$, one can obtain a result about Sobolev bundles of the class $W^{k, p}$ from that of the class $W^{1, k p}$, so without loss of generality we restrict our attention to bundles of the class $W^{1, p}$.

\subsection{Topological invariants for $P \in \mathcal{P}_{G}^{k, p}(M)$}

Let $K$ be a polyhedron satisfying the conditions (A-1), (A-2) in the appendix, §7.1. Also let $h: K \rightarrow M$ be a Lipschitz map. For example, a Lipschitz triangulation $h: K \rightarrow M$ satisfies these properties. While $h^{*} P=\left\langle\left\{h^{-1}\left(U_{\alpha}\right)\right\},\left\{g_{\alpha \beta} \circ h\right\}\right\rangle$ does not have a well-defined meaning in general, we shall show that $h_{\xi}^{*} P=\left\langle\left\{h_{\xi}^{-1}\left(U_{\alpha \beta}\right)\right\},\left\{g_{\alpha \beta} \circ h_{\xi}\right\}\right\rangle$ has a well-defined meaning for a.e. $\xi \in \mathbb{B}_{\epsilon}^{l}$. This follows from the following: 
Lemma 3.1 Let $U \subset M$ be an open set and $f \in L^{p}\left(U, \mathbb{R}^{l}\right)\left(\right.$ or $\left.f \in W^{1, p}\left(U, \mathbb{R}^{l}\right)\right)$. Then for a.e. $\xi \in \mathbb{B}_{\epsilon}^{l}, f \circ h_{\xi} \in L^{p}\left(h_{\xi}^{-1}(U), \mathbb{R}^{l}\right.$ ) (respectively, $f \circ h_{\xi} \in$ $\left.W^{1, p}\left(h_{\xi}^{-1}(U), \mathbb{R}^{l}\right)\right)$. Moreover, there exists a constant $C>0$ independent of $f$ such that

$$
\left\|f \circ h_{\xi}\right\|_{L^{p}\left(h_{\xi}^{-1}(U)\right)} \leq C\|f\|_{L^{p}(U)}
$$

(respectively, $\left.\left\|f \circ h_{\xi}\right\|_{W^{1, p}\left(h_{\xi}^{-1}(U)\right)} \leq C\|f\|_{W^{1, p}(U)}\right)$ holds for $\xi \in \mathbb{B}_{\epsilon}^{l}$ of positive measure.

Proof. By Fubini's theorem, we have

$$
\begin{aligned}
\int_{|\xi|<\epsilon} & \left(\int_{h_{\xi}^{-1}(U)}\left|f \circ h_{\xi}(x)\right|^{p} \mathrm{~d} \mathcal{H}^{k}(x)\right) \mathrm{d} \mathcal{H}^{l}(\xi) \\
= & \int_{|\xi|<\epsilon}\left(\int_{K} \chi_{U} \circ h_{\xi}(x)\left|f \circ h_{\xi}(x)\right|^{p} \mathrm{~d} \mathcal{H}^{k}(x)\right) \mathrm{d} \mathcal{H}^{l}(\xi) \\
= & \int_{K}\left(\int_{|\xi|<\epsilon} \chi_{U}\left(\pi_{M}(h(x)+\xi)\right)\left|f\left(\pi_{M}(h(x)+\xi)\right)\right|^{p} \mathrm{~d} \mathcal{H}^{l}(\xi)\right) \mathrm{d} \mathcal{H}^{k}(x) \\
\leq & C \mathcal{H}^{k}(K) \int_{U}|f|^{p} \operatorname{dvol}_{M} .
\end{aligned}
$$

From this, by Fubini's theorem, we have $f \circ h_{\xi} \in L^{p}\left(h_{\xi}^{-1}(U)\right)$ for a.e. $\xi \in \mathbb{B}_{\epsilon}^{l}$. Moreover, there exists $C>0$ (independent of $f$ ) such that for $\xi \in \mathbb{B}_{\epsilon}^{l}$ of positive measure we have

$$
\int_{h_{\xi}^{-1}(U)}\left|f \circ h_{\xi}\right|^{p} \mathrm{~d} \mathcal{H}^{k} \leq C \int_{U}|f|^{p} \operatorname{dvol}_{M} .
$$

In case $f \in W^{1, p}\left(U, \mathbb{R}^{l}\right)$, applying the same argument as above for $d f$, we obtain $d f \circ h_{\xi} \in L^{p}\left(h_{\xi}^{-1}(U)\right)$ for a.e. $\xi \in \mathbb{B}_{\epsilon}^{l}$ and

$$
\int_{h_{\xi}^{-1}(U)}\left|d f \circ h_{\xi}\right|^{p} \mathrm{~d} \mathcal{H}^{k} \leq C \int_{U}|d f|^{p} \operatorname{dvol}_{M}
$$

for some $C>0$ and $\xi \in \mathbb{B}_{\epsilon}^{l}$ of positive measure. Since $d h_{\xi}$ is $L^{\infty}$-bounded and its bound does not depend on $\xi \in \mathbb{B}_{\epsilon}^{l}$, we have $d\left(f \circ h_{\xi}\right) \in L^{p}\left(h_{\xi}^{-1}(U)\right)$ for a.e. $\xi \in \mathbb{B}_{\epsilon}^{l}$ and from (3.2) we obtain

$$
\int_{h_{\xi}^{-1}(U)}\left|d\left(f \circ h_{\xi}\right)\right|^{p} \mathrm{~d} \mathcal{H}^{k} \leq C \int_{U}|d f|^{p} \mathrm{dvol}_{M}
$$

for some another constant $C>0$ and $\xi \in \mathbb{B}_{\epsilon}^{l}$ of positive measure. This completes the proof. 
From Lemma 3.1, we see that for $P=\left\langle\left\{U_{\alpha}\right\},\left\{g_{\alpha \beta}\right\}\right\rangle \in \mathcal{P}_{G}^{1, p}(M)$ and a.e. $\xi \in \mathbb{B}_{\epsilon}^{l}, h_{\xi}^{*} P:=\left\langle\left\{h_{\xi}^{-1}\left(U_{\alpha}\right)\right\},\left\{g_{\alpha \beta} \circ h_{\xi}\right\}\right\rangle \in \mathcal{P}_{G}^{1, p}(K)$. By [13, Corollary 3.1] and its extension to the polyhedron given in the appendix, the $C^{0}$-isomorphism class of $h_{\xi}^{*} P$, denoted by $\left[h_{\xi}^{*} P\right]_{0}$, is well-defined for a.e. $\xi \in \mathbb{B}_{\epsilon}^{l}$ when $\operatorname{dim} K \leq p$. If the class $\left[h_{\xi}^{*} P\right]_{0}$ is independent of a.e. $\xi \in \mathbb{B}_{\epsilon}^{l}$, we will obtain a topological invariant which will depend only on $h: K \rightarrow M$ and $P$. Since the bundle $P$ is not continuous on $M$, the class $\left[h_{\xi}^{*} P\right]_{0}$ may change when it passes through the singularities of $P$ as $\xi$ varies. So in general, we do not expect that $\left[h_{\xi}^{*} P\right]_{0}$ is independent of a.e. $\xi \in \mathbb{B}_{\epsilon}^{l}$. However, if $\operatorname{dim} K$ is slightly small, this is indeed the case.

For $1 \leq p<3$ or $p \geq 4$, define $d(p):=[p-1]$ (the largest integer strictly less than or equal to $p-1)$ and $d(p)=3$ if $3 \leq p<4$. The following is the main result of this subsection.

Theorem 3.1 Let $p \geq 1$ and $P=\left\langle\left\{U_{\alpha}\right\},\left\{g_{\alpha \beta}\right\}\right\rangle \in \mathcal{P}_{G}^{1, p}(M)$. Also let $K$ be a polyhedron satisfying (A-1), (A-2) in the appendix and $h: K \rightarrow M$ a Lipschitz map. Assume that $\operatorname{dim} K \leq d(p)$. Then the class $\left[h_{\xi}^{*} P\right]_{0}$ defined for a.e. $\xi \in \mathbb{B}_{\epsilon / 3}^{l}$ is independent of a.e. $\xi \in \mathbb{B}_{\epsilon / 3}^{l}$. Moreover, the class $\left[h_{\xi}^{*} P\right]_{0}$ depends only on the homotopy class of $h: K \rightarrow M$ and the $W^{1, p}$-isomorphism class of $P$. In other words, there exists a homomorphism $h^{*}: \check{H}^{1}\left(M ; \mathcal{W}_{G}^{1, p}\right) \rightarrow \check{H}^{1}\left(K ; \mathfrak{C}_{G}^{0}\right)$ which depends only on the homotopy class $[h] \in[K, M]$ and is defined by $h^{*}[P]_{1, p}=\left[h_{\xi}^{*} P\right]_{0}$ for a.e. $\xi \in \mathbb{B}_{\epsilon / 3}^{l}$, where $[P]_{1, p}$ is the class of $P$ in $\hat{\mathcal{P}}_{G}^{1, p}(M)=\check{H}^{1}\left(M ; \mathcal{W}_{G}^{1, p}\right)$.

Remark 3.1 The above result is analogous to the corresponding result of Sobolev maps as developed by [42], see also [10]. The $([p]-1)$-homotopy class of a Sobolev map in $W^{1, p}(M, N)$ (for $M, N$ compact manifolds) is welldefined and this is in general optimal, i.e., maps in $W^{1, p}(M, N)$ do not have a well-defined [p]-homotopy type in general, see [42]. In view of this, one may think that the above result is also optimal. Indeed, if we slightly relax the definition of Sobolev bundles, we have the same result as stated in Theorem 3.1 and the result is optimal for such classes of Sobolev bundles, see 55 . However, we do not know the above result is optimal for $P \in \mathcal{P}_{G}^{1, p}(M)$. Indeed, some topological invariants associated with $P \in \mathcal{P}_{G}^{1, p}(M)$ are welldefined beyond the dimension $d(p)$, see $\S 4$, so one may suspect that the above result also holds up to $\operatorname{dim} K=[p]$. The improvement for the case $3 \leq p<4$ is due to the fact that $\pi_{2}(G)=0$ for any compact Lie group $G$.

The proof of Theorem 3.1 is decomposed into several steps. We first show the independence of the isomorphism classes $\left[h_{\xi} P\right]_{0}$ for a.e. $\xi \in \mathbb{B}_{\epsilon / 3}^{l}$ when $\operatorname{dim} K \leq[p]-1$. Improvement for the case $3 \leq p<4$ will be given latter. In the following, the set of all isomorphism classes of $C^{0}$-principal $G$-bundles on $K$ will be denoted by $\hat{\mathcal{P}}_{G}^{0}(K)$. 
Lemma 3.2 Let $P=\left\langle\left\{U_{\alpha}\right\},\left\{g_{\alpha \beta}\right\}\right\rangle \in \mathcal{P}_{G}^{1, p}(M)$ and $K$ a polyhedron satisfying (A-1), (A-2) in the appendix and $\operatorname{dim} K \leq[p]-1$. Also let $h: K \rightarrow M$ be a Lipschitz map. Then the class $\left[h_{\xi}^{*} P\right]_{0} \in \hat{\mathcal{P}}_{G}^{0}(K)$ is independent of a.e. $\xi \in \mathbb{B}_{\epsilon / 3}^{l}$.

Proof. Let $\xi \in \mathbb{B}_{2 \epsilon / 3}^{l}$. Then $h$ is homotopic to $h_{\xi}$ by the homotopy $[0,1] \times$ $K \ni(x, t) \mapsto \pi_{M}(h(x)+t \xi) \in M$. For the purpose of our argument, we use the following another homotopy: Let $\varphi \in C^{\infty}(\mathbb{R})$ be such that $\varphi(t)=0$ for $t \leq 0, \varphi(t)=1$ for $1 \leq t$ and $0 \leq \varphi(t) \leq 1$ for $0<t<1$. Define $H_{\xi}: \mathbb{R} \times K \rightarrow M$ by $H_{\xi}(t, x)=\pi_{M}(h(x)+\varphi(t) \xi)$. $H_{\xi}$ satisfies $H_{\xi}(t, x)=h(x)$ for $t \leq 0, H_{\xi}(t, x)=h_{\xi}(x)$ for $1 \leq t$ and gives a homotopy between $h$ and $h_{\xi}$.

For $\xi \in \mathbb{B}_{2 \epsilon / 3}^{l}, \zeta \in \mathbb{B}_{\epsilon / 3}^{l}$, define $H_{\xi, \zeta}: \mathbb{R} \times K \rightarrow M$ by $H_{\xi, \zeta}(x, y)=$ $\pi_{M}(h(x)+\varphi(t) \xi+\zeta)$. For an arbitrary fixed $\xi \in \mathbb{B}_{2 \epsilon / 3}^{l}$ and a compact interval $I \subset \mathbb{R}$, by Lemma $3.1 H_{\xi, \zeta}^{*} P=\left\langle\left\{H_{\xi, \zeta}^{-1}\left(U_{\alpha}\right)\right\},\left\{g_{\alpha \beta} \circ H_{\xi, \zeta}\right\}\right\rangle$ restricted to $I \times K$ is a Sobolev bundle of class $W^{1, p}$ on $I \times K$ for a.e. $\zeta \in \mathbb{B}_{\epsilon / 3}^{l}$. In particular, this holds for $I=[-1,2]$ and we denote the corresponding Sobolev bundle on $[-1,2] \times K$ by the same symbol $H_{\xi, \zeta}^{*} P$. Notice that when $\operatorname{dim} K \leq[p]-1$, the class $\left[H_{\xi, \zeta}^{*} P\right]_{0} \in \hat{\mathcal{P}}_{G}^{0}([-1,2] \times K)$ is well-defined for a.e. $\zeta \in \mathbb{B}_{\epsilon / 3}^{l}$ since $\operatorname{dim}([-1,2] \times K) \leq p$. To see this, one needs to check the conditions (A-1) and (A-2) in the appendix. Let $\pi_{K}: \mathcal{O}(K) \rightarrow K$ be as in the appendix. Then id $\times \pi_{K}: \mathbb{R} \times \mathcal{O}(K) \rightarrow \mathbb{R} \times K$ satisfies the conditions (A-1) and (A-2) for $l$ replaced by $l+1$ and $m$ replaced by $m+1$, where id is the identity map id $: \mathbb{R} \rightarrow \mathbb{R}$. Then arguing as in the appendix, we see that there exists a well-defined associated topological class for $H_{\xi, \zeta}^{*} P \in \mathcal{P}_{G}^{1, p}([-1,2] \times K)$ for a.e. $\zeta \in \mathbb{B}_{\epsilon / 3}^{l}$.

Let us recall how the $C^{0}$-isomorphism class is associated to $H_{\xi, \zeta}^{*} P$. We may assume that, passing to a finite refinement if necessary, $\left\{U_{\alpha}\right\}$ is a finite cover of $M$. Then by the approximation theorem [13] (see also the appendix), there exists a refinement $\left\{V_{\alpha}\right\}$ of $\left\{H_{\xi, \zeta}^{-1}\left(U_{\alpha}\right)\right\}$ such that $\bigcup_{\alpha} V_{\alpha}=[-1,2] \times M$ and $V_{\alpha} \subset H_{\xi, \zeta}^{-1}\left(U_{\alpha}\right)$ and there exist principal $G$-bundles $P_{\epsilon}=\left\langle\left\{V_{\alpha}\right\},\left\{g_{\alpha \beta}^{\epsilon}\right\}\right\rangle$ on $[-1,2] \times K$ of Lipschitz class such that

$$
\left\|g_{\alpha \beta} \circ H_{\xi, \zeta}-g_{\alpha \beta}^{\epsilon}\right\|_{W^{1, p}\left(V_{\alpha \beta}\right)} \rightarrow 0 \quad \text { as } \epsilon \rightarrow 0 .
$$

As proved in [13] (see also the appendix), the $C^{0}$-isomorphism class of $P_{\epsilon}$ is uniquely determined by $H_{\xi, \zeta}^{*} P$ for small $\epsilon>0$ and this class is denoted by $\left[H_{\xi, \zeta}^{*} P\right]_{0} \in \hat{\mathcal{P}}_{G}^{0}([-1,2] \times K)$. In the above case, since $H_{\xi, \zeta}^{-1}\left(U_{\alpha}\right) \cap([-1,0] \times M)$ and $H_{\xi, \zeta}^{-1}\left(U_{\alpha}\right) \cap([1,2] \times M)$ are written as products $[-1,0] \times h_{\zeta}^{-1}\left(U_{\alpha}\right)$ and $[1,2] \times h_{\xi+\zeta}^{-1}\left(U_{\alpha}\right)$ respectively, we may assume that $V_{\alpha} \cap\left(I_{i} \times M\right)(i=0,1)$ is also product for some interval $I_{0} \subset[-1,0]$ and $I_{1} \subset[1,2]$. 
By Lemma 3.1, for a.e. $\zeta \in \mathbb{B}_{\epsilon / 3}^{l},\left.H_{\xi, \zeta}^{*} P\right|_{\{0\} \times K}=h_{\zeta}^{*} P=\left\langle\left\{V_{\alpha}^{t_{0}}\right\},\left\{g_{\alpha \beta} \circ h_{\zeta}\right\}\right\rangle$ and $\left.H_{\xi, \zeta}^{*} P\right|_{\{1\} \times K}=h_{\xi+\zeta}^{*} P=\left\langle\left\{V_{\alpha}^{t_{1}}\right\},\left\{g_{\alpha \beta} \circ h_{\xi+\zeta}\right\}\right\rangle$ are Sobolev bundles of class $W^{1, p}$, where $t_{0} \in I_{0}, t_{1} \in I_{1}$ and for $t \in[-1,2], V_{\alpha}^{t}:=(\{t\} \times K) \cap V_{\alpha}$ is the $t$-slice of $V_{\alpha}$. Since $\operatorname{dim} K \leq[p]-1$, by the Sobolev embedding $W^{1, p} \subset C^{0}$ in dimension less than $p$, these bundles are of class $C^{0}$.

We claim that for fixed $\xi \in \mathbb{B}_{2 \epsilon / 3}^{l}, h_{\zeta}^{*} P$ is $C^{0}$-isomorphic to $h_{\xi+\zeta}^{*} P$ for a.e. $\zeta \in \mathbb{B}_{\epsilon / 3}^{l}$. To prove the claim, we first observe that from (3.4), the Fubini's theorem and the Fatou's lemma, there exists a sequence $\left\{\epsilon_{k}\right\}$ converging to $0, t_{0} \in I_{0}$ and $t_{1} \in I_{1}$ such that

$$
\begin{gathered}
\left\|g_{\alpha \beta} \circ H_{\xi, \zeta}\left(t_{0}, \cdot\right)-g_{\alpha \beta}^{\epsilon_{k}}\left(t_{0}, \cdot\right)\right\|_{W^{1, p}\left(V_{\alpha \beta}^{t_{0}}\right)} \rightarrow 0, \\
\left\|g_{\alpha \beta} \circ H_{\xi, \zeta}\left(t_{1}, \cdot\right)-g_{\alpha \beta}^{\epsilon_{k}}\left(t_{1}, \cdot\right)\right\|_{W^{1, p}\left(V_{\alpha \beta}^{t_{1}}\right)} \rightarrow 0
\end{gathered}
$$

as $k \rightarrow \infty$, where $V_{\alpha \beta}^{t}=V_{\alpha}^{t} \cap V_{\beta}^{t}$.

Since $H_{\xi, \zeta}\left(t_{0}, \cdot\right)=h_{\zeta}, H_{\xi, \zeta}\left(t_{1}, \cdot\right)=h_{\xi+\zeta}$ and the Sobolev embedding $W^{1, p} \subset C^{0}$ in dimension less than $p$, we obtain from (3.5) and (3.6)

$$
\begin{array}{r}
\left\|g_{\alpha \beta} \circ h_{\zeta}-g_{\alpha \beta}^{\epsilon_{k}}\left(t_{0}, \cdot\right)\right\|_{C^{0}\left(V_{\alpha \beta}^{t_{0}}\right)} \rightarrow 0, \\
\left\|g_{\alpha \beta} \circ h_{\xi+\zeta}-g_{\alpha \beta}^{\epsilon_{k}}\left(t_{1}, \cdot\right)\right\|_{C^{0}\left(V_{\alpha \beta}^{t_{1}}\right)} \rightarrow 0
\end{array}
$$

as $k \rightarrow \infty$.

Since $\left\{V_{\alpha}^{t_{0}}\right\}$ and $\left\{V_{\alpha}^{t_{1}}\right\}$ are refinements of the open covers $\left\{h_{\zeta}^{-1}\left(U_{\alpha}\right)\right\}$ and $\left\{h_{\xi+\zeta}^{-1}\left(U_{\alpha}\right)\right\}$ respectively, from (3.7) and (3.8) we see that the $C^{0}$-bundles $h_{\zeta}^{*} P$ and $h_{\xi+\zeta}^{*} P$ are $C^{0}$-isomorphic to $\left.P_{\epsilon_{k}}\right|_{\left\{t_{0}\right\} \times K}$ and $\left.P_{\epsilon_{k}}\right|_{\left\{t_{1}\right\} \times K}$ respectively for large $k$. Since $P_{\epsilon_{k}}$ is a bundle over $[-1,2] \times K,\left.P_{\epsilon_{k}}\right|_{\left\{t_{0}\right\} \times K}$ is isomorphic to $\left.P^{\epsilon_{k}}\right|_{\left\{t_{1}\right\} \times K}$. Thus as principal $G$-bundles of class $C^{0}, h_{\xi+\zeta}^{*} P$ is isomorphic to $h_{\zeta}^{*} P$. Since this holds for a.e. $\zeta \in \mathbb{B}_{\epsilon / 3}^{l}$, the claim is proved.

From this the lemma follows as follows. Let $E_{0} \subset \mathbb{B}_{\epsilon / 3}^{l}$ be a measure zero set of $\xi$ on which $h_{\xi}^{*} P$ is not defined. Assume otherwise that $\left[h_{\xi}^{*} P\right]_{0}$ is not constant for a.e. $\xi \in B_{\epsilon / 3} \backslash E_{0}$. We first notice that $\hat{\mathcal{P}}_{G}^{0}(K)$ is a countable set. This is because the set of homotopy classes $[K, B G]$ ( $B G$ is the classifying space for $G$-bundles or a finite dimensional approximation of it) is a countable set for compact $K$. Thus under the assumption, we may find two different isomorphism classes $\left[P_{1}\right]_{0},\left[P_{2}\right]_{0} \in \hat{\mathcal{P}}_{G}^{0}(K)$ such that $\left[h_{\xi}^{*} P\right]_{0}=\left[P_{1}\right]_{0}$ for $\xi \in E_{1} \subset \mathbb{B}_{\epsilon / 3}^{l}$ with $\mathcal{H}^{m}\left(E_{1}\right)>0$ and $\left[h_{\xi}^{*} P\right]_{0}=\left[P_{2}\right]_{0}$ for $\xi \in E_{2} \subset \mathbb{B}_{\epsilon / 3}^{l}$ with $\mathcal{H}^{m}\left(E_{2}\right)>0$. Let $\xi_{i} \in E_{i}(i=1,2)$ be a density point, i.e.,

$$
\lim _{r \downarrow 0} \frac{\mathcal{H}^{m}\left(B_{r}\left(\xi_{i}\right) \cap E_{i}\right)}{\mathcal{H}^{m}\left(B_{r}\left(\xi_{i}\right)\right)}=1 .
$$


Let $\xi=\xi_{1}-\xi_{2} \in \mathbb{B}_{2 \epsilon / 3}^{l}$. Then from what we have proved above, we obtain $\left[h_{\xi+\zeta}^{*} P\right]_{0}=\left[h_{\zeta}^{*} P\right]_{0}$ for $\zeta \in \mathbb{B}_{\epsilon / 3}^{l} \backslash\left(E_{\xi} \cup E_{0} \cup\left(E_{0}-\xi\right)\right)$, where $E_{\xi} \subset B_{\epsilon / 3}$ is a measure zero set such that $\left[h_{\xi+\zeta}^{*} P\right]_{0}=\left[h_{\zeta}^{*} P\right]_{0}$ for $\zeta \in B_{\epsilon / 3} \backslash E_{\xi}$. Since $\xi+\xi_{2}=\xi_{1}$ is a density point of $E_{2}+\xi, \xi_{1}$ is a density point for both $E_{1}$ and $\left(E_{2} \backslash\left(E_{\xi} \cup E_{0} \cup\left(E_{0}-\xi\right)\right)+\xi\right.$. From this, we have in particular $E_{1} \cap\left(\left(E_{2} \backslash\right.\right.$ $\left.\left(E_{\xi} \cup E_{0} \cup\left(E_{0}-\xi\right)\right)+\xi\right) \neq \emptyset$. Choose $\xi_{1}^{\prime} \in E_{1}, \xi_{2}^{\prime} \in E_{2} \backslash\left(E_{\xi} \cup E_{0} \cup\left(E_{0}-\xi\right)\right)$ such that $\xi_{1}^{\prime}=\xi+\xi_{2}^{\prime}$. Then we have $\left[P_{1}\right]_{0}=\left[h_{\xi_{1}^{\prime}}^{*} P\right]_{0}=\left[h_{\xi_{2}^{\prime}}^{*} P\right]_{0}=\left[P_{2}\right]_{0}$, a contradiction. This completes the proof.

Definition 3.1 Let $P=\left\langle\left\{U_{\alpha}\right\},\left\{g_{\alpha \beta}\right\}\right\rangle \in \mathcal{P}_{G}^{1, p}(M), K$ a polyhedron satisfying (A-1), (A-2) in the appendix, $\operatorname{dim} K \leq[p]-1$ and $h: K \rightarrow M a$ Lipschitz map. We denote by $[P]_{h, K} \in \hat{\mathcal{P}}_{G}^{0}(K)$ the class $\left[h_{\xi}^{*} P\right]_{0} \in \hat{\mathcal{P}}_{G}^{0}(K)$ which is independent of a.e. $\xi \in \mathbb{B}_{\epsilon / 3}^{l}$.

We next show that the class $[P]_{h, K}$ is invariant under $W^{1, p}$-bundle isomorphisms. That is, we have:

Lemma 3.3 Let $P=\left\langle\left\{U_{\alpha}\right\},\left\{g_{\alpha \beta}\right\}\right\rangle, Q=\left\langle\left\{V_{i}\right\},\left\{h_{i j}\right\}\right\rangle \in \mathcal{P}_{G}^{1, p}(M), K a$ polyhedron satisfying (A-1), (A-2) in the appendix, $\operatorname{dim} K \leq[p]-1$ and $h: K \rightarrow M$ a Lipschitz map. Assume $P$ is $W^{1, p}$-equivalent to $Q$. Then we have $[P]_{h, K}=[Q]_{h, K}$.

Proof. By the assumption, there exist a refinement $\left\{W_{s}\right\}$ of $\left\{U_{\alpha}\right\}$ and $\left\{V_{i}\right.$, i.e., $\bigcup_{s} W_{s}=M, W_{s} \subset V_{\varphi(s)}$ and $W_{s} \subset V_{\psi(s)}$ and $\rho=\left\{\rho_{s}\right\}$ such that $\rho_{s} \in W^{1, p}\left(W_{s}, G\right)$ and

$$
g_{\varphi(s) \varphi(t)}=\rho_{s} h_{\psi(s) \psi(t)} \rho_{t}^{-1}
$$

on $W_{s t}:=W_{s} \cap W_{t}$.

On the other hand, by Lemma 3.1 and the Sobolev embedding we have $h_{\xi}^{*} P, h_{\xi}^{*} Q \in \mathcal{P}_{G}^{0}(K)$ and $\rho_{s} \circ h_{\xi} \in C^{0}\left(h_{\xi}^{-1}\left(W_{s}\right), G\right)$ for a.e. $\xi \in \mathbb{B}_{\epsilon}$ and from (3.9) we obtain

$$
g_{\varphi(s) \varphi(t)} \circ h_{\xi}=\left(\rho_{s} \circ h_{\xi}\right)\left(h_{\psi(s) \psi(t)} \circ h_{\xi}\right)\left(\rho_{t} \circ h_{\xi}\right)^{-1}
$$

on $h_{\xi}^{-1}\left(W_{s t}\right)$.

From (3.10), we conclude that $h_{\xi}^{*} P$ is $C^{0}$-isomorphic to $h_{\xi}^{*} Q$ for a.e. $\xi \in \mathbb{B}_{\epsilon}^{l}$. Then by the definition of the classes $[P]_{h, K}$ and $[Q]_{h, K}$ given above, we have $[P]_{h, K}=[Q]_{h, K}$.

The final ingredient to complete the proof of Theorem 3.1 is:

Lemma 3.4 Let $P=\left\langle\left\{U_{\alpha}\right\},\left\{g_{\alpha \beta}\right\}\right\rangle \in \mathcal{P}_{G}^{1, p}(M), K$ a polyhedron satisfying (A-1), (A-2) in the appendix, $\operatorname{dim} K \leq[p]-1$ and $h, h^{\prime}: K \rightarrow M$ Lipschitz maps. Suppose that $h$ is homotopic to $h^{\prime}$, then we have $[P]_{h, K}=[P]_{h^{\prime}, K}$. 
Proof. Let $H: \mathbb{R} \times K$ be a Lipschitz homotopy between $h$ and $h^{\prime}$ such that $H(t, \cdot)=h$ for $t \leq 0$ and $H(t, \cdot)=h^{\prime}$ for $t \geq 1$. For a.e. $\xi \in \mathbb{B}_{\epsilon}^{l}$, define $H_{\xi}(t, x)=\pi_{M}(H(t, x)+\xi)$. Then by Lemma 3.1, for a.e. $\xi \in \mathbb{B}_{\epsilon}^{l}$ $H_{\xi}^{*} P=\left\langle\left\{H_{\xi}^{-1}\left(U_{\alpha}\right)\right\},\left\{g_{\alpha \beta} \circ H_{\xi}\right\}\right\rangle$ defines a principal $G$-bundle of class $W^{1, p}$ on $[-1,2] \times K$. Then by the same argument as in the proof of Lemma 3.2, we see that for a.e. $\xi \in \mathbb{B}_{\epsilon}^{l}$ and some $t_{0} \in[-1,0]$ and $t_{1} \in[1,2],\left.H_{\xi}^{*} P\right|_{\left\{t_{0}\right\} \times K}$ and $\left.H_{\xi}^{*} P\right|_{\left\{t_{1}\right\} \times K}$ define principal $G$-bundles of class $W^{1, p}$ and they are isomorphic as $C^{0}$-bundles to each other; $\left.\left.H_{\xi}^{*} P\right|_{\left\{t_{0}\right\} \times K} \cong H_{\xi}^{*} P\right|_{\left\{t_{1}\right\} \times K}$. Since $h_{\xi}^{*} P=$ $\left.H_{\xi}^{*} P\right|_{\left\{t_{0}\right\} \times K}$ and $\left(h_{\xi}^{\prime}\right)^{*} P=\left.H_{\xi}^{*} P\right|_{\left\{t_{1}\right\} \times K}$, we conclude that $h_{\xi} P \cong\left(h_{\xi}^{\prime}\right)^{*} P$ for a.e. $\xi \in \mathbb{B}_{\epsilon}^{l}$. Thus by the definition, we have $[P]_{h, K}=[P]_{h^{\prime}, K}$.

For the case $3 \leq p<4$, the above results can be improved. That is, we have:

Lemma 3.5 Assume $3 \leq p<4$. Let $P=\left\langle\left\{U_{\alpha}\right\},\left\{g_{\alpha \beta}\right\}\right\rangle \in \mathcal{P}_{G}^{1, p}(M)$ and $K$ a polyhedron satisfying (A-1), (A-2) in the appendix and $\operatorname{dim} K \leq 3$. Also let $h: K \rightarrow M$ be a Lipschitz map. Then the class $\left[h_{\xi}^{*} P\right]_{0} \in \mathcal{P}_{G}^{0}(K)$ is independent of a.e. $\xi \in \mathbb{B}^{l} \epsilon / 3$.

Proof: We have already shown the result for the case $\operatorname{dim} K \leq[p]-1=2$. Thus we assume $\operatorname{dim} K=3$. We denote by $K^{2}$ the 2 -skeleton of $K$. As we have shown, there exists a well-defined class $\left[h_{\xi}^{*}\right] P_{0} \in \mathcal{P}_{G}^{0}(K)$ for a.e. $\xi \in \mathbb{B}_{\epsilon}^{l}$ and its restriction to $K^{2},\left[\left(\left.h_{\xi}\right|_{K^{2}}\right)^{*} P\right]_{0} \in \mathcal{P}_{G}^{0}\left(K^{2}\right)$ is independent of a.e. $\xi \in \mathbb{B}_{\epsilon / 3}^{l}$. We need to show that $\left[h_{\xi}^{*} P\right]_{0} \in \mathcal{P}_{G}^{0}(K)$ is also independent of a.e. $\xi \in \mathbb{B}_{\epsilon}^{l}$. Thus the result follows from the following lemma applied to $P_{1}=h_{\xi}^{*} P$ and $P_{2}=h_{\eta}^{*} P$ for two different a.e. $\xi, \eta \in \mathbb{B}_{\epsilon}^{l}$.

Lemma 3.6 Let $K$ be a 3-dimensional polyhedron and $P_{1}, P_{2} \rightarrow K$ two principal $G$-bundles which satisfy $\left.\left.P_{1}\right|_{K^{2}} \cong P_{2}\right|_{K^{2}}$. Then $P_{1} \cong P_{2}$ over $K$.

Proof. Since $\pi_{2}(G)=0$ for any compact Lie group $G$, this is an easy consequence of the obstruction theory. For completeness, we give a proof.

Let $E B \rightarrow B G$ be the universal $G$-bundle, i.e., a $G$-bundle whose total space $E G$ is contractible, see [12]. From the bundle classification theory [12], any principal $G$-bundle $P \rightarrow K$ is obtained as a pull back of $E G \rightarrow B G$ via a map $f_{P}: K \rightarrow B G$, i.e., $P \cong f_{P}^{*} E G$. Moreover, this gives a bijective correspondence between the homotopy classes $[K, M]$ and $\hat{\mathcal{P}}_{G}^{0}(K)$. Thus for $P_{1}, P_{2}$ as above, there exist continuous $f_{1}, f_{2}: K \rightarrow B G$ such that $P_{1} \cong f_{1}^{*} E G$ and $P_{2} \cong f_{2}^{*} E G$. By our assumption, $f_{1}$ is homotopic to $f_{2}$ when restricted to $K^{2}$, i.e., there exists a homotopy $F:[0,1] \times K^{2} \rightarrow M$ such that $F(0, \cdot)=f_{1}$ and $F(1, \cdot)=f_{2}$. To prove $P_{1} \cong P_{2}$, it suffices to show that $F$ has a continuous extension $\tilde{F}$ to $[0,1] \times K$ such that $\tilde{F}(0, x)=f_{1}(x)$ and 
$\tilde{F}(1, x)=f_{2}(x)$ for $x \in K$. By the obstruction theory [5], the obstruction to extending $F$ to such $\tilde{F}$ lies in $H^{i+1}\left([0,1] \times K,[0,1] \times K^{2} \cup\{0\} \times K \cup\{1\} \times\right.$ $\left.K ; \pi_{i}(B G)\right) \cong H^{i}\left(K, K^{2} ; \pi_{i}(B G)\right)$ for $i \geq 0$. The latter cohomology groups are all trivial since trivially we have $H^{i}\left(K, K^{2} ; \pi_{i}(B G)\right)=0$ for $i=1,2$ and $i>3$ and $H^{3}\left(K, K^{2} ; \pi_{3}(B G)\right)=H^{3}\left(K, K^{2} ; \pi_{2}(G)\right)=0$ since $\pi_{2}(G)=0$ for a compact Lie group $G$.

Let $P, K$ and $h$ be as in Lemma 3.5. As in Definition 3.1, from Lemma 3.5 we denote by $[P]_{h, K} \in \hat{\mathcal{P}}_{G}^{0}(K)$ the class $\left[h_{\xi}^{*} P\right]_{0} \in \hat{\mathcal{P}}_{G}^{0}(K)$ which is independent of a.e. $\xi \in \mathbb{B}_{\epsilon / 3}^{l}$. As a corollary of Lemma 3.6, we have

Lemma 3.7 Assume $3 \leq p<4$. Let $K$ be a polyhedron satisfying (A-1) and (A-2) in the appendix, $\operatorname{dim} K=3$ and $h, h^{\prime}: K \rightarrow M$ Lipschitz maps. Assume $h$ is homotopic to $h^{\prime}$. Then we have $[P]_{h, K}=[P]_{h^{\prime}, K}$.

Proof. As we have shown in Lemma 3.4, for a.e. $\xi \in \mathbb{B}_{\epsilon / 3}^{l}$ we have $h_{\xi}^{*} P \cong$ $h_{\xi}^{\prime *} P$ over $K^{2}$. Thus by Lemma 3.6, we obtain $h_{\xi}^{*} P \cong h_{\xi}^{\prime *} P$ over $K$ for such $\xi \in \mathbb{B}_{\epsilon}^{l}$. From this, the result follows.

From Lemma 3.4 and Lemma 3.7, we give the following definition:

Definition 3.2 Assume $p \geq 1$. Let $P=\left\langle\left\{U_{\alpha}\right\},\left\{g_{\alpha \beta}\right\}\right\rangle \in \mathcal{P}_{G}^{1, p}(M), K$ a polyhedron satisfying (A-1), (A-2) in the appendix, $\operatorname{dim} K \leq d(p)$ and $h: K \rightarrow M$ a Lipschitz map. We denote by $[P]_{[h], K} \in \hat{\mathcal{P}}_{G}^{0}(K)$ the class $\left[h_{\xi}^{*} P\right]_{0} \in \hat{\mathcal{P}}_{G}^{0}(K)$ which is independent of a.e. $\xi \in \mathbb{B}_{\epsilon / 3}^{l}$ and depends only on the homotopy class of $h$.

From Definition 3.2, we obtain various invariants of $P \in \mathcal{P}_{G}^{1, p}(M)$ for varying $K$ and $h$. Among them $[P]_{[h], K^{d(p)}}$ for $h: K^{d(p)} \rightarrow M$, where $h: K \rightarrow M$ is a CW-structure of $M$, is special in the sense that they determine all the other invariants. That is, we have:

Lemma 3.8 Assume $p \geq 1$. Let $P_{1}, P_{2} \in \mathcal{P}_{G}^{1, p}(M)$ and $h: K \rightarrow M a$ $C W$-structure of $M$, i.e., $\{h, K\} \in \mathfrak{C W}(M)$. Assume that $\left[P_{1}\right]_{[h], K^{d(p)}}=$ $\left[P_{1}\right]_{[h], K^{d(p)}}$. Then for any polyhedron $L$ satisfying $(\mathrm{A}-1)$ and $(\mathrm{A}-2)$ in the appendix, $\operatorname{dim} L \leq d(p)$ and any Lipschitz $\operatorname{map} \varphi: L \rightarrow M$, we have $\left[P_{1}\right]_{[\varphi], L}=\left[P_{2}\right]_{[\varphi], L}$.

Proof. We first remark that for $P=\left\langle\left\{U_{\alpha}\right\},\left\{g_{\alpha \beta}\right\}\right\rangle \in \mathcal{P}_{G}^{1, p}(M)$ and $\left\{V_{i}\right\}$ a refinement of $\left\{U_{\alpha}\right\}$, i.e., $\bigcup_{i} V_{i}=M$ and $V_{i} \subset U_{\psi(i)}$ for some refinement map $\psi$, the bundle $P^{\prime}=\left\langle\left\{V_{i}\right\},\left\{g_{\psi(i) \psi(j)}\right\}\right\rangle \in \mathcal{P}_{G}^{1, p}(M)$ has the same invariant $[P]_{[h], K}=\left[P^{\prime}\right]_{[h], K}$ for $K$ and $h$ as in Definition 3.2. To see this, recall that $[P]_{[h], K}$ and $\left[P^{\prime}\right]_{[h], K}$ are defined as the classes of $h_{\xi}^{*} P=\left\langle\left\{h_{\xi}^{-1}\left(U_{\alpha}\right)\right\},\left\{g_{\alpha \beta} \circ\right.\right.$ $\left.\left.h_{\xi}\right\}\right\rangle \in \mathcal{P}_{G}^{0}(K)$ and $h_{\xi}^{*} P^{\prime}=\left\langle\left\{h_{\xi}^{-1}\left(V_{i}\right)\right\},\left\{g_{\psi(i) \psi(j)} \circ h_{\xi}\right\}\right\rangle \in \mathcal{P}_{G}^{0}(K)$, respectively, 
for a.e. $\xi \in \mathbb{B}_{\epsilon / 3}^{l}$ (for $p=3$, they are defined as the $C^{0}$-isomorphism classes associated to $h_{\xi}^{*} P, h_{\xi}^{*} P^{\prime} \in \mathcal{P}_{G}^{1,3}\left(M^{3}\right)$. Because $\left\{h_{\xi}^{-1}\left(V_{i}\right)\right\}$ is a refinement of $\left\{h_{\xi}^{-1}\left(U_{\alpha}\right)\right\}$, they are isomorphic and the claim follows.

By the above observation, passing to a refinement if necessary, we may assume that $P_{1}$ and $P_{2}$ are of the form $P_{1}=\left\langle\left\{U_{\alpha}\right\},\left\{g_{\alpha \beta}^{1}\right\}\right\rangle, P_{2}=\left\langle\left\{U_{\alpha}\right\},\left\{g_{\alpha \beta}^{2}\right\}\right\rangle$. By definition, $\left[P_{1}\right]_{[h], K^{d(p)}}$ and $\left[P_{2}\right]_{[h], K^{d(p)}}$ are given as

$$
\left[P_{1}\right]_{[h], K^{d(p)}}=\left[h_{\xi}^{*} P_{1}\right]_{0}, \quad\left[P_{2}\right]_{[h], K^{d(p)}}=\left[h_{\xi}^{*} P_{2}\right]_{0} \quad \text { for a.e. } \xi \in \mathbb{B}_{\epsilon / 3}^{l} .
$$

By the cellular approximation theorem, there exists a cellular map $\phi$ : $L \rightarrow M$ (the $\mathrm{CW}$-structure of $M$ is given by $h: K \rightarrow M$ ) which we may assume Lipschitz such that $\phi$ is homotopic to $\varphi$. By definition, we have

$$
\left[P_{1}\right]_{[\phi], L}=\left[\phi_{\xi}^{*} P_{1}\right]_{0}, \quad\left[P_{2}\right]_{[\phi], L}=\left[\phi_{\xi}^{*} P_{2}\right]_{0} \quad \text { for a.e. } \xi \in \mathbb{B}_{\epsilon / 3}^{l} .
$$

For $\xi \in \mathbb{B}_{\epsilon}^{l}$, define $\iota_{\xi}(x)=\pi_{M}(x+\xi)$. Then we have $\phi_{\xi}=\iota_{\xi} \circ \phi$ and

$$
\phi_{\xi}^{*} P_{1}=\phi^{*} \iota_{\xi}^{*} P_{1}, \quad \phi_{\xi}^{*} P_{2}=\phi^{*} \iota_{\xi}^{*} P_{2} \quad \text { for a.e. } \xi \in \mathbb{B}_{\epsilon / 3}^{l} .
$$

By assumption and (3.11), we have

$$
\left[h^{*} \iota_{\xi}^{*} P_{1}\right]_{0}=\left[h_{\xi}^{*} P_{1}\right]_{0}=\left[h_{\xi}^{*} P_{2}\right]_{0}=\left[h^{*} \iota_{\xi}^{*} P_{2}\right]_{0} \quad \text { for a.e. } \xi \in \mathbb{B}_{\epsilon / 3}^{l} .
$$

Since $h: K \rightarrow M$ is a homeomorphism and $\iota_{\xi}^{*} P_{i} \in \mathcal{P}_{G}^{0}\left(M^{d(p)}\right)$ for a.e. $\xi \in \mathbb{B}_{\epsilon / 3}^{l}$ and $i=1,2$ (for $p=3$, they are defined as the $C^{0}$-isomorphism classes associated to $\left.\iota_{\xi} P_{i} \in \mathcal{P}_{G}^{1,3}\left(M^{3}\right)(i=1,2)\right)$, we have from (3.14) that

$$
\iota_{\xi}^{*} P_{1} \cong \iota_{\xi}^{*} P_{2} \quad \text { for a.e. } \xi \in \mathbb{B}_{\epsilon / 3}^{l} .
$$

From (3.12), (3.13) and (3.15), we obtain

$$
\left[P_{1}\right]_{[\varphi], L}=\left[P_{1}\right]_{[\phi], L}=\left[P_{2}\right]_{[\phi], L}=\left[P_{2}\right]_{[\varphi], L} .
$$

This completes the proof.

As remarked in Remark 3.1, we do not know whether the result presented in this subsection is optimal or not, i.e., we do not know whether the result analogous to Theorem 3.1 holds for $h: K \rightarrow M$ with $\operatorname{dim} K=[p]$. In the following subsections $\S 3.2$ and $\S 3.3$, we show that Theorem 3.1 is improved for bundles in the classes $\mathcal{P}_{\text {strong, },}^{k, p}(M)$ and $\mathcal{P}_{\text {weak }, G}^{k, p}(M)$. 
3.2. Topological invariants for $P \in \mathcal{P}_{\text {strong, },}^{k, p}(M)$

Here we consider bundles in the class $\mathcal{P}_{\text {strong, } G}^{k, p}(M)$. Since $\mathcal{P}_{\text {strong, } G}^{k, p}(M) \subset$ $\mathcal{P}_{\text {strong, } G}^{1, k p}(M)$, as in the previous subsection we consider only the case $k=1$, i.e., bundles in the class $\mathcal{P}_{\text {strong, } G}^{1, p}(M)$.

For $p \geq 1$, define $d_{\text {strong }}(p)=[p]$. Let $K$ be a polyhedron satisfying (A-1) and (A-2) in the appendix with $\operatorname{dim} K \leq d_{\text {strong }}(p)$ and $h: K \rightarrow M$ a Lipschitz map. As in the case of bundles in the class $\mathcal{P}_{G}^{1, p}(M)$, we want to associate the isomorphism class $[P]_{h, K} \in \hat{\mathcal{P}}_{G}^{0}(K)$ for $P \in \mathcal{P}_{\text {strong, } G}^{1, p}(M)$. The following is the main result in this section:

Theorem 3.2 Let $p \geq 1$. Let $P=\left\langle\left\{U_{\alpha}\right\},\left\{g_{\alpha \beta}\right\}\right\rangle \in \mathcal{P}_{\text {strong, } G}^{1, p}(M)$. Let $K$ be a polyhedron satisfying (A-1), (A-2) in the appendix and $h: K \rightarrow M a$ Lipschitz map. Assume that $\operatorname{dim} K \leq d_{\text {strong }}(p)$. For them there corresponds a class $[P]_{h, K} \in \hat{\mathcal{P}}_{G}^{0}(K)$. Moreover, this class depends only on the homotopy class of $h: K \rightarrow M$ and the $W^{1, p}$-isomorphism class of $P$ and coincides with the $C^{0}$-isomorphism class associated to $h_{\xi}^{*} P \in \mathcal{P}_{G}^{1, p}(K),\left[h_{\xi}^{*} P\right]_{0} \in \hat{\mathcal{P}}_{G}^{0}(K)$, for a.e. $\xi \in \mathbb{B}_{\epsilon / 3}^{l}$.

In this theorem, the $W^{1, p}$-isomorphism class of $P$ is the class of $P$ which we consider as a bundle in $\mathcal{P}_{G}^{1, p}(M)$. The key to the proof of the above theorem is the following lemma:

Lemma 3.9 Let $K, h$ be as above and $\left\{W_{p}\right\}_{p \in I}$ an open covering of $M$. Then there exists $\delta>0$ such that the following holds: Let $P, Q \in \mathcal{P}_{G}^{\infty}(M)$ be principal $G$-bundles of class $C^{\infty}$ such that they are trivializable over $W_{p}$ for all $p \in I$. Write $P=\left\langle\left\{W_{p}\right\},\left\{g_{p q}\right\}\right\rangle$ and $Q=\left\langle\left\{W_{p}\right\},\left\{h_{p q}\right\}\right\rangle$ and assume $\left\|g_{p q}-h_{p q}\right\|_{W^{1, p}\left(W_{p q}\right)}<\delta$ for all $p, q \in I$ with $W_{p q}:=W_{p} \cap W_{q} \neq \emptyset$. Then $h^{*} P \cong h^{*} Q$ as bundles over $K$.

Proof. By Lemma 3.1, there exists $\xi \in \mathbb{B}_{\epsilon}^{l}$ such that

$$
\left\|g_{p q} \circ h_{\xi}-h_{p q} \circ h_{\xi}\right\|_{W^{1, p}\left(h_{\xi}^{-1}\left(W_{p q}\right)\right)} \leq C \delta
$$

for some $C>0$ depending only on $M, G, K$ and $h$.

By the result proved in $[13, \S 3]$ and the appendix, $\S 7.1,(3.17)$ implies that

$$
h_{\xi}^{*} P \cong h_{\xi}^{*} Q
$$

over $K$ if $\delta>0$ is small.

Since $h_{\xi}$ is homotopic to $h$ by the homotopty $(t, x) \mapsto h_{t \xi}$, we see that

$$
h_{\xi}^{*} P \cong h^{*} P, \quad h_{\xi}^{*} Q \cong h^{*} Q
$$

over $K$. By (3.18) and (3.19), we obtain $h^{*} P \cong h^{*} Q$ over $K$. This completes the proof. 
Assume $\left\{P_{n}\right\} \subset \mathcal{P}_{G}^{\infty}(M)$ is an approximating sequence of $P \in \mathcal{P}_{\text {strong, } G}^{1, p}(M)$ as in Definition 2.1 (2). From Lemma 3.9 we see that the isomorphism class of $h^{*} P_{n} \in \mathcal{P}_{G}^{0}(M)$ is independent of $n$ for large $n$. The next lemma shows that this isomorphism class is also independent of the chosen approximation, namely, we have:

Lemma 3.10 Let $K, h$ be as in Lemma 3.9. Also let $p \geq 1$ and $P \in$ $\mathcal{P}_{\text {strong, } G}^{1, p}(M)$. Let $P_{n}=\left\langle\left\{V_{i}\right\},\left\{g_{i j}^{n}\right\}\right\rangle \in \mathcal{P}_{G}^{\infty}(M)$ and $Q_{n}=\left\langle\left\{W_{p}\right\},\left\{h_{p q}^{n}\right\}\right\rangle \in$ $\mathcal{P}_{G}^{\infty}(M)$ be approximating sequences of $P$ in the sense of Definition 2.1 (2), i.e., $\left\{V_{i}\right\}$ and $\left\{W_{p}\right\}$ are refinements of $M, V_{i} \subset U_{\varphi(i)}$ and $W_{p} \subset U_{\psi(p)}$, and $\left\|g_{\varphi(i) \varphi(j)}-g_{i j}^{n}\right\|_{W^{1, p}\left(V_{i j}\right)} \rightarrow 0$ and $\left\|g_{\psi(p) \psi(q)}-h_{p q}^{n}\right\|_{W^{1, p}\left(W_{p q}\right)} \rightarrow 0$ as $n \rightarrow \infty$. Then for large $n$, we have $h^{*} P_{n} \cong h^{*} Q_{n}$.

Proof. By Lemma 3.1 and the Fatou's lemma, there exist subsequences of $\left\{g_{i j}^{n}\right\}$ and $\left\{h_{p q}^{n}\right\}$ which we still denote by the same sequences and $\xi \in \mathbb{B}_{\epsilon / 3}^{l}$ of positive measure such that

$$
\left\|g_{\varphi(i) \varphi(j)} \circ h_{\xi}-g_{i j}^{n} \circ h_{\xi}\right\|_{W^{1, p}\left(h_{\xi}^{-1}\left(V_{i j}\right)\right)} \rightarrow 0
$$

and

$$
\left\|g_{\psi(p) \psi(q)} \circ h_{\xi}-h_{p q}^{n} \circ h_{\xi}\right\|_{W^{1, p}\left(h_{\xi}^{-1}\left(W_{p q}\right)\right)} \rightarrow 0
$$

as $n \rightarrow \infty$.

From $(3.20),(3.21)$ and the result in $[13, \S 3]$ and $\S 7.1$, we have

$$
\left[h_{\xi}^{*} P_{\left\{V_{i}\right\}}\right]_{0}=\left[h_{\xi}^{*} P_{n\left\{V_{i}\right\}}\right]_{0}
$$

and

$$
\left[h_{\xi} P_{\left\{W_{p}\right\}}\right]_{0}=\left[h_{\xi}^{*} Q_{n\left\{W_{p}\right\}}\right]_{0}
$$

for large $n$ and a.e. $\xi \in \mathbb{B}_{\epsilon / 3}^{l}$, where

$$
P_{\left\{V_{i}\right\}}:=\left\langle\left\{V_{i}\right\},\left\{g_{\varphi(i) \varphi(j)}\right\}\right\rangle \quad \text { and } \quad P_{\left\{W_{p}\right\}}:=\left\langle\left\{W_{p}\right\},\left\{g_{\psi(p) \psi(q)}\right\}\right\rangle
$$

are bundles in the class $\mathcal{P}_{\text {strong, } G}^{1, p}(M)$ and $\left[h_{\xi}^{*} P_{\left\{V_{i}\right\}}\right]_{0},\left[h_{\xi}^{*} P_{\left\{W_{p}\right\}}\right]_{0}$ denote the $C^{0}$ isomorphism classes associated to the bundles $h_{\xi}^{*} P_{\left\{V_{i}\right\}}, h_{\xi}^{*} P_{\left\{W_{p}\right\}} \in \mathcal{P}_{G}^{1, p}(K)$, respectively. $h_{\xi}^{*} P_{n\left\{V_{i}\right\}}$ and $h_{\xi}^{*} Q_{n\left\{W_{p}\right\}}$ are defined similarly.

Since the $C^{0}$-isomorphism class associated to a bundle in $\mathcal{P}_{G}^{1, p}(K)$ does not change if we pass to a refinement (see [13]), we have $\left[h_{\xi}^{*} P\right]_{0}=\left[h_{\xi}^{*} P_{\left\{V_{i}\right\}}\right]_{0}$ and $\left[h_{\xi}^{*} P\right]_{0}=\left[h_{\xi}^{*} P_{\left\{W_{p}\right\}}\right]_{0}$ for a.e. $\xi \in \mathbb{B}_{\epsilon / 3}^{l}$. Thus we obtain

$$
\left[h_{\xi}^{*} P_{\left\{V_{i}\right\}}\right]_{0}=\left[h_{\xi}^{*} P_{\left\{W_{p}\right\}}\right]_{0}
$$

for a.e. $\xi \in \mathbb{B}_{\epsilon / 3}^{l}$. 
From (3.22), (3.23) and (3.24), we have

$$
\left[h_{\xi}^{*} P_{n\left\{V_{i}\right\}}\right]_{0}=\left[h_{\xi}^{*} Q_{n\left\{W_{p}\right\}}\right]_{0} .
$$

Since $\left[h_{\xi}^{*} P_{n\left\{V_{i}\right\}}\right]_{0}=\left[h_{\xi}^{*} P_{n}\right]_{0}=\left[h^{*} P_{n}\right]_{0}$ and $\left[h_{\xi}^{*} Q_{n\left\{W_{p}\right\}}\right]_{0}=\left[h_{\xi}^{*} Q_{n}\right]_{0}=\left[h^{*} Q_{n}\right]_{0}$, we finally obtain $\left[h^{*} P_{n}\right]_{0}=\left[h^{*} Q_{n}\right]_{0}$ for large $n$.

By Lemma 3.10, the isomorphism class $h^{*} P_{n}$ for large $n$ is independent of the particular approximation of $P \in \mathcal{P}_{\text {strong, } G}^{1, p}(M)$ and the following definition is meaningful.

Definition 3.3 Let $P \in \mathcal{P}_{\text {strong, },}^{1, p}(M)$ and $\left\{P_{n}\right\}$ an approximating sequence of $P$ in the sense of Definition 2.1 (2). Also let $K$ be a polyhedron satisfying (A-1), (A-2) in the appendix, $\operatorname{dim} K \leq d_{\text {strong }}(p)$ and $h: K \rightarrow M$ a Lipschitz map. For large $n$, the isomorphism class $\left[h^{*} P_{n}\right]_{0} \in \mathcal{P}_{G}^{0}(K)$ is independent of $n$ and the approximating sequence $\left\{P_{n}\right\}$ and we denote this class by $[P]_{h, K}^{s}$.

To complete the proof of Theorem 3.2, we next show that the class $[P]_{h, K}^{s}$ depends only on the $W^{1, p}$-isomorphism class of $P$.

Lemma 3.11 Let $h: K \rightarrow M$ be as in Definition 3.3. Also let us assume that $P=\left\langle\left\{U_{\alpha}\right\}_{\alpha \in I},\left\{g_{\alpha \beta}\right\}_{\alpha \beta \in I}\right\rangle \in \mathcal{P}_{\text {strong, } G}^{1, p}(M)$ and $Q=\left\langle\left\{V_{i}\right\}_{i \in J},\left\{h_{i j}\right\}_{i, j \in J}\right\rangle \in$ $\mathcal{P}_{\text {strong, } G}^{1, p}(M)$ are $W^{1, p}$-isomorphic to each other as bundles in the class $\mathcal{P}_{G}^{1, p}(M)$ (see Definition 2.2). Then we have $[P]_{h, K}^{s}=[Q]_{h, K}^{s}$.

Proof. We first observe from the proof of Lemma 3.10 that $[P]_{h, K}^{s}$ and $[Q]_{h, K}^{s}$ are defined as the associated $C^{0}$-isomorphism classes of $h_{\xi}^{*} P \in \mathcal{P}_{G}^{1, p}(K)$ and $h_{\xi}^{*} Q \in \mathcal{P}_{G}^{1, p}(K)$ respectively, for a.e. $\xi \in \mathbb{B}_{\epsilon / 3}^{l}$.

By the assumption, for a.e. $\xi \in \mathbb{B}_{\epsilon / 3}^{l}$ we have (see Definition 2.2)

$$
g_{\varphi(s) \varphi(t)} \circ h_{\xi}=\left(\rho_{s} \circ h_{\xi}\right) \cdot\left(h_{\psi(s) \psi(t)} \circ h_{\xi}\right) \cdot\left(\rho_{t} \circ h_{\xi}\right)^{-1} \quad \text { in } h_{\xi}^{-1}\left(W_{s t}\right) .
$$

By the result of [13], we already know that the $C^{0}$-isomorphism class associated with a bundle in $\mathcal{P}_{G}^{1, p}(K)$ depends only on its $W^{1, p}$-isomorphism class (strictly speaking, we have only proved this for $K$ a manifold, but the proof equally applies for the present case once we have the result in $\S 7.1)$. Thus from (3.26), we have $\left[h_{\xi}^{*} P\right]_{0}=\left[h_{\xi}^{*} Q\right]_{0}$ for a.e. $\xi \in \mathbb{B}_{\epsilon / 3}^{l}$. Therefore by the remark given at the beginning of the proof, we obtain $[P]_{h, K}^{s}=[Q]_{h, K}^{s}$.

Notice that for $P \in \mathcal{P}_{\text {strong, } G}^{1, p}(M)$ and $\rho=\left\{\rho_{t}\right\}$ a $W^{1, p}$-bundle isomorphism (as in Definition 2.2), the gauge transformed bundle $\rho^{*} P:=$ $\left\langle\left\{W_{t}\right\},\left\{\rho_{s} \cdot g_{\varphi(s) \varphi(t)} \cdot \rho_{t}^{-1}\right\}\right\rangle$ is not a bundle in the class $\mathcal{P}_{\text {strong, } G}^{1, p}(M)$ in general. However, by Proposition 2.1 if $\rho_{t} \in W_{\text {strong }}^{1, p}\left(W_{s}, G\right)$, then $g^{*} P$ also 
belongs to $\mathcal{P}_{\text {strong, },}^{1, p}(M)$. The above lemma says that once we have $\rho^{*} P \in$ $\mathcal{P}_{\text {strong, } G}^{1, p}(M)$ for $P \in \mathcal{P}_{\text {strong, },}^{1, p}(M)$ and $\rho=\left\{\rho_{t}\right\}$ a $W^{1, p}$-bundle isomorphism, we have $\left[\rho^{*} P\right]_{h, K}^{s}=[P]_{h, K}^{s}$.

The final ingredient to complete the proof of Theorem 3.2 is

Lemma 3.12 Let $K$ be a polyhedron satisfying (A-1), (A-2) in the appendix, $\operatorname{dim} K \leq d_{\text {strong }}(p)$ and $h_{1}, h_{2}: K \rightarrow M$ two Lipschitz maps such that they are homotopic to each other. Then we have $[P]_{h_{1}, K}^{s}=[P]_{h_{2}, K}^{s}$.

Proof. Let $P_{n} \in \mathcal{P}_{G}^{\infty}(M)$ be an approximating sequence of $P$ in the sense of Definition 2.1 (2). By definition, we have $[P]_{h_{1}, K}^{s}=\left[h_{1}^{*} P_{n}\right]_{0}$ and $[P]_{h_{2}, K}^{s}=$ $\left[h_{2}^{*} P_{n}\right]_{0}$ for large $n$. Since $h_{1}$ is homotopic to $h_{2}$, we have $h_{1}^{*} P_{n} \cong h_{2}^{*} P_{n}$ for all $n$. Thus we have $[P]_{h_{1}, K}^{s}=[P]_{h_{2}, K}^{s}$. This completes the proof.

Combining Lemma 3.11 and Lemma 3.12, we complete the proof of Theorem 3.2 .

Definition 3.4 Let $P \in \mathcal{P}_{\text {strong, } G}^{1, p}(M)$ and $h: K \rightarrow M$ a Lipschitz map with $K$ satisfying (A-1), (A-2) in the appendix and $\operatorname{dim} K \leq d_{\text {strong }}(p)$. Since the class $[P]_{h, K}^{s}$ depends only on the homotopy class $[h] \in[K, M]$, we denote this as $[P]_{[h], K}^{s}$.

As we remarked before Lemma 3.12, $W^{1, p}$-isomorphism $\rho=\left\{\rho_{s}\right\}$ with $\rho_{s} \in W_{\text {strong }}^{1, p}\left(W_{s}, G\right)$ acts on $\mathcal{P}_{\text {strong, } G}^{1, p}(M)$. From this we have the following corollary (which simply restate Theorem 3.2, but the assertion is somewhat weaker):

Corollary 3.1 Let $h: K \rightarrow M$ be a Lipschitz map with $K$ satisfying (A-1) and (A-2) in the appendix and $\operatorname{dim} K \leq d_{\text {strong }}(p)$. Then there exists a homomorphism

$$
h^{*}: H^{1}\left(M ; \mathcal{W}_{\text {strong }, G}^{1, p}\right) \rightarrow H^{1}\left(K ; \bigodot_{G}^{0}\right)
$$

defined by $h^{*}\left([P]_{1, p}^{s}\right)=[P]_{h, K}^{s}$, where $[P]_{1, p}^{s} \in H^{1}\left(M ; \mathcal{W}_{\text {strong, },}^{1, p}\right)=\hat{\mathcal{P}}_{\text {strong,G }}^{1, p}(M)$ denotes the isomorphism class of $P$. Moreover, $h^{*}$ depends only on the homotopy class of $h$.

\subsection{Topological invariants for $P \in \mathcal{P}_{\text {weak }, G}^{k, p}(M)$}

Since $\mathcal{P}_{\text {weak,G }}^{k, p}(M) \subset \mathcal{P}_{\text {weak, } G}^{1, k p}(M)$ as in the previous sections, we only consider the case $k=1$. The idea for defining invariants for $P \in \mathcal{P}_{\text {weak, } G}^{1, p}(M)$ is very similar to the previous subsection.

For $p \geq 1$, define $d_{\text {weak }}(p)=$ the largest integer strictly less than $p$. Notice that, by the Sobolev embedding, we have $\mathcal{P}_{G}^{1, p}(K) \subset \mathcal{P}_{G}^{0}(M)$ when $\operatorname{dim} K \leq d_{\text {weak }}(p)$. Our main result is the following: 
Theorem 3.3 Assume $p \geq 1$. Let $K$ be a polyhedron satisfying (A-1), (A-2) in the appendix and $h: K \rightarrow M$ a Lipschitz map. Assume that $\operatorname{dim} K \leq$ $d_{\text {weak }}(p)$. Then for $P=\left\langle\left\{U_{\alpha}\right\},\left\{g_{\alpha \beta}\right\}\right\rangle \in \mathcal{P}_{\text {weak, },}^{1, p}(M)$, there corresponds a class $[P]_{h, K} \in \hat{\mathcal{P}}_{G}^{0}(K)$. Moreover, this class depends only on the homotopy class of $h: K \rightarrow M$ and the $W^{1, p}$-isomorphism class of $P$ and coincides with the $C^{0}$-class of $h_{\xi}^{*} P \in \mathcal{P}_{G}^{1, p}(K) \subset \mathcal{P}_{G}^{0}(K),\left[h_{\xi}^{*} P\right]_{0} \in \hat{\mathcal{P}}_{G}^{0}(K)$, for a.e. $\xi \in \mathbb{B}_{\epsilon / 3}^{l}$.

In this theorem, the $W^{1, p}$-isomorphism class is taken as that of Theorem 3.2. The key step for the proof of the above theorem is the following:

Lemma 3.13 Let $h: K \rightarrow M$ be as above, $\left\{U_{\alpha}\right\}$ an open covering of $M$ and $D>0$ a constant. There exists $\delta>0$ depending only on $h: K \rightarrow M,\left\{U_{\alpha}\right\}$ and $D$ such that the following holds: Suppose $P \rightarrow M$ and $Q \rightarrow M$ are two principal $G$-bundles of class $C^{\infty}$ which are represented as $P=\left\langle\left\{U_{\alpha}\right\},\left\{g_{\alpha \beta}^{P}\right\}\right\rangle$ and $Q=\left\langle\left\{U_{\alpha}\right\},\left\{g_{\alpha \beta}^{Q}\right\}\right\rangle$ such that

$$
\left\|g_{\alpha \beta}^{P}-g_{\alpha \beta}^{Q}\right\|_{L^{p}\left(U_{\alpha \beta}\right)}<\delta, \quad\left\|d g_{\alpha \beta}^{P}\right\|_{L^{p}\left(U_{\alpha \beta}\right)},\left\|d g_{\alpha \beta}^{Q}\right\|_{L^{p}\left(U_{\alpha \beta}\right)} \leq D
$$

for any $\alpha, \beta$ with $U_{\alpha \beta} \neq \emptyset$. Then there holds $h^{*} P \cong h^{*} Q$.

Proof. By Lemma 3.1, there exist $\xi \in \mathbb{B}_{\epsilon}^{l}$ of positive measure and $C>0$ depending only on $h: K \rightarrow M$ and $G$ such that

$$
\left\|g_{\alpha \beta}^{P} \circ h_{\xi}-g^{Q} \circ h_{\xi}\right\|_{L^{p}\left(h_{\xi}^{-1}\left(U_{\alpha \beta}\right)\right)} \leq C \delta
$$

and

$$
\left\|d\left(g_{\alpha \beta}^{P} \circ h_{\xi}\right)\right\|_{L^{p}\left(h_{\xi}^{-1}\left(U_{\alpha \beta}\right)\right)},\left\|d\left(g_{\alpha \beta}^{Q} \circ h_{\xi}\right)\right\|_{L^{p}\left(h_{\xi}^{-1}\left(U_{\alpha \beta}\right)\right)} \leq C D .
$$

Notice that, by the Sobolev embedding, $h_{\xi}^{*} P=\left\langle\left\{h_{\xi}^{-1}\left(U_{\alpha \beta}\right)\right\},\left\{g_{\alpha \beta}^{P} \circ h_{\xi}\right\}\right\rangle$ and $h_{\xi}^{*} Q=\left\langle\left\{h_{\xi}^{-1}\left(U_{\alpha \beta}\right)\right\},\left\{g_{\alpha \beta}^{Q} \circ h_{\xi}\right\}\right\rangle$ are bundles of class $C^{0}$ for a.e. $\xi \in \mathbb{B}_{\epsilon}^{l}$. Also by the Sobolev embedding, (3.27) and (3.28), $\left\|g_{\alpha \beta}^{P} \circ h_{\xi}-g_{\alpha \beta}^{Q} \circ h_{\xi}\right\|_{C^{0}\left(h_{\xi}^{-1}\left(U_{\alpha \beta}\right)\right)}$ is small if $\delta>0$ is small. Thus if we choose $\delta>0$ small, we have $h_{\xi}^{*} P \cong h_{\xi} Q$ for $P \rightarrow M$ and $Q \rightarrow M$ satisfying the assumption of the lemma. Since $h_{\xi}$ is homotopic to $h$, we also have $h_{\xi} P \cong h^{*} P$ and $h_{\xi}^{*} Q \cong h^{*} Q$. Therefore we have $h^{*} P \cong h^{*} Q$. This completes the proof.

Let $P \in \mathcal{P}_{\text {weak, } G}^{1, p}(M)$ and $\left\{P_{n}\right\} \subset \mathcal{P}_{G}^{\infty}(M)$ be an approximating sequence of $P$ in the sense of Definition 2.1 (3). By Lemma 3.13, the isomorphism class of $h^{*} P_{n}$ is independent of $n$ if $n$ is large. As in the case of bundles in $\mathcal{P}_{\text {strong, } G}^{1, p}(M)$, we want to define the class $\left[h^{*} P_{n}\right]_{0}$ for large $n$ as the class of the "bundle" $h^{*} P$ for $P \in \mathcal{P}_{\text {weak }, G}^{1, p}(M)$. For this, we need to show that the class $\left[h^{*} P_{n}\right]_{0} \in \hat{\mathcal{P}}_{G}^{0}(M)$ does not depend on the specific choice of the approximation. 
Lemma 3.14 Let $P=\left\langle\left\{U_{\alpha}\right\},\left\{g_{\alpha \beta}\right\}\right\rangle \in \mathcal{P}_{\text {weak, },}^{1, p}(M)$ and $h: K \rightarrow M$ a Lipschitz map, where $K$ is a polyhedron satisfying (A-1), (A-2) in the appendix with $\operatorname{dim} K \leq d_{\text {weak }}(p)$. Suppose

$$
P_{n}=\left\langle\left\{V_{i}\right\},\left\{g_{i j}^{n}\right\}\right\rangle \in \mathcal{P}_{G}^{\infty}(M) \quad \text { and } \quad Q_{n}=\left\langle\left\{W_{p}\right\},\left\{h_{p q}^{n}\right\}\right\rangle \in \mathcal{P}_{G}^{\infty}(M)
$$

are two approximating sequences of $P$ in the sense of Definition 2.1 (3). Then for large $n$, we have

$$
h^{*} P_{n} \cong h^{*} Q_{n} .
$$

Proof. By assumption, there exists $D>0$ such that

$$
\left\|g_{\varphi(i) \varphi(j)}-g_{i j}^{n}\right\|_{L^{p}\left(V_{i j}\right)} \rightarrow 0, \quad\left\|g_{\psi(p) \psi(q)}-h_{p q}^{n}\right\|_{L^{p}\left(W_{p q}\right)} \rightarrow 0
$$

as $n \rightarrow \infty$ and

$$
\left\|d g_{i j}^{n}\right\|_{L^{p}\left(V_{i j}\right)} \leq D, \quad\left\|d h_{p q}^{n}\right\|_{L^{p}\left(W_{p q}\right)} \leq D
$$

for all $i, j$ and $p, q$ with $V_{i j} \neq \emptyset$ and $W_{p q} \neq \emptyset$ respectively, where $\varphi$ and $\psi$ are refinement maps, i.e., $V_{i} \subset U_{\varphi(i)}$ and $W_{p} \subset U_{\psi(p)}$.

By Lemma 3.1, (3.29) and (3.30) imply that there exists $\xi \in \mathbb{B}_{\epsilon}^{l}$ of positive measure such that

$$
\begin{array}{r}
\left\|g_{\varphi(i) \varphi(j)} \circ h_{\xi}-g_{i j}^{n} \circ h_{\xi}\right\|_{L^{p}\left(h_{\xi}^{-1}\left(V_{i j}\right)\right)} \rightarrow 0, \\
\left\|g_{\psi(p) \psi(q)} \circ h_{\xi}-h_{p q}^{n} \circ h_{\xi}\right\|_{L^{p}\left(h_{\xi}^{-1}\left(W_{p q}\right)\right)} \rightarrow 0
\end{array}
$$

as $n \rightarrow \infty$ and

$$
\left\|d\left(g_{i j}^{n} \circ h_{\xi}\right)\right\|_{L^{p}\left(h_{\xi}^{-1}\left(V_{i j}\right)\right)} \leq C D, \quad\left\|d\left(h_{p q}^{n} \circ h_{\xi}\right)\right\|_{L^{p}\left(h_{\xi}^{-1}\left(W_{p q}\right)\right)} \leq C D
$$

for all $n$, where $C>0$ is a constant independent of $n$ and $\xi$.

Since $d_{\text {weak }}(p)<p$, by the Sobolev embedding $W^{1, p}(K) \subset C^{0}(K)$ and (3.31), (3.32) and (3.33) we have, by the same reasoning as in the proof of Lemma 3.13,

$$
h_{\xi}^{*} P_{\left\{V_{i}\right\}} \cong h_{\xi}^{*} P_{n} \cong h^{*} P_{n}
$$

and

$$
h_{\xi}^{*} P_{\left\{W_{p}\right\}} \cong h_{\xi}^{*} Q_{n} \cong h^{*} Q_{n}
$$

for large $n$. Here we notice that $h_{\xi}^{*} P_{\left\{U_{\alpha}\right\}}=\left\langle\left\{h_{\xi}^{-1}\left(U_{\alpha}\right)\right\},\left\{g_{\alpha \beta} \circ h_{\xi}\right\}\right\rangle$ defines a $C^{0}$-bundle for a.e. $\xi \in \mathbb{B}_{\epsilon}^{l}$ and since $\left\{h_{\xi}^{-1}\left(V_{i}\right)\right\}$ and $\left\{h_{\xi}^{-1}\left(W_{p}\right)\right\}$ are refinements of $\left\{h_{\xi}^{-1}\left(U_{\alpha}\right)\right\}$, we have

$$
h_{\xi}{ }^{*} P_{\left\{V_{i}\right\}} \cong h_{\xi}{ }^{*} P_{\left\{U_{\alpha}\right\}} \cong h_{\xi}^{*} P_{\left\{W_{p}\right\}} .
$$

From (3.34), (3.35) and (3.36), we obtain $h^{*} P_{n} \cong h^{*} Q_{n}$ for large $n$. 
In view of the above lemma, we give the following:

Definition 3.5 Let $P \in \mathcal{P}_{\text {weak, },}^{1, p}(M)$ and $h: K \rightarrow M$ a Lipschitz map with $K$ satisfying (A-1), (A-2) in the appendix and $\operatorname{dim} K \leq d_{\text {weak }}(p)$. Let $\left\{P_{n}\right\} \subset \mathcal{P}_{G}^{\infty}(M)$ be an approximating sequence of $P$ in the sense of Definition $2.1(3)$. For large $n$, the class $\left[h^{*} P_{n}\right]_{0} \in \hat{\mathcal{P}}_{G}^{0}(K)$ is independent of $n$ and the approximating sequence $\left\{P_{n}\right\}$ and we denote this class by $[P]_{h, K}^{w}$.

To complete the proof of Theorem 3.3, we next show that the class $[P]_{h, K}^{w}$ depends only on the $W^{1, p}$-isomorphism class of $P$. Namely, we show:

Lemma 3.15 Let $h: K \rightarrow M$ be a Lipschitz map with $K$ satisfying (A-1), (A-2) in the appendix and $\operatorname{dim} K \leq d_{\text {weak }}(p)$. Suppose $P=\left\langle\left\{U_{\alpha}\right\},\left\{g_{\alpha \beta}\right\}\right\rangle \in$ $\mathcal{P}_{\text {weak }, G}^{1, p}(M)$ and $Q=\left\langle\left\{V_{i}\right\},\left\{h_{i j}\right\}\right\rangle \in \mathcal{P}_{\text {weak, } G}^{1, p}(M)$ are $W^{1, p}$-isomorphic to each other as bundles in $\mathcal{P}_{G}^{1, p}(M)$ (see Definition 2.2). Then we have $[P]_{h, K}^{w}=[Q]_{h, K}^{w}$.

Proof. The proof of Lemma 3.14 shows that the classes $[P]_{h, K}^{w}$ and $[Q]_{h, K}^{w}$ are defined as the $C^{0}$-isomorphism classes of $h_{\xi}^{*} P \in \mathcal{P}_{G}^{0}(M)$ and $h_{\xi}^{*} Q \in \mathcal{P}_{G}^{0}(M)$ respectively, for a.e. $\xi \in \mathbb{B}_{\epsilon}^{l}$.

Let $\rho=\left\{\rho_{s}\right\}$ be as in Definition 2.2. Then for a.e. $\xi \in \mathbb{B}_{\epsilon}^{l}$, we have

$$
g_{\varphi(s) \varphi(t)} \circ h_{\xi}=\left(\rho_{s} \circ h_{\xi}\right) \cdot\left(h_{\psi(s) \psi(t)} \circ h_{\xi}\right) \cdot\left(\rho_{t} \circ h_{\xi}\right)^{-1}
$$

in $h_{\xi}^{-1}\left(W_{s t}\right)$.

From (3.37), we obtain $h_{\xi}^{*} P \cong h_{\xi}^{*} Q$ as $C^{0}$-bundles for a.e. $\xi \in \mathbb{B}_{\epsilon}^{l}$. By the remark at the beginning of the proof, this completes the proof.

The final ingredient to complete the proof of Theorem 3.3 is:

Lemma 3.16 Let $P \in \mathcal{P}_{\text {weak, },}^{1, p}(M)$ and $K$ a polyhedron satisfying $(\mathrm{A}-1),(\mathrm{A}-2)$ in the appendix and $\operatorname{dim} K \leq d_{\text {weak }}(P)$. Suppose that two Lipschitz maps $h_{1}, h_{2}: K \rightarrow M$ are homotopic to each other. Then we have $[P]_{h_{1}, K}^{w}=[P]_{h_{2}, K}^{w}$.

Proof. Let $\left\{P_{n}\right\} \subset \mathcal{P}_{\text {weak }, G}^{1, p}(M)$ be an approximating sequence of $P$ in the sense of Definition $2.1(3)$. By definition, we have $[P]_{h_{1}, K}^{w}=\left[h_{1}{ }^{*} P_{n}\right]_{0}$ and $[P]_{h_{2}, K}^{w}=\left[h_{2}{ }^{*} P_{n}\right]_{0}$ for large $n$. Since $h_{1}$ is homotopic to $h_{2}$, we have $h_{1}{ }^{*} P_{n} \cong h_{2}{ }^{*} P_{n}$ for all $n$. Thus we obtain $[P]_{h_{1}, K}^{w}=[P]_{h_{2}, K}^{w}$.

Combining Lemma 3.15 and Lemma 3.16, we complete the proof of Theorem 3.3.

Definition 3.6 Let $P \in \mathcal{P}_{\text {weak }, G}^{1, p}(M)$ and $h: K \rightarrow M$ a Lipschitz map with $K$ satisfying (A-1), (A-2) in the appendix and $\operatorname{dim} K \leq d_{\text {weak }}(p)$. Since the class $[P]_{h, K}^{w}$ depends only on the homotopy class $[h] \in[K, M]$, we denote this also as $[P]_{[h], K}^{w}$. 
Since the $W^{1, p}$-isomorphism $\rho=\left\{\rho_{s}\right\}$ with $\rho_{s} \in W_{\text {weak }}^{1, p}\left(W_{s}, G\right)$ acts on $\mathcal{P}_{\text {weak }, G}^{1, p}(M)$ by Proposition 2.1, we have the following corollary:

Corollary 3.2 Let $h: K \rightarrow M$ be a Lipschitz map with $K$ satisfying (A-1), (A-2) in the appendix and $\operatorname{dim} K \leq d_{\text {weak }}(p)$. Then there exists a homomorphism

$$
h^{*}: H^{1}\left(M ; \mathcal{W}_{\text {weak,G }}^{1, p}\right) \rightarrow H^{1}\left(K ; \mathrm{C}_{G}^{0}\right)
$$

defined by $h^{*}\left([P]_{1, p}\right)=[P]_{h, K}^{w}$, where $[P]_{1, p}^{w} \in H^{1}\left(M ; \mathcal{W}_{\text {weak,G }}^{1, p}\right)=\hat{\mathcal{P}}_{\text {weak }, G}^{1, p}(M)$ denotes the isomorphism class of $P . h^{*}$ depends only on the homotopy class of $h$.

\subsection{The case $G=\mathbb{T}^{k}$}

In this section, we consider the case $G=\mathbb{T}^{k}(k \geq 1)$ of torus bundles. In this case, we show that the results proved in the previous subsections are improved. Namely, we have

Theorem 3.4 Assume $G=\mathbb{T}^{k}$ for some $k \geq 1$ and $P=\left\langle\left\{U_{\alpha}\right\},\left\{g_{\alpha \beta}\right\}\right\rangle \in$ $\mathcal{P}_{G}^{1, p}(M)$. Let $K$ be a polyhedron satisfying (A-1), (A-2) in the appendix with arbitrary dimension and $h: K \rightarrow M$ a Lipschitz map. For $p \geq 3$, we have a class $[P]_{h, K} \in \hat{\mathcal{P}}_{G}^{0}(M)$ which depends only on the $W^{1, p}$-isomorphism class of $P$ and the homotopy class of $h$.

Proof. By Theorem 3.1, $P \in \mathcal{P}_{G}^{1, p}(M)$ defines uniquely a class $[P]_{h, K^{d(p)}} \in$ $\hat{\mathcal{P}}_{G}^{0}\left(K^{d(p)}\right)$ which depends only on the $W^{1, p}$-isomorphism class of $P$ and the homotopy class of $h$. By the inclusion $K^{d(p)} \subset K$, we have $\left[K, B \mathbb{T}^{k}\right] \rightarrow$ $\left[K^{d(p)}, B T^{k}\right]$. Since $B \mathbb{T}^{k} \simeq B \mathbb{T} \times \cdots \times B \mathbb{T} \simeq \mathbb{C} P^{\infty} \times \cdots \times \mathbb{C} P^{\infty}(k$-times $)$, $\mathbb{C} P^{\infty}=K(\mathbb{Z}, 2)$ and $[K, K(\mathbb{Z}, 2)] \cong H^{2}(K ; \mathbb{Z})$, etc., we obtain

$$
H^{2}(K ; \mathbb{Z}) \times \cdots \times H^{2}(K ; \mathbb{Z}) \rightarrow H^{2}\left(K^{d(p)} ; \mathbb{Z}\right) \times \cdots \times H^{2}\left(K^{d(p)} ; \mathbb{Z}\right) .
$$

On the other hand, by the cohomology exact sequence of the pair $\left(K, K^{d(p)}\right)$, we have

$$
\cdots \rightarrow H^{2}\left(K, K^{d(p)} ; \mathbb{Z}\right) \rightarrow H^{2}(K ; \mathbb{Z}) \rightarrow H^{2}\left(K^{d(p)} ; \mathbb{Z}\right) \rightarrow H^{3}\left(K, K^{d(p)} ; \mathbb{Z}\right) \rightarrow \cdots
$$

Since $d(p) \geq 3$ when $p \geq 3$, we have by the above sequence $H^{2}(K ; \mathbb{Z}) \simeq$ $H^{2}\left(K^{d(p)} ; \mathbb{Z}\right)$. This implies that the homomorphism given by $(3.38)$ is an isomorphism. In other words, we have an isomorphism $\check{H}^{1}\left(K^{d(p)} ; \bigodot_{G}^{0}\right) \cong$ $\check{H}^{1}\left(K ; \mathrm{C}_{G}^{0}\right)$. Composing this with the homomorphism $h^{*}: \check{H}^{1}\left(M ; \mathcal{W}_{G}^{1, p}\right) \rightarrow$ $\check{H}^{1}\left(K^{d(p)} ; \bigodot_{G}^{0}\right)$, we obtain

$$
\check{H}^{1}\left(M ; \mathcal{W}_{G}^{1, p}\right) \stackrel{h^{*}}{\rightarrow} \check{H}^{1}\left(K^{d(p)} ; \bigodot_{G}^{0}\right) \stackrel{\sim}{\rightarrow} \check{H}^{1}\left(K ; \bigodot_{G}^{0}\right) .
$$

Defining the image of $[P] \in \hat{\mathcal{P}}_{G}^{1, p}(M) \simeq \check{H}^{1}\left(M ; \mathcal{W}_{G}^{1, p}\right)$ under $(3.39)$ by $[P]_{h, K} \in$ $\mathcal{P}_{G}^{0}(K)$, we obtain the desired class. This completes the proof. 


\section{Chern-Weil theory for Sobolev connections on Sobo- lev bundles}

This section extends the classical Chern-Weil theory to Sobolev connections on Sobolev bundles. Since $\mathcal{P}_{G}^{k, p}(M) \subset \mathcal{P}_{G}^{1, k p}(M)$ for $k \geq 1$, we again only consider principal $G$-bundles of class $W^{1, p}$ and $\mathfrak{A}^{1, p / 2}$-connections.

Let $P=\left\langle\left\{U_{\alpha}\right\}_{\alpha \in I},\left\{g_{\alpha \beta}\right\}_{\alpha \beta \in I}\right\rangle \in \mathcal{P}_{G}^{1, p}(M)$ and $A=\left\{A_{\alpha}\right\} \in \mathfrak{A}^{1, p / 2}(P)$. Let $S^{k}\left(\mathfrak{g}^{*}\right) \subset\left(\mathfrak{g}^{*}\right)^{\otimes k}$ denote the symmetric power, i.e.,

$$
\phi \in S^{k}\left(\mathfrak{g}^{*}\right) \quad \text { if } \quad \phi: \underbrace{\mathfrak{g} \times \cdots \times \mathfrak{g}}_{k} \rightarrow \mathbb{R} \quad \text { is symmetric multilinear. }
$$

The adjoint action of $G$ on $\mathfrak{g}$ induces a $G$-action on $S^{k}\left(\mathfrak{g}^{*}\right)$. We denote by $I^{k}(G)$ the $G$-invariant elements of $S^{k}\left(\mathfrak{g}^{*}\right)$. That is, $\phi \in I^{k}(G)$ if $\phi\left(\operatorname{Ad}(g) X_{1}, \ldots, \operatorname{Ad}(g) X_{k}\right)=\phi\left(X_{1}, \ldots, X_{k}\right)$ for $g \in G$ and $X_{1}, \ldots, X_{k} \in \mathfrak{g}$. For $\phi \in S^{k}\left(\mathfrak{g}^{*}\right), x \in M$ and $\omega_{i} \otimes X_{i} \in \bigwedge^{\text {even }} T_{x}^{*} M \otimes \mathfrak{g}(i=1, \ldots, k)$, we define

$$
\phi\left(\omega_{1} \otimes X_{1}, \ldots, \omega_{k} \otimes X_{k}\right)=\phi\left(X_{1}, \ldots, X_{k}\right) \omega_{1} \wedge \cdots \wedge \omega_{k} \in \bigwedge^{\bullet} T_{x}^{*} M .
$$

Let $\phi \in I^{k}(G)$. We assume in the following $p / 2 \geq k$. Since $F_{A_{\alpha}} \in$ $L^{p / 2}\left(U_{\alpha}, \bigwedge^{2} T^{*} U_{\alpha} \otimes \mathfrak{g}\right)$, we have

$$
P_{\phi}\left(F_{A_{\alpha}}\right):=\phi\left(F_{A_{\alpha}}, \ldots, F_{A_{\alpha}}\right) \in L^{p / 2 k}\left(U_{\alpha}, \bigwedge^{2 k} T^{*} U_{\alpha}\right) \subset L^{1}\left(U_{\alpha}, \bigwedge^{2 k} T^{*} U_{\alpha}\right) \text {. }
$$

By the $G$-invariance of $\phi$, different $P_{\phi}\left(F_{\alpha}\right)$ are glued together to give a global $2 k$-form in $L^{p / 2 k}\left(M, \bigwedge^{2 k} T^{*} M\right)$ which we denote by $P_{\phi}\left(F_{A}\right)$.

For smooth $P$ and $A$, the classical Chern-Weil theory asserts (see [15]) that i) the form $P_{\phi}\left(F_{A}\right)$ is closed, ii) the cohomology class of $P_{\phi}\left(F_{A}\right)$ is independent of $A$. We extend these for $P \in \mathcal{P}_{G}^{1, p}(M)$ and $A \in \mathfrak{A}^{1, p / 2}(P)$.

Before beginning, we first observe that we may assume $p<m$ since the case $p \geq m$ has been treated in our previous paper [13]. Then, since $2 k+1 \leq p+1<m+1$, we have $2 k+1 \leq m$ and the form $P_{\phi}\left(F_{A}\right)$ is a form of degree $2 k \leq m-1$ on $M$.

Under the above assumption, our first lemma asserts that $P_{\phi}\left(F_{A}\right)$ is closed in the sense of distributions:

Lemma 4.1 Let $P=\left\langle\left\{U_{\alpha}\right\},\left\{g_{\alpha \beta}\right\}\right\rangle \in \mathcal{P}_{G}^{1, p}(M)(p \geq 2)$ and $A=\left\{A_{\alpha}\right\} \in$ $\mathfrak{A}^{1, p / 2}(P)$. Let $k \leq p / 2$ be a positive integer and $\phi \in I^{k}(G)$ an invariant polynomial. Then we have $d P_{\phi}\left(F_{A}\right)=0$ in $\mathcal{D}^{\prime}\left(M, \bigwedge^{2 k+1} T^{*} M\right)$, i.e., we have

$$
\int_{M} P_{\phi}\left(F_{A}\right) \wedge d \alpha=0
$$

for any $\beta \in C^{\infty}\left(M, \bigwedge^{m-2 k-1} T^{*} M\right)$. 
Proof. Since the assertion $d P_{\phi}\left(F_{A}\right)=0$ in $\mathcal{D}^{\prime}\left(M, \bigwedge^{2 k+1} T^{*} M\right)$ is local, i.e., if it is satisfied on any $U_{\alpha}$, by the partition of unity, it holds on $M$. So it suffices to prove (4.1) for $\beta \in C^{\infty}\left(M, \bigwedge^{m-2 k-1} T^{*} M\right)$ with $\operatorname{supp}(\beta) \subset U_{\alpha}$.

On $U_{\alpha}$, we have $P_{\phi}\left(F_{A}\right)=P_{\phi}\left(F_{A_{\alpha}}\right)$. Since $A \in \mathfrak{A}^{1, p / 2}(P)$, i.e., $A_{\alpha} \in$ $L^{p}\left(U_{\alpha}\right)$ and $d A_{\alpha} \in L^{p / 2}\left(U_{\alpha}\right)$, there exists $\left\{A_{\alpha, n}\right\} \subset C^{\infty}\left(U_{\alpha}, T^{*} U_{\alpha} \otimes \mathfrak{g}\right)$ such that $A_{\alpha, n} \rightarrow A_{\alpha}$ in $L^{p}\left(U_{\alpha}\right)$ and $d A_{\alpha, n} \rightarrow d A_{\alpha}$ in $L^{p / 2}\left(U_{\alpha}\right)$ as $n \rightarrow \infty$. From these, we have

$$
P_{\phi}\left(F_{A_{\alpha, n}}\right) \rightarrow P_{\phi}\left(F_{A_{\alpha}}\right)
$$

in $L^{p / 2 k}\left(U_{\alpha}\right)$, in particular, in $L^{1}\left(U_{\alpha}\right)$.

By the Bianchi identity, we have $d_{A_{\alpha, n}} F_{A_{\alpha, n}}=0$ and $d P_{\phi}\left(F_{A_{\alpha, n}}\right)=0$. Thus we have by the Stokes theorem

$$
\int_{M} P_{\phi}\left(F_{A_{\alpha, n}}\right) \wedge d \beta=0
$$

for all $\beta \in C^{\infty}\left(M, \bigwedge^{m-2 k-1} T^{*} M\right)$ with $\operatorname{supp}(\beta) \subset U_{\alpha}$. Letting $n \rightarrow \infty$ in (4.3), we have by $(4.2)$

$$
\int_{M} P_{\phi}\left(F_{A_{\alpha}}\right) \wedge d \alpha=0
$$

This completes the proof.

Let $P=\left\langle\left\{U_{\alpha}\right\},\left\{g_{\alpha \beta}\right\}\right\rangle \in \mathcal{P}_{G}^{1, p}(M), A=\left\{A_{\alpha}\right\} \in \mathfrak{A}^{1, p / 2}(P)$ and $\phi \in I^{k}(G)$ with $p / 2 \geq k$ be as before. Also let $S$ be an arbitrary closed manifold and $f: S \rightarrow M$ a Lipschitz map. We next want to consider the pulled back form $f^{*} P_{\phi}\left(F_{A}\right)$. We first remark that even though $P_{\phi}\left(F_{A}\right) \in L^{1}\left(M, \bigwedge^{2 k} T^{*} M\right)$, $f^{*} P_{\phi}\left(F_{A}\right)$ does not belong to $L^{1}\left(S, \bigwedge^{2 k} T^{*} S\right)$ in general. This is because the composition $g \circ h$ of a $L^{1}$-function $g$ with a Lipschitz map $h$ does not preserve the $L^{1}$-property, i.e., $g \circ h \notin L^{1}$ in general. However, we shall show that $f^{*} P_{\phi}\left(F_{A}\right)$ belongs to $L^{1}$ and satisfies $d\left(f^{*} P_{\phi}\left(F_{A}\right)\right)=0$ in $\mathcal{D}^{\prime}\left(S, \bigwedge^{2 k+1} T^{*} S\right)$ for generic $f$. That is, we have

Lemma 4.2 Let $P=\left\langle\left\{U_{\alpha}\right\},\left\{g_{\alpha \beta}\right\}\right\rangle \in \mathcal{P}_{G}^{1, p}(M), A=\left\{A_{\alpha}\right\} \in \mathfrak{A}^{1, p / 2}(P)$ and $\phi \in I^{k}(G)$. Also let $S$ be an arbitrary closed manifold of dimension $i$ and $f: S \rightarrow M$ a Lipschitz map. Suppose $p / 2 \leq k$, then for a.e. $\xi \in \mathbb{B}_{\epsilon}^{l}$ we have $f_{\xi}^{*} P_{\phi}\left(F_{A}\right) \in L^{1}\left(S, \bigwedge^{2 k} T^{*} S\right)$ and

$$
d\left(f_{\xi}^{*} P_{\phi}\left(F_{A}\right)\right)=0 \quad \text { in } \mathcal{D}^{\prime}\left(S, \bigwedge^{2 k+1} T^{*} S\right) .
$$


Proof. Since $f: S \rightarrow M$ is Lipschitz, there exists a sequence of smooth maps $\left\{f_{n}\right\} \subset C^{\infty}(S, M)$ such that $f_{n} \rightarrow f$ in $\operatorname{Lip}\left(S, \mathbb{R}^{l}\right)$, i.e.,

$\sup _{x \in S}\left|f_{n}(x)-f(x)\right|+\sup _{x, y \in S, x \neq y} \frac{\left|\left(f_{n}(x)-f(x)\right)-\left(f_{n}(y)-f(y)\right)\right|}{d_{S}(x, y)} \rightarrow 0 \quad$ as $n \rightarrow \infty$.

First we consider the case $f \in C^{\infty}(S, M)$. As in Lemma 4.1, we only need to prove the assertion locally in $S$. By choosing $\epsilon>0$ small if necessary, we may assume that for any $s \in S$, there exists an open neighborhood $U \subset S$ of $s$ such that $f_{\xi}(U) \subset U_{\alpha}$ for all $\xi \in \mathbb{B}_{\epsilon}^{l}$ and for some $\alpha$. As in the proof of Lemma 4.1, there exists $\left\{A_{\alpha, n}\right\} \subset C^{\infty}\left(U_{\alpha}, T^{*} U_{\alpha} \otimes \mathfrak{g}\right)$ such that $A_{\alpha, n} \rightarrow A_{\alpha}$ in $L^{p}\left(U_{\alpha}\right)$ and $d A_{\alpha, n} \rightarrow d A_{\alpha}$ in $L^{p / 2}\left(U_{\alpha}\right)$. Then as in the proof of Lemma 4.1, we have

$$
\int_{S} f_{\xi}^{*} P_{\phi}\left(A_{\alpha, n}\right) \wedge d \beta=0
$$

for any $\beta \in C^{\infty}\left(S, \bigwedge^{i-2 k-1} T^{*} S\right)$ with $\operatorname{supp}(\beta) \subset U$ and $\xi \in \mathbb{B}_{\epsilon}^{l}$.

Once we prove $f_{\xi}^{*} P_{\phi}\left(A_{\alpha, n}\right) \rightarrow f_{\xi}^{*} P_{\phi}\left(A_{\alpha}\right)$ in $L^{p / 2 k}\left(U, \bigwedge^{2 k} T^{*} U\right)$ for a.e. $\xi \in \mathbb{B}_{\epsilon}^{l}$, the assertion $d\left(f_{\xi}^{*} P_{\phi}(A)\right)=0$ in $\mathcal{D}^{\prime}\left(S, \wedge^{2 k} T^{*} S\right)$ for a.e. $\xi \in \mathbb{B}_{\epsilon}^{l}$ follows from (4.5) and the partition of unity argument. However, this is a consequence of Lemma 3.1 and the assertion follows in the case $f \in C^{\infty}(S, M)$.

Next we consider the general case $f \in \operatorname{Lip}(S, M)$. Let $\left\{f_{n}\right\} \subset C^{\infty}(S, M)$ be an approximating sequence of $f$ as in the beginning of the proof. Then $\left(f_{n}\right)_{\xi}$ approximates $f_{\xi}$ in $\operatorname{Lip}(M, S)$. From what we have proved above we have

$$
\int_{S}\left(f_{n}\right)_{\xi}^{*} P_{\phi}(A) \wedge d \beta=0
$$

for any $\beta \in C^{\infty}\left(S, \bigwedge^{i-2 k-1} T^{*} S\right)$ and for a.e. $\xi \in \mathbb{B}_{\epsilon}$. Passing to the limit $n \rightarrow \infty$ in (4.6) we obtain

$$
\int_{S} f_{\xi}^{*} P_{\phi}(A) \wedge d \beta=0
$$

for any $\beta \in C^{\infty}\left(S, \bigwedge^{i-2 k-1} T^{*} S\right)$ and a.e. $\xi \in \mathbb{B}_{\epsilon}^{l}$. This completes the proof.

Suppose as before $p \geq 2$. Let $P=\left\langle\left\{U_{\alpha},\left\{g_{\alpha \beta}\right\}\right\rangle \in \mathcal{P}_{G}^{1, p}(M)\right.$ and $A=$ $\left\{A_{\alpha}\right\} \in \mathfrak{A}^{1, p / 2}$. Also suppose $k \leq p / 2$ and $\phi \in I^{k}(G)$. Let $\sigma: \triangle_{2 k} \rightarrow$ $M$ be a smooth $2 k$-simplex, where $\triangle_{2 k}$ is the standard $2 k$-simplex $\triangle_{2 k}=$ $\left\{\sum_{i=0}^{2 k} \lambda_{i} e_{i}: \sum_{i=0}^{2 k} \lambda_{i}=1,0 \leq \lambda_{i} \leq 1\right\} \subset \mathbb{R}^{2 k+1}$ and $e_{0}=(1,0,0, \ldots, 0)$, $e_{1}=(0,1,0, \ldots, 0), \ldots, e_{2 k}=(0,0,0, \ldots, 1) \in \mathbb{R}^{2 k+1}$.

Let $P_{2 k}=\left\{\left(x_{0}, x_{1}, \ldots, x_{2 n}\right) \in \mathbb{R}^{2 k+1}: \sum_{i=0}^{2 k} x_{i}=1\right\} \subset \mathbb{R}^{2 k+1}$ be a hyperplane and $\mathcal{U}\left(\triangle_{2 k}\right) \subset P_{2 k}$ an open neighborhood of $\triangle_{2 k}$ in $P_{2 k}$. By the smoothness of $\sigma$, we may assume that $\sigma$ is extended as a smooth map on $\mathcal{U}\left(\triangle_{2 k}\right)$ which we also denote by $\sigma$. 
In the following, we want to define a $2 k$-cochain $P_{\phi}(A) \in C^{2 k}(M)=$ $\operatorname{Hom}\left(C_{2 k}(M), \mathbb{R}\right)$ which is defined on the basis $\{\sigma\}$ of $2 k$-chains $C_{2 k}(M)$ by

$$
" P_{\phi}(A)(\sigma)=\int_{\sigma} P_{\phi}(A):=\int_{\triangle_{2 k}} \sigma^{*} P_{\phi}(A) "
$$

The above definition has a problem since the $2 k$-form $\sigma^{*} P_{\phi}(A)$ is not integrable over $\triangle_{2 k}$ in general. However, by the Fubini's theorem as proved in Lemma 3.1, $P_{\phi}(A)\left(\sigma_{\xi}\right)$ has a well-defined meaning for a.e. $\xi \in \mathbb{B}_{\epsilon}$, where $\sigma_{\xi}=\iota_{\xi} \circ \sigma$. If the value $P_{\phi}(A)\left(\sigma_{\xi}\right)$ is independent of a.e. $\xi \in \mathbb{B}_{\epsilon}^{l}$, we can define $P_{\phi}(A)(\sigma)$ as this common value and obtain a cochain $P_{\phi}(A) \in$ $C^{2 k}(M)$. However, $P_{\phi}(A)(\sigma)$ does depend on the choice of $\xi$ and the above attempt does not succeed. For this reason, we do not attempt to define a cochain $P_{\phi}(A) \in C^{2 k}(M)$, but define directly a cohomology class $\left[P_{\phi}(A)\right] \in H^{2 k}(M ; \mathbb{R})$.

Thus let $S=\sum_{i=1}^{p} n_{i} \sigma_{i} \in C_{2 k}(M)$ be a smooth cycle, i.e., $n_{i} \in \mathbb{Z}$, $\sigma_{i}: \triangle_{2 k} \rightarrow M$ are smooth simplexes and $\partial S=0$. As before we assume that each $\sigma_{i}$ is smooth and defined on $\mathcal{U}\left(\triangle_{2 k}\right)$. Taking $\epsilon>0$ small if necessary, we may also assume that the $\epsilon$-neighborhood of $\triangle_{2 k}$ in $P_{2 k}$ is contained in $\mathcal{U}\left(\triangle_{2 k}\right)$.

For $\xi \in \mathbb{B}_{\epsilon}^{l}$, define $S_{\xi}=\sum_{i=1}^{p} n_{i} \sigma_{i, \xi}$, where $\sigma_{i, \xi}=\iota_{\xi} \circ \sigma_{i}$. Then $S_{\xi}$ is also a cycle: $\partial S_{\xi}=0$. By the Fubini's theorem (see Lemma 3.1), $\sigma_{i, \xi}^{*} P_{\phi}(A)$ is in $L^{1}\left(\triangle_{2 k}\right)$ for a.e. $\xi \in \mathbb{B}_{\epsilon}^{l}$ and we define

$$
P_{\phi}(A)\left(S_{\xi}\right):=\sum_{i=1}^{p} n_{i} \int_{\triangle_{2 k}} \sigma_{i, \xi}^{*} P_{\phi}(A) .
$$

Then we have:

Lemma 4.3 Let $S$ be a $2 k$-cycle as above. Then for a.e. $\xi_{0}, \xi_{1} \in \mathbb{B}_{\epsilon}^{l}$, we have $P_{\phi}(A)\left(S_{\xi_{0}}\right)=P_{\phi}(A)\left(S_{\xi_{1}}\right)$.

To prove the above lemma, we need some preparation. For $\xi, \xi_{0}, \xi_{1} \in \mathbb{B}_{\epsilon / 2}^{l}$ and a smooth simplex $\sigma: \triangle \rightarrow M$ define

$$
\begin{aligned}
i_{\xi_{0}, \xi_{1}}: M \times[-1,2] & \rightarrow M, \quad i_{\xi_{0}, \xi_{1} ; \xi}: M \times[-1,2] \rightarrow M, \\
H_{\sigma: \xi_{0}, \xi_{1}}: \triangle_{2 k} \times[-1,2] & \rightarrow M, \quad H_{\sigma: \xi_{0}, \xi_{1} ; \xi}: \triangle_{2 k} \times[-1,2] \rightarrow M
\end{aligned}
$$

by

$$
\begin{aligned}
i_{\xi_{0}, \xi_{1}}(x, t) & =\pi_{M}\left(x+(1-\varphi(t)) \xi_{0}+\varphi(t) \xi_{1}\right), \\
i_{\xi_{0}, \xi_{1} ; \xi}(x, t) & =\pi_{M}\left(x+(1-\varphi(t)) \xi_{0}+\varphi(t) \xi_{1}+\xi\right), \\
H_{\sigma: \xi_{0}, \xi_{1}}(x, t) & =i_{\xi_{0}, \xi_{1}}(\sigma(x), t), \quad H_{\sigma: \xi_{0}, \xi_{1} ; \xi}(x, t)=i_{\xi_{0}, \xi_{1} ; \xi}(\sigma(x), t),
\end{aligned}
$$

where $\varphi \in C^{\infty}(\mathbb{R})$ is such that $\varphi(t)=0$ for $t \leq 0, \varphi(t)=1$ for $t \geq 1$ and $0 \leq \varphi(t) \leq 1$ for $0<t<1$. 
Before proving Lemma 4.3, we first prove:

Lemma 4.4 For $\xi_{0}, \xi_{1} \in \mathbb{B}_{\epsilon / 2}^{l}$ and a.e. $\xi \in \mathbb{B}_{\epsilon / 2}^{l}$, we have

$$
\int_{\partial\left(\triangle_{2 k} \times[0,1]\right)} H_{\sigma: \xi_{0}, \xi_{1} ; \xi}^{*} P_{\phi}(A)=0 .
$$

Proof. We may assume, as before, that $\sigma$ is defined on $\mathcal{U}\left(\triangle_{2 k}\right)$. By Lemma 4.2, we have

$d\left(H_{\sigma: \xi_{0}, \xi_{1} ; \xi}^{*} P_{\phi}(A)\right)=0$ in $\mathcal{D}^{\prime}\left(\mathcal{U}\left(\triangle_{2 k}\right) \times(-1,2), \bigwedge^{2 k+1} T^{*}\left(\mathcal{U}\left(\triangle_{2 k}\right) \times(-1,2)\right)\right)$

for a.e. $\xi \in \mathbb{B}_{\epsilon / 2}^{l}$. That is, for any $\alpha \in C_{0}^{\infty}\left(\mathcal{U}\left(\triangle_{2 k}\right) \times(-1,2)\right)$, we have

$$
\int_{u\left(\triangle_{2 k}\right) \times(-1,2)} H_{\sigma: \xi_{0}, \xi_{1} ; \xi}^{*} P_{\phi}(A) \wedge d \alpha=0 .
$$

We denote by $c$ the barycenter of $\triangle_{2 k} \times[0,1]$ and define the radial function with center at $c$ by $|x|=\inf \left\{\lambda: \lambda>0, x \in c+\lambda\left(\triangle_{2 k} \times[0,1]-c\right)\right\}$. For $x \in \triangle_{2 k} \times[0,1]$ and $\epsilon \in(0,1)$, define a Lipschitz function $\alpha_{\epsilon}$ by setting $\alpha_{\epsilon}(x)=1$ for $|x| \leq 1-\epsilon, \alpha_{\epsilon}(x)=(1-|x|) / \epsilon$ for $1-\epsilon<|x| \leq 1$. $\alpha_{\epsilon}$ is extended to $\mathcal{U}\left(\triangle_{2 k}\right) \times(-1,2)$ by 0 as a Lipschitz function and we also denote this extension by the same notation $\alpha_{\epsilon}$. By approximating $\alpha_{\epsilon}$ by smooth functions, we see that the same equation (4.9) holds for $\alpha=\alpha_{\epsilon}$ and we therefore have:

$$
\frac{1}{\epsilon} \int_{1-\epsilon \leq|x| \leq 1} H_{\sigma: \xi_{0}, \xi_{1} ; \xi}^{*} P_{\phi}(A) \wedge d|x|=0
$$

Thus by the differentiation theorem of the Lebesgue integral and letting $\epsilon \rightarrow 0$ in (4.10), we obtain that for a.e. $\xi \in \mathbb{B}_{\epsilon / 2}^{l}$

$$
\int_{|x|=1} H_{\sigma: \xi_{0}, \xi_{1} ; \xi}^{*} P_{\phi}(A)=0 .
$$

This completes the proof of the Lemma.

Proof of Lemma 4.3. By Lemma 4.4, we have for a.e. $\xi \in \mathbb{B}_{\epsilon / 2}^{l}$

$$
\begin{aligned}
0= & \int_{\partial\left(\triangle_{2 k} \times[0,1]\right)} H_{\sigma_{i}: \xi_{0}, \xi_{1} ; \xi}^{*} P_{\phi}(A) \\
= & \int_{\partial \triangle_{2 k} \times[0,1]} H_{\sigma_{i}: \xi_{0}, \xi_{1} ; \xi}^{*} P_{\phi}(A)+\int_{\triangle_{2 k}}\left(\sigma_{i}\right)_{\xi_{1}+\xi}^{*} P_{\phi}(A) \\
& -\int_{\triangle_{2 k}}\left(\sigma_{i}\right)_{\xi_{0}+\xi}^{*} P_{\phi}(A),
\end{aligned}
$$


Since $H_{\sigma_{i}: \xi_{0}, \xi_{1} ; \xi}=i_{\xi_{0}, \xi_{1} ; \xi} \circ\left(\sigma_{i} \times\right.$ id $)($ id $:[0,1] \rightarrow[0,1]$ is the identity map) we obtain

$$
\begin{aligned}
\int_{\partial \triangle_{2 k} \times[0,1]} H_{\sigma_{i}: \xi_{0}, \xi_{1} ; \xi}^{*} P_{\phi}(A) & =\int_{\partial \triangle_{2 k} \times[0,1]}\left(\sigma_{i} \times \mathrm{id}\right)^{*} i_{\xi_{0}, \xi_{1} ; \xi}^{*} P_{\phi}(A) \\
& =\int_{\partial \triangle_{2 k}} \sigma_{i}^{*}\left(\int_{0}^{1} d t \wedge \iota_{\frac{\partial}{\partial t}}\left(i_{\xi_{0}, \xi_{1} ; \xi}^{*} P_{\phi}(A)\right)\right),
\end{aligned}
$$

where $\iota_{\frac{\partial}{\partial t}}$ denotes the contraction by $\frac{\partial}{\partial t}$.

Since $P_{\phi}(A) \in L^{1}\left(M, \bigwedge^{2 k} T^{*} M\right)$, there exists $\omega_{n} \in C^{\infty}\left(M, \bigwedge^{2 k} T^{*} M\right)$ such that $\omega_{n} \rightarrow P_{\phi}(A)$ in $L^{1}\left(M, \bigwedge^{2 k} T^{*} M\right)$. Then by Fubini's theorem, we have:

$$
\begin{aligned}
& \int_{\mathbb{B}_{\epsilon / 2}^{l}}\left(\int_{\partial \triangle_{2 k}}\right.\left.\int_{0}^{1}\left|\sigma_{i}^{*}\left(\iota_{\frac{\partial}{\partial t}}\left(i_{\xi_{0}, \xi_{1} ; \xi}^{*}\left(P_{\phi}(A)-\omega_{n}\right)\right)\right)\right|\right) \mathrm{d} \mathcal{H}^{l}(\xi) \\
& \leq C \int_{M}\left|P_{\phi}(A)-\omega_{n}\right| \operatorname{dvol}_{M} \rightarrow 0 \quad \text { as } n \rightarrow \infty,
\end{aligned}
$$

where in the above, $C>0$ is a constant independent of $\xi_{0}, \xi_{1}, \xi$ and $n$.

Therefore by (4.14), Fubini's theorem and Fatou's lemma, there exists a subsequence of $\left\{\omega_{n}\right\}$ (which we also denote by $\left\{\omega_{n}\right\}$ ) such that for a.e. $\xi \in \mathbb{B}_{\epsilon / 2}^{l}$ we obtain

$$
\int_{\partial \triangle_{2 k}} \int_{0}^{1}\left|\sigma_{i}^{*}\left(\iota_{\frac{\partial}{\partial t}}\left(i_{\xi_{0}, \xi_{1} ; \xi}^{*}\left(P_{\phi}(A)-\omega_{n}\right)\right)\right)\right| \rightarrow 0
$$

as $n \rightarrow \infty$.

Thus we have from (4.12), (4.13) and (4.15):

$$
\begin{aligned}
P_{\phi}(A)\left(S_{\xi_{1}+\xi}\right)-P_{\phi}\left(S_{\xi_{0}+\xi}\right) & =\sum_{i=1}^{p} n_{i}\left(\int_{\triangle_{2 k}} \sigma_{i, \xi_{1}+\xi}^{*} P_{\phi}(A)-\int_{\triangle_{2 k}} \sigma_{i, \xi_{0}+\xi}^{*} P_{\phi}(A)\right) \\
= & -\sum_{i=1}^{p} n_{i} \int_{\partial \triangle_{2 k} \times[0,1]} H_{\sigma_{i}: \xi_{0}, \xi_{1} ; \xi}^{*} P_{\phi}(A) \\
= & -\lim _{n \rightarrow \infty} \sum_{i=1}^{p} n_{i} \int_{\partial \triangle_{2 k}} \sigma_{i}^{*}\left(\int_{0}^{1} d t \wedge \iota_{\frac{\partial}{\partial t}}\left(i_{\xi_{0}, \xi_{1} ; \xi}^{*} \omega_{n}\right)\right)
\end{aligned}
$$

for a.e. $\xi \in \mathbb{B}_{\epsilon / 2}^{l}$.

The last term in (4.16) vanishes since $\partial S=0$. Thus we have proved

$$
P_{\phi}(A)\left(S_{\xi_{0}+\xi}\right)=P_{\phi}\left(S_{\xi_{1}+\xi}\right) \quad \text { for a.e. } \xi \in \mathbb{B}_{\epsilon / 2}^{l} \text {. }
$$

Since (4.17) holds for any $\xi_{0}, \xi_{1} \in \mathbb{B}_{\epsilon / 2}^{l}$ and a.e. $\xi \in \mathbb{B}_{\epsilon / 2}^{l}$, by the similar argument as in the proof of Lemma 3.2, we have proved the assertion of Lemma 4.3. 
By Lemma 4.3, we give the following definition:

Definition 4.1 Let $\phi \in I^{k}(G)$ and $S=\sum_{i} n_{i} \sigma_{i}$ a $2 k$-cycle on $M$. We define $P_{\phi}(A)(S)$ by

$$
P_{\phi}(A)(S)=P_{\phi}(A)\left(S_{\xi}\right)
$$

which is finite and independent of a.e. $\xi \in \mathbb{B}_{\epsilon / 2}^{l}$.

The next lemma shows that $P_{\phi}(A)$ defines an element in the cohomology group $H^{2 k}(M ; \mathbb{R})=\operatorname{Hom}\left(H_{2 k}(M ; \mathbb{R}), \mathbb{R}\right)$.

Lemma 4.5 Suppose $P=\left\langle\left\{U_{\alpha}\right\},\left\{g_{\alpha \beta}\right\}\right\rangle \in \mathcal{P}_{G}^{1, p}(M)$ and $A=\left\{A_{\alpha}\right\} \in \mathfrak{A}^{1, p / 2}(P)$ for some $p \geq 2$. Let us also assume that $\phi \in I^{k}(G)$ for some $k \leq p / 2$. Then for any smooth $(2 k+1)$-chain $S$ on $M$, we have $P_{\phi}(A)(\partial S)=0$.

Proof. It suffices to prove the assertion for smooth simplex $\sigma: \triangle_{2 k+1} \rightarrow M$. As before, we may assume that $\sigma$ is defined on $\mathcal{U}\left(\triangle_{2 k+1}\right) \rightarrow M$ and the $\epsilon$-neighborhood of $\triangle_{2 k+1}$ in $P_{2 k+1}$ is contained in $\mathcal{U}\left(\triangle_{2 k+1}\right)$. By Lemma 4.2, we have $d\left(\sigma_{\xi}^{*} P_{\phi}(A)\right)=0$ in $\mathcal{D}^{\prime}\left(\mathcal{U}\left(\triangle_{2 k+1}\right), \bigwedge^{2 k+1} T^{*} \mathfrak{U}\left(\triangle_{2 k+1}\right)\right)$ for a.e. $\xi \in \mathbb{B}_{\epsilon}^{l}$. Therefore for any $\alpha \in C_{0}^{\infty}\left(\mathcal{U}\left(\triangle_{2 k+1}\right)\right)$, we have for a.e $\xi \in \mathbb{B}_{\epsilon}^{l}$

$$
\int_{\mathcal{U}\left(\triangle_{2 k+1}\right)} \sigma_{\xi}^{*} P_{\phi}(A) \wedge d \alpha=0
$$

From this, arguing as in the proof of Lemma 4.4, we obtain

$$
\int_{\partial \triangle_{2 k+1}} \sigma_{\xi}^{*} P_{\phi}(A)=0 \quad \text { for a.e. } \xi \in \mathbb{B}_{\epsilon}^{l} .
$$

From Definition 4.1 and (4.18), we have $P_{\phi}(A)(\partial \sigma)=0$.

From Lemma 4.5, we give the following definition:

Definition 4.2 Suppose $p \geq 2, P=\left\langle\left\{U_{\alpha}\right\},\left\{g_{\alpha \beta}\right\}\right\rangle \in \mathcal{P}_{G}^{1, p}(M), A=\left\{A_{\alpha}\right\} \in$ $\mathfrak{A}^{1, p / 2}(P)$ and $\phi \in I^{k}(G)$ with $k \leq p / 2$. By Lemma 4.5, $P_{\phi}(A)$ defines a cohomology class in $H^{2 k}(M ; \mathbb{R})=\operatorname{Hom}\left(H_{2 k}(M, \mathbb{R}), \mathbb{R}\right)$ which we denote by $\left[P_{\phi}(A)\right]:\left[P_{\phi}(A)\right]([S]):=P_{\phi}(A)(S)$.

The following theorem is one of the main results of this section. It asserts that the analog of the classical Chern-Weil theory holds for $P \in \mathcal{P}_{G}^{1, p}(M)$ and connections in $\mathfrak{A}^{1, p / 2}(P)$ up to dimension $p / 2$.

Theorem 4.1 Let us assume that $p \geq 2, P=\left\langle\left\{U_{\alpha}\right\},\left\{g_{\alpha \beta}\right\}\right\rangle \in \mathcal{P}_{G}^{1, p}(M)$ and $\phi \in I^{k}(G)$ for $k \leq p / 2$. Then the cohomology class $\left[P_{\phi}(A)\right] \in H^{2 k}(M ; \mathbb{R})$ defined in Definition 4.2 is independent of $A \in \mathfrak{A}^{1, p / 2}(P)$. 
Proof. Let $A_{1}=\left\{A_{1, \alpha}\right\}$ and $A_{2}=\left\{A_{2, \alpha}\right\}$ be two connections in $\mathfrak{A}^{1, p / 2}(P)$. Denote by $\operatorname{pr}_{1}: M \times(-1,2) \rightarrow M$ the projection to the first factor. Define the pull back bundle $\operatorname{pr}_{1}^{*} P$ on $M \times(-1,2)$ by $\operatorname{pr}_{1}^{*} P=\left\langle\left\{U_{\alpha} \times(-1,2)\right\},\left\{\operatorname{pr}_{1}^{*} g_{\alpha \beta}\right\}\right\rangle$, where $\operatorname{pr}_{1}^{*} g_{\alpha \beta}(x, t)=g_{\alpha \beta}(x)$. It is easy to see that $\operatorname{pr}_{1}^{*} P$ is a principal $G$-bundle of class $W^{1, p}$ on $M \times(-1,2)$.

Let $\varphi \in C^{\infty}(\mathbb{R})$ be such that $\varphi(t)=0$ for $t \leq 0, \varphi(t)=1$ for $t \geq 1$ and $0 \leq \varphi(t) \leq 1$ for $0<t<1$. For each $\alpha$, we define

$$
A_{\alpha}(x, t)=\varphi(t) A_{1, \alpha}(x)+(1-\varphi(t)) A_{2, \alpha}(x) .
$$

It is also easy to see that $A=\left\{A_{\alpha}\right\}$ is a $\mathfrak{A}^{1, p / 2}$-connection on $\operatorname{pr}_{1}^{*} P$, i.e., it satisfies

$$
A_{\beta}=\left(\operatorname{pr}_{1}^{*} g_{\alpha \beta}\right)^{-1} d\left(\operatorname{pr}_{1}^{*} g_{\alpha \beta}\right)+\left(\operatorname{pr}_{1}^{*} g_{\alpha \beta}\right)^{-1} A_{\alpha}\left(\operatorname{pr}_{1}^{*} g_{\alpha \beta}\right)
$$

in $U_{\alpha \beta} \times(-1,2), A_{\alpha} \in L^{p}\left(U_{\alpha}\right)$ and $d A_{\alpha} \in L^{p / 2}\left(U_{\alpha}\right)$.

What we want to prove is that for any $2 k$-cycle $S$ on $M$, there holds $P_{\phi}\left(A_{1}\right)(S)=P_{\phi}\left(A_{2}\right)(S)$. As before, we may assume that $S$ is smooth and can be written as $S=\sum_{i=1}^{p} n_{i} \sigma_{i}$ for smooth simplexes $\sigma_{i}: \triangle_{2 k} \rightarrow M$ and $n_{i} \in \mathbb{Z}$.

For each simplex $\sigma_{i}$, define a $(2 k+1)$-simplex $\hat{\sigma}_{i}$ on $M \times(-1,2)$ by

$$
\hat{\sigma}_{i}: \triangle_{2 k} \times[-1 / 2,3 / 2] \rightarrow M \times(-1,2), \quad \hat{\sigma}_{i}(x, t)=\left(\sigma_{i}(x), t\right) .
$$

Also for $\xi \in \mathbb{B}_{\epsilon / 2}^{l}$ and $\tau \in(-1 / 2,1 / 2)$, define $\hat{\sigma}_{i,(\xi, \tau)}$ by

$$
\hat{\sigma}_{i,(\xi, \tau)}(x, t)=\left(\sigma_{i, \xi}(x), t+\tau\right) .
$$

Then as in the proof of Lemma 4.4, we have from Lemma 4.2

$$
\begin{aligned}
0 & =\int_{\partial\left(\triangle_{2 k} \times[-1 / 2,3 / 2]\right)} \hat{\sigma}_{i,(\xi, \tau)}^{*} P_{\phi}(A) \\
& =\int_{\partial \triangle_{2 k} \times[-1 / 2,3 / 2]} \hat{\sigma}_{i,(\xi, \tau)}^{*} P_{\phi}(A)+\int_{\triangle_{2 k}} \sigma_{i, \xi}^{*} P_{\phi}\left(A_{1}\right)-\int_{\triangle_{2 k}} \sigma_{i, \xi}^{*} P_{\phi}\left(A_{2}\right)
\end{aligned}
$$

for a.e. $(\xi, \tau)$.

Thus for a.e. $\xi \in \mathbb{B}_{\epsilon / 2}^{l}$ and a.e. $\tau \in(-1 / 2,1 / 2)$, we have from Lemma 4.3 that

$$
P_{\phi}\left(A_{1}\right)(S)-P_{\phi}\left(A_{2}\right)(S)=-\sum_{i=1}^{p} n_{i} \int_{\partial \triangle_{2 k} \times[-1 / 2,3 / 2]} \hat{\sigma}_{i,(\xi, \tau)}^{*} P_{\phi}(A) .
$$

To complete the proof, we need to show that the right side of $(4.20)$ is 0 . To see this, notice that $\hat{\sigma}_{i,(\xi, \tau)}$ is written as $\hat{\sigma}_{i,(\xi, \tau)}=\left(\operatorname{id}_{M} \times \iota_{\tau}\right) \circ\left(\sigma_{i, \xi} \times \mathrm{id}_{\mathbb{R}}\right)$, where $\operatorname{id}_{M}$ and $\operatorname{id}_{\mathbb{R}}$ are the identity maps of $M$ and $\mathbb{R}$, respectively, and $\iota_{\tau}(t)=t+\tau$. 
Thus defining a $2 k$-from on $M \times[-1 / 2,3 / 2]$ by $\omega=\left(\operatorname{id}_{M} \times \iota_{\tau}\right)^{*} P_{\phi}(A)$, we obtain

$$
\int_{\partial \triangle_{2 k} \times[-1 / 2,3 / 2]} \hat{\sigma}_{i,(\xi, \tau)}^{*} P_{\phi}(A)=\int_{\partial \triangle_{2 k} \times[-1 / 2,3 / 2]}\left(\sigma_{1, \xi} \times \operatorname{id}_{\mathbb{R}}\right)^{*} \omega .
$$

Then as in the proof of Lemma 4.3, we have

$$
\begin{aligned}
\int_{\partial \triangle_{2 k} \times[-1 / 2,3 / 2]}\left(\sigma_{i, \xi} \times \operatorname{id}_{\mathbb{R}}\right)^{*} \omega & =\int_{\partial \triangle_{2 k}} \sigma_{i, \xi}^{*}\left(\int_{-1 / 2}^{3 / 2} d t \wedge \iota_{\frac{\partial}{\partial t}} \omega\right) \\
& =\int_{\partial \triangle_{2 k}} \sigma_{i}^{*}\left(i_{\xi}^{*}\left(\int_{-1 / 2}^{3 / 2} d t \wedge \iota_{\frac{\partial}{\partial t}} \omega\right)\right)
\end{aligned}
$$

Then arguing similarly as in the proof of Lemma 4.3, i.e., approximating $(2 k-1)$-form $\int_{-1 / 2}^{3 / 2} d t \wedge \iota_{\frac{\partial}{\partial t}} \omega$ on $M$ by smooth ones $\omega_{n} \in C^{\infty}\left(M, \bigwedge^{2 k-1} T^{*} M\right)$ in $L^{1}(M)$, we obtain by applying Fubini's theorem and Fatou's lemma that for a.e. $\xi \in \mathbb{B}_{\epsilon / 2}^{l}$ and for some subsequence of $\left\{\omega_{n}\right\}$ (which we still denote by $\left.\left\{\omega_{n}\right\}\right)$ :

$$
\int_{\partial \triangle_{2 k}} \sigma_{i}^{*}\left(i_{\xi}^{*}\left(\int_{-1 / 2}^{3 / 2} d t \wedge \iota_{\frac{\partial}{\partial t}} \omega\right)\right)=\lim _{n \rightarrow \infty} \int_{\partial \triangle_{2 k}} \sigma_{i}^{*} i_{\xi}^{*} \omega_{n} .
$$

Therefore for a.e. $\xi \in \mathbb{B}_{\epsilon / 2}^{l}$, the right side of (4.20) becomes

$$
\begin{aligned}
-\sum_{i=1}^{p} n_{i} \int_{\partial \triangle_{2 k} \times[-1 / 2,3 / 2]} \hat{\sigma}_{i,(\xi, \tau)}^{*} P_{\phi}(A) & =-\sum_{i=1}^{p} n_{i} \int_{\partial \triangle_{2 k}} \sigma_{i}^{*}\left(i_{\xi}^{*}\left(\int_{-1 / 2}^{3 / 2} d t \wedge \iota_{\frac{\partial}{\partial t}} \omega\right)\right) \\
& =-\lim _{n \rightarrow \infty} \sum_{i=1}^{p} n_{i} \int_{\partial \triangle_{2 k}} \sigma_{i}^{*} i_{\xi}^{*} \omega_{n}=0 .
\end{aligned}
$$

This completes the proof.

In view of the above, we give

Definition 4.3 For $P \in \mathcal{P}_{G}^{1, p}(M)$ and $\phi \in I^{k}(G)(k \leq p / 2)$, we define a cohomology class $P_{\phi}(P) \in H^{2 k}(M ; \mathbb{R})$ as the class $\left[P_{\phi}(A)\right] \in H^{2 k}(M ; \mathbb{R})$ which does not depend on $A \in \mathfrak{A}^{1, p / 2}(P)$.

In the above, we have defined from $\phi \in I^{k}(G)$ and $P \in \mathcal{P}_{G}^{1, p}(M)$ the cohomology class $\left[P_{\phi}(P)\right] \in H^{2 k}(M ; \mathbb{R})$. On the other hand, $P_{\phi}(A)$ for $A \in \mathfrak{A}^{1, p / 2}(P)$ naturally defines an $(m-2 k)$-(normal) current which also defines a homology class in $H_{m-2 k}(M ; \mathbb{R})$. For the smooth case, these classes are identified through Poincaré duality. We shall show below that this is also the case for $P \in \mathcal{P}_{G}^{1, p}(P)$ and $\phi \in I^{k}(G)$ when $k \leq p / 2$. 
As before, let us assume that $P \in \mathcal{P}_{G}^{1, p}(M)$ and $\phi \in I^{k}(G)$. For $A \in$ $\mathfrak{A}^{1, p / 2}(P)$, define a $(m-2 k)$-current $C_{\phi}(A)$ as

$$
C_{\phi}(A): C^{\infty}\left(M, \bigwedge^{m-2 k} T^{*} M\right) \ni \alpha \mapsto \int_{M} \alpha \wedge P_{\phi}(A) \in \mathbb{R} .
$$

The current $C_{\phi}(A)$ has the following properties:

i) The mass of $C_{\phi}(A)$ is finite:

$$
\begin{aligned}
& \begin{aligned}
M\left(C_{\phi}(A)\right) & :=\sup \left\{C_{\phi}(A)(\alpha): \alpha \in C^{\infty}\left(M, \bigwedge^{m-2 k} T^{*} M\right),|\alpha| \leq 1\right\} \\
& \leq \int_{M}\left|P_{\phi}(A)\right| \mathrm{dvol}_{M}<+\infty,
\end{aligned} \\
& \text { since }\left|P_{\phi}(A)\right| \leq C\left|F_{A}\right|^{k} \in L^{1}(M) .
\end{aligned}
$$

ii) $C_{\phi}(A)$ is a cycle:

$$
\partial C_{\phi}(A)(\alpha)=C_{\phi}(A)(d \alpha)=\int_{M} d \alpha \wedge P_{\phi}(A)=0
$$

for any $\alpha \in C^{\infty}\left(M, \bigwedge^{m-2 k} T^{*} M\right)$ by Lemma 4.1 .

Recall that a current $T$ is called normal if the masses of $T$ and $\partial T$ are finite (see [8]). From i) and ii), $C_{\phi}(A)$ defines an $(m-2 k)$-normal current, $C_{\phi}(A) \in N_{m-2 k}(M)$. From the sets $Z_{i}(M):=\left\{T \in N_{i}(M): \partial T=0\right\}$ of cycles and boundaries $B_{i}(T):=\left\{\partial T: T \in N_{i+1}(N)\right\}$, one obtains the quotient $Z_{i}(M) / B_{i}(M)$ which is defined as the $i$-th homology group of $M$ with coefficient in $\mathbb{R}: H_{i}(M ; \mathbb{R})=Z_{i}(M) / B_{i}(M)$. It is known that $H_{i}(M ; \mathbb{R})$ is isomorphic to the $i$-th singular homology group of $M$, see [8], [9]. From i) and ii $), C_{\phi}(A)$ defines the homology class in $H_{m-2 k}(M ; \mathbb{R})$ which we denote by $\left[C_{\phi}(A)\right]$.

There is an isomorphism between $H_{d R}^{i}(M ; \mathbb{R})$ and $H_{m-i}(M ; \mathbb{R})$ called the Poincaré duality:

$$
P D: H_{d R}^{i}(M ; \mathbb{R}) \ni \alpha \stackrel{\sim}{\longrightarrow} \int_{M} \bullet \wedge \alpha \in H_{m-i}(M ; \mathbb{R}) .
$$

On the other hand, there is also an isomorphism $H_{d R}^{i}(M ; \mathbb{R}) \simeq H^{i}(M ; \mathbb{R})$ $\left(H^{i}(M ; \mathbb{R})\right.$ is the $i$-th singular cohomology of $M$ with coefficient in $\left.\mathbb{R}\right)$ called the de Rham isomorphism which is induced by the map

$$
\Psi: C^{\infty}\left(M, \bigwedge^{i} T^{*} M\right) \ni \alpha \mapsto \Psi(\alpha) \in \operatorname{Hom}\left(C_{i}, \mathbb{R}\right)
$$

defined by

$$
\Psi(\alpha)(\sigma)=\int_{\sigma} \alpha:=\int_{\triangle_{i}} \sigma^{*} \alpha
$$

for smooth simplex $\sigma: \triangle_{i} \rightarrow M$. 
We show that $\left[P_{\phi}(A)\right]$ corresponds to $\left[C_{\phi}(A)\right]$ under these isomorphisms. Namely, we have:

Proposition 4.1 Under the above notations, we have

$$
P D \circ \Psi^{-1}\left(\left[P_{\phi}(A)\right]\right)=\left[C_{\phi}(A)\right] .
$$

Proof. For $\left[P_{\phi}(A)\right] \in H^{2 k}(M ; \mathbb{R})$, we first seek $\Psi^{-1}\left(\left[P_{\phi}(A)\right]\right) \in H_{d R}^{2 k}(M)$. Let us assume for the moment $k<p / 2$. Recall that $\left[P_{\phi}(A)\right]$ is defined in Definition 4.1. Suppose $\sigma: \triangle_{2 k} \rightarrow M$ is a smooth $2 k$-simplex. Since $d P_{\phi}(A)=0$ and $P_{\phi}(A) \in L^{p / 2 k}$ with $p / 2 k>1$, we have the Hodge decomposition $P_{\phi}(A)=h(A)+d \omega(A)$, where $h(A)$ is harmonic and $C^{\infty}$ and $\omega(A) \in$ $W^{1, p / 2 k}\left(M, \bigwedge^{2 k-1} T^{*} M\right)$. Thus we have

$$
P_{\phi}(A)\left(\sigma_{\xi}\right)=\int_{\triangle_{2 k}} \sigma_{\xi}^{*} P_{\phi}(A)=\int_{\triangle_{2 k}} \sigma_{\xi}^{*} h(A)+\int_{\triangle_{2 k}} \sigma_{\xi}^{*}(d \omega(A))
$$

which has a well-defined meaning for a.e. $\xi \in \mathbb{B}_{\epsilon}^{l}$.

We consider the second term in (4.26). Choose a sequence $\left\{\omega_{n}\right\} \in$ $C^{\infty}\left(M, \bigwedge^{2 k-1} T^{*} M\right)$ such that $\omega_{n} \rightarrow \omega(A)$ in $W^{1, p / 2 k}(M)$. Then by Fubini's theorem (as in Lemma 3.1) and Fatou's Lemma, there exists a subsequence of $\left\{\omega_{n}\right\}$ (still denoted by $\left\{\omega_{n}\right\}$ ) such that

$$
\int_{\triangle_{2 k}} d\left(\sigma_{\xi}^{*} \omega_{n}\right)=\int_{\triangle_{2 k}} \sigma_{\xi}^{*} d \omega_{n} \rightarrow \int_{\triangle_{2 k}} \sigma_{\xi}^{*} d \omega(A) \text { as } n \rightarrow \infty \text { for a.e. } \xi \in \mathbb{B}_{\epsilon}^{l} \text {. }
$$

Similarly, we also have

$$
\int_{\triangle_{2 k}} \sigma_{\xi}^{*} d \omega_{n}=\int_{\partial \triangle_{2 k}} \sigma_{\xi}^{*} \omega_{n} \rightarrow \int_{\partial \triangle_{2 k}} \sigma_{\xi}^{*} \omega(A) \text { as } n \rightarrow \infty \text { for a.e. } \xi \in \mathbb{B}_{\epsilon} .
$$

From (4.27) and (4.28), we obtain

$$
\int_{\triangle_{2 k}} \sigma_{\xi}^{*} d \omega(A)=\int_{\partial \triangle_{2 k}} \sigma_{\xi}^{*} \omega(A) \quad \text { for a.e. } \xi \in \mathbb{B}_{\epsilon}^{l} .
$$

By (4.26) and (4.29), we have for a.e. $\xi \in \mathbb{B}_{\epsilon}^{l}$

$$
P_{\phi}(A)\left(\sigma_{\xi}\right)=\Psi(h(A))\left(\sigma_{\xi}\right)+\int_{\partial \triangle_{2 k}} \sigma_{\xi}^{*} \omega(A) .
$$

Therefore if $S=\sum_{i=1}^{p} n_{i} \sigma_{i}$ is a cycle, we obtain

$$
\begin{aligned}
P_{\phi}(A)(S) & =P_{\phi}(A)\left(S_{\xi}\right) \quad\left(\text { for a.e } \xi \in \mathbb{B}_{\epsilon}^{l} \text {, see Definition } 4.1\right) \\
& =\Psi(h(A))\left(S_{\xi}\right) \\
& =\Psi(h(A))(S) \quad\left(\text { since } S_{\xi} \text { is homologous to } S\right) .
\end{aligned}
$$

Thus we have $\Psi([h(A)])=P_{\phi}(A)$ in $\operatorname{Hom}\left(H_{2 k}(M ; \mathbb{R}), \mathbb{R}\right)=H^{2 k}(M ; \mathbb{R})$. 
On the other hand, we have

$$
\begin{aligned}
C_{\phi}(A)(\alpha) & =\int_{M} \alpha \wedge P_{\phi}(A)=\int_{M} \alpha \wedge h(A)+\int_{M} \alpha \wedge d \omega(A) \\
& =\int_{M} \alpha \wedge h(A)+(-1)^{m(2 k-1)+1} \int_{M} d \alpha \wedge \omega(A) \\
& =P D(h(A))(\alpha)+(-1)^{m(2 k-1)+1} \partial P D(\omega(A))(\alpha) .
\end{aligned}
$$

In (4.32), $P D(\omega(A))$ is a normal $(m-2 k+1)$-current since $M(P D(\omega(A))) \leq$ $\|\omega(A)\|_{L^{1}(M)}<\infty$ and $M(\partial P D(\omega(A))) \leq\|d \omega(A)\|_{L^{1}(M)}<\infty$. From this we obtain $C_{\phi}(A)=P D(h(A))+(-1)^{m(2 k-1)+1} \partial P D(\omega(A))$ and

$$
\left[C_{\phi}(A)\right]=P D(h(A)) .
$$

Combining (4.31) and (4.33), we complete the proof for the case $k<p / 2$.

For the case $k=p / 2$, we need a deep result by Lanzani-Stein [16], see also [3], [4]. In this case the above argument should be modified since $P_{\phi}(A)$ is only in $L^{1}(M)$ and the standard Hodge decomposition theorem does not hold in this case. In this case, we apply the result of Lanzani-Stein [16] which asserts that for $q$-form $u$ satisfying $d u=f \in L^{1}$ and $d^{*} u=g \in L^{1}$, we can conclude $u \in L^{m / m-1}(M)$ provided that i) $q \neq 1, m-1$ or ii) $q=1$ and $g \in H^{1}$ ( $H^{1}$ is the Hardy space) or iii) $q=m-1$ and $f \in H^{1}$. In our case $\omega(A)$ (which exists primarily as a current) satisfies $d \omega(A) \in L^{1}(A)$, $d^{*} \omega(A)=0$ and $\omega(A)$ is a $2 k-1=p-1<m-1$ form. Thus by the result of Lanzani-Stein, we conclude $\omega(A) \in L^{m / m-1}(M)$. Once this is shown, the remaining argument is the same by approximating $\omega(A)$ by smooth forms $\omega_{n}$ such that $\omega_{n} \rightarrow \omega(A)$ in $L^{m / m-1}(M)$ and $d \omega_{n} \rightarrow d \omega(A)$ in $L^{1}(M)$. This completes the proof.

Since the cohomology class $\left[P_{\phi}(A)\right]$ is independent of $A$, we have as a corollary of Proposition 4.1 the following:

Corollary 4.1 The homology class $\left[C_{\phi}(A)\right] \in H_{m-2 k}(M ; \mathbb{R})$ is independent of $A \in \mathcal{A}^{1, p / 2}(P)$. We denote this class by $C_{\phi}(P)$. We then have $P D \circ$ $\Psi^{-1}\left(P_{\phi}(P)\right)=C_{\phi}(P)$.

The cohomology class $P_{\phi}(P)$ (and therefore $C_{\phi}(P)$ ) has some stability property which will be useful for problems in the calculus of variations. To be more precise, let us assume that there exist a sequence $\left\{P_{n}\right\}$ of bundles in the class $\mathcal{P}_{G}^{1, p}(M)$ and a sequence of connections $\left\{A_{n}\right\}$ such that $A_{n} \in \mathfrak{A}^{1, p / 2}\left(P_{n}\right)$ for all $n$ and $\sup _{n \geq 1} \int_{M}\left|F_{A_{n}}\right|^{p / 2} \operatorname{dvol}_{M}<+\infty$. Then we have:

Proposition 4.2 Let $\phi \in I^{k}(G)$. Assume that $k<p / 2$. Under the above assumption, there exists a subsequence $\left\{P_{n_{k}}\right\}$ of $\left\{P_{n}\right\}$ such that $P_{\phi}\left(P_{n_{k}}\right)$ (and $\left.C_{\phi}\left(P_{n_{k}}\right)\right)$ is independent of $k$. 
Remark 4.1 In the above proposition, the assertion does not hold for the case $k=p / 2$. This is because a phenomenon known as bubbling off of instantons occurs in general. Also one needs to take a subsequence in the above proposition in general. For example, let $P_{1}, P_{2} \in \mathcal{P}_{G}^{1, p}(M)$ and $A_{i} \in$ $\mathfrak{A}^{1, p / 2}\left(P_{i}\right)(i=1,2)$ be given such that $P_{\phi}\left(P_{1}\right) \neq P_{\phi}\left(P_{2}\right)$. Consider sequences of bundles $\left\{P_{n}\right\}$ and connections $\left\{A_{n}\right\}$ such that $P_{n}=P_{1}$ and $A_{n}=A_{1}$ if $n$ is odd and $P_{n}=P_{2}$ and $A_{n}=A_{2}$ if $n$ is even. Then the assumption of the proposition is satisfied but we have $P_{\phi}\left(P_{n}\right) \neq P_{\phi}\left(P_{n+1}\right)$ for all $n$.

Proof of Proposition 4.2. By the result of Thom [35], we have $H_{*}(M ; \mathbb{Q}) \cong$ $\Omega_{*}(M) \otimes \mathbb{Q}$, where $\Omega_{*}(M)$ is the oriented bordism ring of $M$. Thus for any $S \in H_{*}(M ; \mathbb{Z})$, there exists $k \in \mathbb{Z}$ such that $k S$ can be represented by a closed oriented submanifold of $M$. In particular, there exists a basis of $H_{*}(M ; \mathbb{Q})$ (and hence of $H_{*}(M ; \mathbb{R})$ ) consisting of the fundamental classes of closed oriented submanifolds of $M$. Thus to prove the proposition, we only need to show that $P_{\phi}\left(P_{n_{k}}\right)(S)$ is independent of $k$ for some subsequence $\left\{P_{n_{k}}\right\}$, where $S$ is a fundamental class of a closed oriented submanifold of $M$.

Assume that $S \subset M$ is a closed oriented submanifold with $\operatorname{dim} S=2 k$. By Fubini's theorem as in Lemma 3.1, we have:

$$
\begin{aligned}
\int_{\mathbb{B}_{\epsilon}^{l}}\left(\int_{S}\left|F_{A_{n}}\right|^{p / 2}\left(\pi_{M}(x+\xi)\right) \mathrm{d} \mathcal{H}^{2 k}(x)\right) \mathrm{d} \mathcal{H}^{l}(\xi) \\
\quad=\int_{S}\left(\int_{\mathbb{B}_{\epsilon}^{l}}\left|F_{A_{n}}\right|^{p / 2}\left(\pi_{M}(x+\xi)\right) \mathrm{d} \mathcal{H}^{l}(\xi)\right) \mathrm{d} \mathcal{H}^{2 k}(x) \\
\leq C \int_{S}\left(\int_{\mathcal{O}_{\epsilon}(M)}\left|F_{A_{n}}\right|^{p / 2}\left(\pi_{M}(y)\right) \mathrm{d} \mathcal{H}^{l}(y)\right) \mathrm{d} \mathcal{H}^{2 k}(x) \\
\leq C \int_{M}\left|F_{A_{n}}\right|^{p / 2} \operatorname{dvol}_{M} .
\end{aligned}
$$

By (4.34), Fubini's theorem and Fatou's lemma, for a.e. $\xi \in \mathbb{B}_{\epsilon}^{l}$, there exists a subsequence $\left\{n_{k}\right\}$ of $\{n\}$ such that

$$
\sup _{k \geq 1} \int_{S}\left|F_{A_{n_{k}}}\right|^{p / 2}\left(\pi_{M}(x+\xi)\right) \mathrm{d} \mathcal{H}^{2 k}(x)<+\infty
$$

and therefore

$$
\sup _{k \geq 1} \int_{S_{\xi}}\left|F_{A_{n_{k}}}\right|^{p / 2} \mathrm{~d} \mathcal{H}^{2 k}(x)<+\infty .
$$

From (4.35) and Uhlenbeck's weak compactness theorem [38], there exist local trivializations of the bundles $\left.P_{n_{k}}\right|_{S_{\xi}} \rightarrow S_{\xi}(k=1,2, \ldots)$ such that the connections $\left\{A_{n_{k}}\right\}$ with respect to these local trivializations are $\mathcal{A}^{1, p / 2}\left(\left.P_{n_{k}}\right|_{S_{\xi}}\right)$ bounded. From this bound, as usual, we obtain a $W^{1, p}$-bound of the gluing 
cocycles of the bundles $P_{n_{k}}$. Since $W^{1, p}$ is compactly embedded in $C^{0}$ in dimension $2 k<p$, there exists a further subsequence of $\left\{n_{k}\right\}$ (still denoted by $\left.\left\{n_{k}\right\}\right)$ such that the gluing cocycles of $P_{n_{k}}$ uniformly converge as $k \rightarrow \infty$. Thus for large $k$, the isomorphism classes of $P_{n_{k}}$ are independent of $k$. In particular, $P_{\phi}\left(P_{n_{k}}\right)\left(S_{\xi}\right)$ is independent of large $k$. Since $S_{\xi}$ is homologous to $S$, we have $P_{\phi}\left(P_{n_{k}}\right)\left(S_{\xi}\right)=P_{\phi}\left(P_{n_{k}}\right)(S)$ and this completes the proof.

Remark 4.2 It seems something strange that the class $P_{\phi}(P)$ is well-defined for $\phi \in I^{k}(G)$ up to $k=p / 2$. That is, it defines a well-defined topological invariant up to dimension p. Our theory of Sobolev bundles is closely related to the analogous theory of Sobolev mappings developed by White [41], [42]. In the theory of Sobolev mappings, one can only associate well-defined topological invariants for maps in the class $W^{1, p}$ up to dimension $p-1$. This difference probably comes from our definition of the class of Sobolev bundles. As we shall see in the next section, if we slightly relax the definition of Sobolev bundles (i.e., the classes of singular Sobolev bundles which permit certain dimensional singularities), we arrive at the similar result obtained for Sobolev mappings. The classes of singular Sobolev bundles are natural for applications, but our original classes of Sobolev bundles are also natural from the theoretical point of view. In view of Theorem 4.1, it is likely that Theorem 3.1 holds for $h: K \rightarrow M$ with $\operatorname{dim} K \leq p$. But we have not succeeded in proving or disproving such a result.

\section{Singular Sobolev bundles and connections}

In this section, we generalize the results proved in $\S 3$ and $\S 4$ for Sobolev bundles with singularities.

\subsection{Singular Sobolev bundles}

We first define classes of singular Sobolev bundles and isomorphisms between them. As before, we assume that $M$ is a compact manifold with $\operatorname{dim} M=m$.

Definition 5.1 Let $k \in \mathbb{N}, p \geq 1$ and $S \subset M$ a closed subset with $\mathcal{H}$ $\operatorname{dim} S=m-s$, where $\mathcal{H}-\operatorname{dim} S$ denotes the Hausdorff dimension of $S$. We say that $P=\left\langle\left\{U_{\alpha}\right\}_{\alpha \in I},\left\{g_{\alpha \beta}\right\}_{\alpha, \beta \in I}\right\rangle$ belongs to the class of $W^{k, p}$-Sobolev bundles over $M$ with singular set $S$ if the following holds:

i) $\left\{U_{\alpha}\right\}_{\alpha \in I}$ is an open covering of $M \backslash S$,

ii) $g_{\alpha \beta} \in W^{k, p}\left(U_{\alpha \beta}, G\right)$ for all $\alpha, \beta \in I$ with $U_{\alpha \beta} \neq \emptyset$ and $g_{\alpha \beta}(x) \cdot g_{\beta \gamma}(x)=$ $g_{\alpha \gamma}(x)$ for a.e. $x \in U_{\alpha \beta \gamma}$ whenever $U_{\alpha \beta \gamma} \neq \emptyset$.

We denote such a class of bundles by $\mathcal{P}_{G}^{k, p}(M ; S)$. 
Definition 5.2 Let $P=\left\langle\left\{U_{\alpha}\right\}_{\alpha \in I},\left\{g_{\alpha \beta}\right\}_{\alpha, \beta \in I}\right\rangle, Q=\left\langle\left\{V_{j}\right\}_{j \in J},\left\{h_{j k}\right\}_{j, k \in J}\right\rangle \in$ $\mathcal{P}_{G}^{k, p}(M ; S) . P$ and $Q$ are $W^{k, p}$-isomorphic to each other if and only if there exists a refinement $\left\{W_{s}\right\}_{s \in K}$ of both $\left\{U_{\alpha}\right\}$ and $\left\{V_{j}\right\}$, i.e., $W_{s} \subset U_{\varphi(s)}$ and $W_{s} \subset V_{\psi(s)}$ for some $\varphi: K \rightarrow I$ and $\psi: K \rightarrow J$ and $\bigcup_{s \in K} W_{s}=M \backslash S$, and a family of functions $\rho_{s} \in W^{k, p}\left(W_{s}, G\right)(s \in K)$ such that

$$
g_{\varphi(s) \varphi(t)}=\rho_{s} \cdot h_{\psi(s) \psi(t)} \cdot \rho_{t}^{-1}
$$

in $W_{\text {st }}$ for $s, t \in K$ whenever $W_{\text {st }} \neq \emptyset$.

As in the ordinary case, the isomorphism class containing $P \in \mathcal{P}_{G}^{k, p}(M ; S)$ is denoted by $[P]_{k, p}$. The set of all $W^{k, p}$-isomorphism classes is denoted by $\hat{\mathcal{P}}_{G}^{k, p}(M ; S)$.

Such classes of bundles arise naturally as limits of smooth bundles. For example, consider a sequence of Yang-Mills connections $\left\{A_{n}\right\} \in \mathcal{A}^{\infty}\left(P_{n}\right)$ on smooth bundles $P_{n} \rightarrow M$ such that $\sup _{n \geq 1} \int_{M}\left|F_{A_{n}}\right|^{2} \operatorname{dvol}_{M}<+\infty$. It was shown in [21] (see also [36]) that there exist a subsequence of $\left\{A_{n}\right\}$ (still denoted by $\left\{A_{n}\right\}$ ) and a closed set $S \subset M$ with $\mathcal{H}$-dim $S \leq m-4$ such that $\left\{A_{n}\right\}$ converges in $C_{\text {loc }}^{\infty}(M \backslash S)$ to a connection $A_{\infty}$ on a bundle $P_{\infty} \rightarrow M \backslash S$. More general result is known by the works of [20] and [30], see also $\S 7$. Namely, they proved a similar compactness theorem under the assumption of the monotonicity of the Yang-Mills energy and some approximability condition. From these, it is also useful for applications to introduce the following classes of Sobolev bundles with singularities.

Definition 5.3 Let $k \in \mathbb{N}, p \geq 1$ and $S \subset M$ be a closed subset.

i) $\mathcal{P}_{\text {strong,G }}^{k, p}(M ; S) \subset \mathcal{P}_{G}^{k, p}(M ; S)$ is defined as: $P=\left\langle\left\{U_{\alpha}\right\}_{\alpha \in I},\left\{g_{\alpha \beta}\right\}_{\alpha, \beta \in I}\right\rangle \in$ $\mathcal{P}_{G}^{k, p}(M ; S)$ belongs to $\mathcal{P}_{\text {strong, },}^{k, p}(M ; S)$ if and only if for any compact set $K \subset M \backslash S$, there exists a sequence of smooth principal $G$-bundles $\left\{P_{n}\right\} \subset \mathcal{P}_{G}^{\infty}(M)$ of the form $P_{n}=\left\langle\left\{V_{i}\right\}_{i \in J},\left\{g_{i j}^{n}\right\}_{i, j \in J}\right\rangle$ such that $\left\{V_{i} \cap\right.$ $K\}_{i \in J}$ is a refinement of $\left\{U_{\alpha} \cap K\right\}_{\alpha \in I}, V_{i} \cap K \subset U_{\varphi(i)} \cap K(\varphi: J \rightarrow I$ is a refinement map) and $\left\|g_{\varphi(i) \varphi(j)}-g_{i j}^{n}\right\|_{W^{k, p}\left(V_{i j} \cap K\right)} \rightarrow 0(n \rightarrow \infty)$ for $i, j \in J$ with $V_{i j} \neq \emptyset$.

ii) $\mathcal{P}_{\text {weak,G }}^{k, p}(M ; S) \subset \mathcal{P}_{G}^{k, p}(M ; S)$ is defined as: $P=\left\langle\left\{U_{\alpha}\right\}_{\alpha \in I},\left\{g_{\alpha \beta}\right\}_{\alpha, \beta \in I}\right\rangle \in$ $\mathcal{P}_{G}^{k, p}(M ; S)$ belongs to $\mathcal{P}_{\text {weak, },}^{k, p}(M ; S)$ if and only if for any compact set $K \subset M \backslash S$, there exists a sequence $\left\{P_{n}\right\} \subset \mathcal{P}_{G}^{\infty}(M)$ of the form $P_{n}=$ $\left\langle\left\{V_{i}\right\}_{i \in J},\left\{g_{i j}^{n}\right\}_{i, j \in J}\right\rangle$ such that $\left\{V_{i} \cap K\right\}_{i \in J}$ is a refinement of $\left\{U_{\alpha} \cap K\right\}_{\alpha \in I}$, $V_{i} \cap K \subset U_{\varphi(i)} \cap K$, and $\left\|g_{\varphi(i) \varphi(j)}-g_{i j}^{n}\right\|_{L^{p}\left(V_{i j} \cap K\right)} \rightarrow 0(n \rightarrow \infty)$ and $\sup _{n} \sum_{j=1}^{k}\left\|\nabla^{j} g_{i j}^{n}\right\|_{L^{p}\left(V_{i j} \cap K\right)}<+\infty$ for $i, j \in J$ with $V_{i j} \neq \emptyset$, where $\nabla^{j}=\nabla \cdots \nabla$ (j-times).

In the following, we generalize Theorems 3.1, 3.2 and 3.3 for bundles in the classes $\mathcal{P}_{G}^{k, p}(M ; S), \mathcal{P}_{\text {strong, } G}^{k, p}(M ; S)$ and $\mathcal{P}_{\text {weak }, G}^{k, p}(M ; S)$, respectively. 
It is straightforward to show that all these results are extendible if we replace $h: K \rightarrow M$ by $h: K \rightarrow M \backslash S$. But what we really want to know is how naturally defined invariants like in Theorem 3.1, Theorem 3.2 and Theorem 3.3 are preserved under convergence, so it is necessary to see how we can define invariants like in these theorems for bundles with singularities. That is to say, for bundles with singularities we need to see whether we can define homotopy invariant for $h: K \rightarrow M$.

Let $K$ be a $d$-dimensional polyhedron and $S \subset M$ a closed set with $\mathcal{H}-\operatorname{dim} S=m-s$ for some $0 \leq s \leq m$. To define a homotopy invariant for a Sobolev bundle with singular set $S$ and $h: K \rightarrow M$, we need to know how $h(K)$ intersects with $S$. If $h(K)$ intersects with $S$ for generic $h$, we can not define a homotopy invariant for such a pair. The following lemma gives a condition under which $h(K) \cap S=\emptyset$ holds.

Lemma 5.1 Let $K$ be a d-dimensional polyhedron and $h: K \rightarrow M$ a piecewise smooth map, i.e., its restriction to each cell of $K$ is smooth. Suppose $S \subset M$ is a closed subset with $\mathcal{H}$-dim $S=m-s$ for some $0 \leq s \leq m$ and has a $\sigma$-finite $\mathcal{H}^{m-s}$-measure, i.e., there exist closed subsets $S_{i} \subset M$ with $S=\bigcup_{i=1}^{\infty} S_{i}$ and $\mathcal{H}^{m-s}\left(S_{i}\right)<+\infty$. If $d<s$, then for $\mathcal{H}^{2 l}$-a.e. $(u, v) \in \mathbb{B}_{\epsilon}^{l} \times \mathbb{B}_{\epsilon}^{l}$, we have $h_{u, v}(K) \cap S=\emptyset$, where $h_{u, v}: K \rightarrow M$ is defined by $h_{u, v}(x)=\iota_{u}^{-1} \circ h_{v}(x)$.

For smooth $K$ and $S$, the above lemma is a simple consequence of the transversality theorem. For the general case, the proof is somewhat involved. The proof is given in the appendix.

Using Lemma 5.1, we can prove extensions of Theorems 3.1, 3.2 and 3.3. The first is the extension of Theorem 3.1.

Theorem 5.1 Let $S \subset M$ be a closed set with $\mathcal{H}-\operatorname{dim} S=m-s$ for some $0 \leq s \leq m$ and has a $\sigma$-finite $\mathcal{H}^{m-s}$-measure and $P \in \mathcal{P}_{G}^{k, p}(M ; S)$. Suppose $K$ is a d-dimensional polyhedron satisfying (A-1), (A-2) in the appendix. Under the assumption $d \leq \min \{s-2,[k p]-1\}$, for any Lipschitz map $h: K \rightarrow M$ we can associate the class $[P]_{h, K} \in \hat{\mathcal{P}}_{G}^{0}(K)$ which depends only on the homotopy class of $h$ and the $W^{k, p}$-isomorphism class of $P$. If $3 \leq k p<4$, the same result holds for $d \leq \min \{s-2,3\}$.

Proof. By the Gagliardo-Nirenberg inequality as before, we have $P \in$ $\mathcal{P}_{G}^{1, k p}(M ; S)$. The idea of the proof is essentially the same as that of the proof of Theorem 3.1 once we have Lemma 5.1. We only sketch the argument here and leave the details to the reader. We first assume that $h: K \rightarrow M$ is piecewise smooth. As in Theorem 3.1, the class $\left[h^{*} P\right]_{0}$ should be defined as the $C^{0}$-isomorphism class of $h_{u, v}^{*} P$ for $\mathcal{H}^{2 l}$-a.e. $(u, v) \in \mathbb{B}_{\epsilon}^{l} \times \mathbb{B}_{\epsilon}^{l}$. 
By the Fubini type Lemma 3.1, Lemma 5.1 and the Sobolev embedding, for $\mathcal{H}^{2 l}$-a.e. $(u, v) \in \mathbb{B}_{\epsilon}^{l} \times \mathbb{B}_{\epsilon}^{l}$ we have $h_{u, v}^{*} P:=\left\langle\left\{h_{u, v}^{-1}\left(U_{\alpha}\right)\right\},\left\{g_{\alpha \beta} \circ h_{u, v}\right\}\right\rangle \in$ $\mathcal{P}_{G}^{1, k p}(K) \subset \mathcal{P}_{G}^{0}(K)$. We need to show that their isomorphism classes are independent of $\mathcal{H}^{2 l}$-a.e. $(u, v) \in \mathbb{B}_{\epsilon / 3}^{l} \times \mathbb{B}_{\epsilon}^{l}$ and depends only on the $W^{k, p_{-}}$ isomorphism class and the homotopy class of $h$. To prove the independence of the class $\left[h_{u, v}^{*} P\right]_{0}$ for $\mathcal{H}^{2 l}$-a.e. $(u, v) \in \mathbb{B}_{\epsilon / 3}^{l} \times \mathbb{B}_{\epsilon / 3}^{l}$, we proceed as in the proof of Lemma 3.2. In this case, for any fixed $(u, v) \in \mathbb{B}_{2 \epsilon / 3}^{l} \times \mathbb{B}_{2 \epsilon / 3}^{l}$ and $\left(u^{\prime}, v^{\prime}\right) \in \mathbb{B}_{\epsilon / 3}^{l} \times \mathbb{B}_{\epsilon / 3}^{l}$, we define $H_{u^{\prime}, v^{\prime}}(x, t)=\iota_{\varphi(t) u+u^{\prime}}^{-1} \circ h_{\varphi(t) v+v^{\prime}}(x)$, where the cut-off function $\varphi$ is defined as in the proof of Lemma 3.2. Then by slightly modifying the argument of the proof of Lemma 5.1, we see that for $\mathcal{H}^{2 l}$-a.e. $\left(u^{\prime}, v^{\prime}\right) \in \mathbb{B}_{\epsilon / 3}^{l} \times \mathbb{B}_{\epsilon / 3}^{l}$ we have $H_{u^{\prime}, v^{\prime}}([-1,2] \times K) \cap S=\emptyset$ under the assumption $d \leq \min \{s-2,[k p]-1\}$. Then arguing exactly as in the proof of Lemma 3.2, we see that for any fixed $(u, v) \in \mathbb{B}_{2 \epsilon / 3}^{l} \times \mathbb{B}_{2 \epsilon / 3}^{l}$, $h_{u^{\prime}, v^{\prime}}^{*} P$ is $C^{0}$-isomorphic to $h_{u+u^{\prime}, v+v^{\prime}}^{*} P$ for $\mathcal{H}^{2 l}$-a.e. $\left(u^{\prime}, v^{\prime}\right) \in \mathbb{B}_{\epsilon / 3}^{l} \times \mathbb{B}_{\epsilon / 3}^{l}$. From this, as in the proof of Lemma 3.2, we see that $\left[h_{u, v}^{*} P\right]_{0}$ is independent of $\mathcal{H}^{2 l}$-a.e. $(u, v) \in \mathbb{B}_{\epsilon / 3}^{l} \times \mathbb{B}_{\epsilon / 3}^{l}$. As in Definition 3.1, we denote by $[P]_{h, K} \in \hat{\mathcal{P}}_{G}^{0}(K)$ the class $\left[h_{u, v}^{*} P\right]_{0} \in \hat{\mathcal{P}}_{G}^{0}(K)$ which is independent of $\mathcal{H}^{2 l}$ a.e. $(u, v) \in \mathbb{B}_{\epsilon / 3}^{l} \times \mathbb{B}_{\epsilon / 3}^{l}$. Once we have Lemma 5.1, arguing exactly as in the proofs of Lemma 3.3 and Lemma 3.5, we see that $[P]_{h, K}$ depends only on the homotopy class (through piecewise smooth homotopy) of $h$ and the $W^{k, p}$-isomorphism class of $P$. To prove the assertion for general Lipschitz map $h: K \rightarrow M$, we approximate $h$ by piecewise smooth $h_{n}: K \rightarrow M$ in the Lipschitz norm. Then $[P]_{h_{n}, K}$ is defined and independent of large $n$ since the homotopy class of $h_{n}$ is independent of large $n$. Similarly, the class $[P]_{h_{n}, K}$ is also independent of the approximation $\left\{h_{n}\right\}$ of $h$ since for any other approximation $\left\{h_{n}^{\prime}\right\}, h_{n}$ and $h_{n}^{\prime}$ are homotopic to each other if $n$ is large. We denote this independent class as $[P]_{h, K}$. Then its dependence on the homotopy class of $h$ and the $W^{k, p}$-isomorphism class of $P$ easily follows from the definition and the corresponding result for $h$ piecewise smooth. The last assertion of the theorem also follows as in the proof of Lemma 3.5.

Remark 5.1 When $S=\emptyset$, the class $[P]_{h, K}$ defined in Theorem 5.1 coincides with that of defined in Theorem 3.1. To see this, for $(u, v) \in \mathbb{B}_{2 \epsilon / 3}^{l} \times \mathbb{B}_{2 \epsilon / 3}^{l}$ and $v^{\prime} \in \mathbb{B}_{\epsilon / 3}^{l}$, define $\tilde{H}(t, x)=\iota_{\varphi(t) u}^{-1} \circ \pi_{M}(h(x)+\varphi(t) v)$ and $\tilde{H}_{v^{\prime}}(t, x)=$ $\iota_{\varphi(t) u}^{-1} \circ \pi_{M}\left(h(x)+\varphi(t) v+v^{\prime}\right)$, where $\varphi$ is a cut-off function as defined in the proof of Theorem 3.1. Then as in the proof of Theorem 3.1, we see that there exists $t_{0} \in[-1,0]$ and $t_{1} \in[1,2]$ such that $\left.\tilde{H}_{v^{\prime}}^{*}(P)\right|_{t_{0} \times K}$ is isomorphic to $\left.\tilde{H}_{v^{\prime}}^{*}(P)\right|_{t_{1} \times K}$ as $C^{0}$-bundles over $K$ for a.e. $v^{\prime} \in \mathbb{B}_{\epsilon / 3}^{l}$. Thus $h_{v^{\prime}}^{*} P \cong h_{u, v+v^{\prime}}^{*} P$ for a.e. $v^{\prime} \in \mathbb{B}_{\epsilon / 3}^{l}$. The assertion follows from this. 
We next extend Theorem 3.2 for bundles in $\mathcal{P}_{\text {strong,G }}^{k, p}(M ; S)$.

Theorem 5.2 Let $S \subset M$ be a closed set as in Theorem 5.1 and $P \in$ $\mathcal{P}_{\text {strong, }}^{k, p}(M ; S)$. Suppose $K$ is a d-dimensional polyhedron satisfying (A-1) and (A-2) in the appendix. Under the assumption $d \leq \min \left\{d_{\text {strong }}(k p), s-1\right\}$, where $d_{\text {strong }}(k p)$ is defined in $\S 3.2$, for any Lipschitz map $h: K \rightarrow M$, we can associate a class $[P]_{h, K}^{s} \in \hat{\mathcal{P}}_{G}^{0}(K)$ which depends only on the homotopy class of $h$ and the $W^{k, p}$-isomorphism class of $P$.

Proof. We only give a sketch of the proof. First we assume $h: K \rightarrow M$ is piecewise smooth. Since $P \in \mathcal{P}_{\text {strong, } G}^{k, p}(M ; S)$, for any compact $L \subset M \backslash S$, there exists a sequence of smooth $G$-bundles $\left\{P_{n}\right\}$ such that Definition $5.3 \mathrm{i}$ ) is satisfied. By Lemma 5.1, for a.e. $(u, v) \in \mathbb{B}_{\epsilon}^{l} \times \mathbb{B}_{\epsilon}^{l}$ we have $h_{u, v}(K) \cap S=\emptyset$. Choose any compact $L \subset M \backslash S$ such that $h_{u, v}(K) \subset L$ for $(u, v) \in \mathbb{B}_{\epsilon}^{l} \times \mathbb{B}_{\epsilon}^{l}$ of positive $\mathcal{H}^{2 l}$-measure. For such $L$, take $\left\{P_{n}\right\}$ as above. Then by the Fubinitype Lemma 3.1 and arguing as in $\S 3.2$ we see that for $\mathcal{H}^{2 l}$-a.e. $(u, v) \in$ $\mathbb{B}_{\epsilon}^{l} \times \mathbb{B}_{\epsilon}^{l}$ satisfying $h_{u, v}(K) \subset L$, the isomorphism class $\left[h_{u, v}^{*} P_{n}\right]_{0} \in \hat{\mathcal{P}}_{G}^{0}(K)$ is well-defined and independent of a.e. $(u, v) \in \mathbb{B}_{\epsilon}^{l} \times \mathbb{B}_{\epsilon}^{l}$ and the approximation $\left\{P_{n}\right\}$ satisfying Definition 5.3 i). Since $L \subset M \backslash S$ satisfying $h_{u, v}(K) \subset L$ is arbitrary, we obtain a well-defined class $[P]_{h, K}^{s} \in \hat{\mathcal{P}}_{G}^{0}(K)$ which is defined as the class $\left[h_{u, v}^{*} P_{n}\right]_{0} \in \hat{\mathcal{P}}_{G}^{0}(K)$ for a.e $(u, v) \in \mathbb{B}_{\epsilon}^{l} \times \mathbb{B}_{\epsilon}^{l}$ and for large $n$ for some approximating smooth bundles $\left\{P_{n}\right\}$ of $P$ satisfying the conditions in Definition 5.3 i). For general Lipschitz map $h: K \rightarrow M$, we approximate $h$ by piecewise smooth map $h_{n}: K \rightarrow M$. Then as in the proof of Theorem 5.1, the class $[P]_{h_{n}, K}^{s}$ does not depend on $n$ if $n$ is large and also independent of a particular choice of the approximation. We denote this common class as $[P]_{h, K}^{s}$. The remaining assertions, namely its dependence on the homotopy class of $h$ and its invariance under $W^{k, p}$-isomorphisms are proved similarly as in Lemma 3.11. This completes the proof.

As in Remark 5.1, the class $[P]_{h, K}^{s}$ defined in Theorem 5.2 coincides with that of Theorem 3.2 if $S=\emptyset$.

The next result is an extension of Thm. 3.3 for bundles in $\mathcal{P}_{\text {weak, } G}^{k, p}(M ; S)$.

Theorem 5.3 Let $S \subset M$ be a closed set as in Theorem 5.1 and $P \in$ $\mathcal{P}_{\text {weak,G }}^{k, p}(M ; S)$. Suppose $K$ is a d-dimensional polyhedron satisfying (A-1), (A-2) in the appendix. Under the assumption $d \leq \min \left\{d_{\text {weak }}(k p), s-1\right\}$, where $d_{\text {weak }}(k p)$ is defined in $\S 3.3$, for any Lipschitz map $h: K \rightarrow M$, we can associate a class $[P]_{h, K}^{w} \in \hat{\mathcal{P}}_{G}^{0}(K)$ which depends only on the homotopy class of $h$ and the $W^{k, p}$-isomorphism class of $P$.

Proof. The idea of the proof is the same as that of Theorem 5.2 and Theorem 3.3. We leave the details to the reader. 
As in the previous two results, the class $[P]_{h, K}^{w}$ defined in Theorem 5.3 coincides with that of Theorem 5.2 if $S=\emptyset$.

The following examples show that the results of Theorem 5.1, Theorem 5.2 and Theorem 5.3 are optimal.

Examples. i) Assume $P_{0} \rightarrow S^{m-1}$ is a non-trivial principal $G$-bundle of class $C^{\infty}$. Such a bundle exists if for example $G$ is a simple Lie group and $m=5$. Let $\pi: B^{m} \backslash\{0\} \rightarrow S^{m-1}$ be a radial projection, i.e., it is defined by $\pi(x)=x /|x|$. Then the bundle $P=\pi^{*} P_{0} \rightarrow B^{m}$ is a bundle of class $\mathcal{P}_{G}^{1, p}\left(B^{m} ;\{0\}\right)$ for any $1 \leq p<m$. Let $h_{0}, h_{1}: S^{m-1} \rightarrow B^{m}$ be two embeddings of the $(m-1)$-sphere in $B^{m}$ such that $h_{0}\left(S^{m-1}\right)$ does not enclose the origin 0 whereas $h_{1}\left(S^{m-1}\right)$ encloses it. Notice that $h_{0}$ is homotopic to $h_{1}$ in $B^{m}$. It is easy to see that $\left[\left(h_{0}\right)_{u, v}^{*} P\right]_{0} \in \hat{\mathcal{P}}_{G}^{0}\left(S^{m-1}\right)$ is trivial for all $(u, v) \in \mathbb{B}_{\epsilon}^{l} \times \mathbb{B}_{\epsilon}^{l}$ if $\epsilon>0$ is small. On the other hand, the class $\left[\left(h_{1}\right)_{u, v}^{*} P\right]_{0}$ coincides with $\left[P_{0}\right]$ for all $(u, v) \in \mathbb{B}_{\epsilon}^{l} \times \mathbb{B}_{\epsilon}^{l}$ if $\epsilon>0$ is small. Therefore if we take $m-1 \leq p<m$, we see that Theorem 5.1 does not hold for $\min \{s-2,[k p]-1\}=m-2$ replaced by $m-1$.

ii) In [14], we have characterized the weak Uhlenbeck closure of $C^{\infty}$ bundles in $\mathcal{P}_{G}^{1, m}(M)$. This characterization shows that $P \in \mathcal{P}_{G}^{1, m}(M)$ is a weak Uhlenbeck limit of $C^{\infty}$-bundles $P_{n} \in \mathcal{P}_{G}^{\infty}(M)$ with $P_{n} \simeq P_{0}\left(P_{0} \in\right.$ $\mathcal{P}_{G}^{\infty}(M)$ is a fixed $C^{\infty}$-bundle over $\left.M\right)$ if and only if $[P]_{m-1}=\left[P_{0}\right]_{m-1}$, where $[P]_{m-1}$ is the $(m-1)$-isomorphism class of $P$ defined in [14]. Thus in particular for any given $P_{0} \rightarrow S^{m}$, there exists a sequence of $C^{\infty}$-bundles $\left\{P_{n}\right\}$ over $S^{m}$ such that $P_{n} \simeq P_{0}$ and $\left\{P_{n}\right\}$ weakly converges (in the sense of Uhlenbeck) to the trivial bundle $S^{m} \times G \rightarrow S^{m}$. This in particular implies that there exists a finite set $S$ such that the trivial bundle belongs to $\mathcal{P}_{\text {weak }, G}^{1, p}\left(S^{m} ; S\right)$ for any $m-1<p<m$ and $G$. Considering the case where $P_{0} \rightarrow S^{m}$ is not isomorphic to the trivial bundle, we see that the conclusion of Theorem 5.3 is optimal in the sense that $\min \left\{d_{\text {weak }}(k p), s-1\right\}$ in that theorem is not replaced by $\min \left\{d_{\text {weak }}(k p)+1, s\right\}$. Also a well-known example of "bubbling off of instantons" shows that Theorem 5.2 is also optimal.

For applications, the important case is the case $s \geq[p]$ or $s \geq[p]+1$. In fact, as was mentioned in the beginning of this subsection, bundles in the class $\mathcal{P}_{G}^{1,4}(M ; S)$ where $S \subset M$ is closed with $\operatorname{dim} S \leq m-4$ appear naturally as weak limits of sequences of $C^{\infty}$-bundles over $M$ which have connections with equi-bounded Yang-Mills energies, see [21], [36] and §6. Moreover, some kinds of such connections and bundles are expected to have singular sets whose dimensions are smaller than $m-4$, see [20], [36], [30].

Bundles in the class $\mathcal{P}_{G}^{1, p}(M ; S)$ for some closed subset $S \subset M$ with $\mathcal{H}-\operatorname{dim} \leq m-[p]-1$ are constructed as follows: We assume that $M$ is triangulated. Let $Q \rightarrow M^{[p]}$ be a principal $G$-bundle of Lipschitz class. It is defined by an open covering $\left\{U_{\alpha}^{[p]}\right\}$ of $M^{[p]}$ and a gluing cocycle $\left\{g_{\alpha \beta}^{[p]}\right\}$ of Lipschitz 
class. For each $([p]+1)$-cell $e_{\alpha}$ of $M$, denote by $\pi_{\alpha}: e^{[p]+1} \backslash\left\{c_{\alpha}\right\} \rightarrow M^{[p]}$ the radial projection, where $c_{\alpha}$ is the barycenter of $e_{\alpha}$, i.e., $\pi_{\alpha}$ is a deformation retract of $e_{\alpha} \backslash\left\{c_{\alpha}\right\}$ onto $\partial e_{\alpha} \subset M^{[p]}$. From these $\pi_{\alpha}$, we obtain a Lipschitz deformation retraction $\pi_{1}: M^{[p]+1} \backslash\left\{c_{\alpha}\right\}_{\alpha \in I_{[p]+1}} \rightarrow M^{[p]}$, where $I_{[p]+1}$ is the index set of the $([p]+1)$-cells of $M$. Then the bundle $\pi_{1}^{*} Q \rightarrow M^{[p]+1} \backslash\left\{c_{\alpha}\right\}_{\alpha \in I_{[p]+1}}$ defined by an open covering $\left\{\pi_{1}^{-1}\left(U_{\alpha}^{[p]}\right)\right\}$ of $M^{[p]+1} \backslash\left\{c_{\alpha}\right\}_{\alpha \in I_{[p]+1}}$ and a gluing cocycle $\left\{\pi_{1}^{*} g_{\alpha \beta}^{[p]}\right\}$ is a bundle in the class $\mathcal{P}_{G}^{1, p}\left(M^{[p]+1} ;\left\{c_{\alpha}\right\}_{\alpha \in I_{[p]+1}}\right)$. Similarly, from deformation retractions $\pi_{2}$ : $M^{[p]+2} \backslash\left\{c_{\alpha}\right\}_{\alpha \in I_{[p]+2}} \rightarrow M^{[p]+1}, \ldots, \pi_{m-[p]}: M \backslash\left\{c_{\alpha}\right\}_{\alpha \in I_{m}} \rightarrow M^{m-1}$, we obtain a bundle $P:=\pi_{m-[p]}^{*} \cdots \pi_{1}^{*} Q \rightarrow M \backslash S$, where $S \subset M$ is defined inductively as $S_{1}=\left\{c_{\alpha}\right\}_{\alpha \in I_{[p]+1}}, S_{k}=\pi_{k}^{-1}\left(S_{k-1}\right) \cup\left\{c_{\alpha}\right\}_{\alpha \in I_{[p]+k}}$ and $S=S_{m-[p]}$.

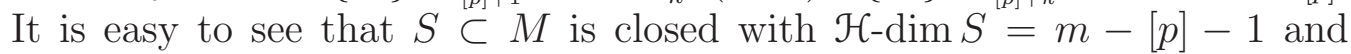
$P \in \mathcal{P}_{G}^{1, p}(M ; S)$. This bundle has the following property; its $([p]-1)$-class defined in Theorem 5.1 is $\left[\left.Q\right|_{\left.M^{[p]-1}\right]}\right] \in \hat{\mathcal{P}}_{G}^{0}\left(M^{[p]-1}\right)$. Here we consider $M$ itself as a polyhedron and the $([p]-1)$-class of $P$ is, in the notation of Theorem 5.1, $[P]_{\iota, M[p]-1}$, where $\iota: M^{[p]-1} \rightarrow M$ is the inclusion. To see this, for $u, v \in \mathbb{B}_{\epsilon}^{l}$, if $|u|$ and $|v|$ are small, we have $\iota_{u}\left(M^{[p]-1}\right) \cap S=\emptyset$ and $\iota_{v}\left(M^{[p]-1}\right) \cap S=\emptyset$ and $\iota_{u}$ and $\iota_{v}$ are homotopic to each other as maps from $M^{[p]-1}$ to $M \backslash S$. Thus we have $\iota_{u}^{*} P \simeq \iota_{v}^{*} P$ as bundles over $M^{[p]-1}$. In particular, taking $v=0$, we obtain $\left.\iota_{u}^{*} P \simeq Q\right|_{M[p]-1}$ for any small $u \in \mathbb{B}_{\epsilon}^{l}$. Thus the claim follows from the definition of the class $[P]_{\iota, M[p]-1}$.

In fact, for bundles in the class $\mathcal{P}_{G}^{1, p}(M ; S)$ for some closed $S \subset M$ with $\mathcal{H}$-dim $S \leq m-[p]-1$, the converse is also true. In the following, for simplicity of notation, we denote the class $[P]_{\iota, M^{[p]-1}}$ simply by $[P]_{M^{[p]-1}}$.

Proposition 5.1 As above, we consider $M$ itself as a polyhedron and denote by $\iota: M^{[p]-1} \rightarrow M$ the inclusion. We then have the following:

$$
\begin{aligned}
& \left\{[P]_{M^{[p]-1}}: P \in \mathcal{P}_{G}^{1, p}(M ; S) \text { for some closed } S \subset M\right. \\
& \text { with } \mathcal{H}-\operatorname{dim} S \leq m-[p]-1\}=\left\{\left[\left.Q\right|_{\left.M^{[p]-1}\right]_{0}}: Q \in \mathcal{P}_{G}^{0}\left(M^{[p]}\right)\right\} .\right.
\end{aligned}
$$

Proof. We have already shown the inclusion $\supset$. To prove the reverse inclusion, for any closed subset $S \subset M$ with $\mathcal{H}$-dim $S \leq m-[p]-1$, take any $P=\left\langle\left\{U_{\alpha}\right\},\left\{g_{\alpha \beta}\right\}\right\rangle \in \mathcal{P}_{G}^{1, p}(M ; S)$. By Lemma 5.1 and Theorem 5.1, we may assume without loss of generality that $M^{[p]} \cap S=\emptyset$. By the Fubini type theorem (see Lemma 3.1), for a.e. $u \in \mathbb{B}_{\epsilon}^{l}$, we have $\iota_{u}^{*} P=\left\langle\left\{\iota_{u}^{-1}\left(U_{\alpha}\right)\right\},\left\{g_{\alpha \beta} \circ \iota_{u}\right\}\right\rangle \in$ $\mathcal{P}_{G}^{1, p}\left(M^{[p]}\right)$, here we also denote $\iota: M^{[p]} \rightarrow M$ the inclusion. Since the bundles in the class $\mathcal{P}_{G}^{1, p}\left(M^{[p]}\right)$ have well-defined $C^{0}$-isomorphism classes, there exists $Q \in \mathcal{P}_{G}^{0}\left(M^{[p]}\right)$ such that $\left[\iota_{u}^{*} P\right]_{[p]}=[Q]_{0}$. On the other hand, by the definition of $[P]_{M[p]-1}$, we easily see that $[P]_{M^{[p]-1}}=\left[\left.\iota_{u}^{*} P\right|_{M^{[p]-1}}\right]_{0} \in \hat{\mathcal{P}}_{G}^{0}\left(M^{[p]}\right)$ for a.e. $u \in \mathbb{B}_{\epsilon}^{l}$ choosing $\epsilon>0$ small if necessary (see Theorem 5.1 and Remark 5.1). 
Recall that the isomorphism class of $Q$ as above is defined as follows. It is defined as the $C^{0}$-isomorphism class of $C^{0} \cap W^{1, p}$-bundles $Q_{\epsilon} \rightarrow M^{[p]}$ which approximate $\iota_{u}^{*} P$ in the $W^{1, p}$-sense, i.e., $Q_{\epsilon}=\left\langle\left\{V_{i}\right\},\left\{h_{i j}^{\epsilon}\right\}\right\rangle \in \mathcal{P}_{G}^{0}\left(M^{[p]}\right)$ is such that $\left\{V_{i}\right\}$ is a refinement of $\left\{\iota_{u}^{-1}\left(U_{\alpha}\right)\right\}\left(V_{i} \subset \iota_{u}^{-1}\left(U_{\varphi(i)}\right)\right)$ and

$$
\left\|g_{\varphi(i) \varphi(j)} \circ \iota_{u}-h_{i j}^{\epsilon}\right\|_{W^{1, p}\left(M^{[p]} \cap V_{i j}\right)}<\epsilon .
$$

Let $\iota_{[p]}^{[p]-1}: M^{[p]-1} \rightarrow M^{[p]}$ be the inclusion and $\iota_{[p], v}^{[p]-1}: M^{[p]-1} \rightarrow M^{[p]}$ be defined by $\iota_{[p], v}^{[p]-1}(x)=\pi_{M[p]}(x+v)$, where $\pi_{M^{[p]}}: \mathcal{O}\left(M^{[p]}\right):=\pi_{M}^{-1}\left(M_{\epsilon}^{[p]}\right) \rightarrow M^{[p]}$ is defined in $\S 7$ Appendix. As in Lemma 3.1, applying Fubini's theorem we have the following: For $f \in L^{1}\left(M^{[p]}\right)$

$$
\begin{aligned}
\int_{\mathbb{B}_{\epsilon}^{l}}\left(\int_{M^{[p]-1}} f \circ \iota_{[p], v}^{[p]-1}(x) d \mathcal{H}^{[p]-1}(x)\right) d \mathcal{H}^{l}(v) \\
\leq \int_{M^{[p]-1}}\left(\int_{\mathcal{O}\left(M^{[p]}\right)} f\left(\pi_{M^{[p]}}(v)\right) d \mathcal{H}^{l}(v)\right) d \mathcal{H}^{[p]-1}(x) \\
\leq C \int_{M^{[p]}} f(x) d \mathcal{H}^{[p]}(x) .
\end{aligned}
$$

Taking a sequence $\epsilon_{k} \downarrow 0$ and applying (5.2) to $f=\chi_{V_{i j}}\left(g_{\varphi(i) \varphi(j)} \circ \iota_{u}-h_{i j}^{\epsilon_{k}}\right)$, we see from (5.1) that for a.e $v \in \mathbb{B}_{\epsilon}^{l}$ there exists a subsequence of $\left\{\epsilon_{k}\right\}$ (still denoted by $\left.\left\{\epsilon_{k}\right\}\right)$ such that

$$
\left\|g_{\varphi(i) \varphi(j)} \circ \iota_{u} \circ \iota_{[p], v}^{[p]-1}-h_{i j}^{\epsilon_{k}} \circ \iota_{[p], v}^{[p]-1}\right\|_{W^{1, p}\left(M^{[p]-1} \cap V_{i j}\right)} \rightarrow 0
$$

as $k \rightarrow \infty$. Therefore by the Sobolev embedding theorem $W^{1, p} \subset C^{0}$ in dimension $[p]-1$, we obtain

$$
\left[\iota_{[p], v}^{[p]-1^{*}} Q\right]_{0}=\left[\iota_{[p], v}^{[p]-1^{*}} \iota_{u}^{*} P\right]_{0}
$$

for a.e $u, v \in \mathbb{B}_{\epsilon}^{l}$.

On the other hand, for $u, v, u^{\prime}, v^{\prime} \in \mathbb{B}_{\epsilon}^{l}, \iota_{u} \circ \iota_{[p], v}^{[p]-1}: M^{[p]-1} \rightarrow M$ and $\iota_{u^{\prime}} \circ$ $\iota_{[p], v^{\prime}}^{[p]-1}: M^{[p]-1} \rightarrow M$ are homotopic to each other. In fact, $H_{1}:[0,1] \times M^{[p]} \rightarrow$ $M$ defined by $H_{1}(t, x)=\pi_{M}\left(x+t u+(1-t) u^{\prime}\right)$ and $H_{2}:[0,1] \times M^{[p]-1} \rightarrow M^{[p]}$ defined by $H_{2}(t, x)=\pi_{M[p]}\left(x+t v+(1-t) v^{\prime}\right)$ give homotopies $\iota_{u} \sim \iota_{u^{\prime}}$ and $\iota_{[p], v}^{[p]-1} \sim \iota_{[p], v^{\prime}}^{[p]-1}$, respectively. Thus $H(t, x)=H_{1}\left(t, H_{2}(t, x)\right)$ gives a homotopy $\iota_{u} \circ \iota_{[p], v}^{[p]-1} \sim \iota_{u^{\prime}} \circ \iota_{[p], v^{\prime}}^{[p]-1}$. Applying the argument in the proof of Theorem 3.1 and Theorem 5.1, for a.e. $w \in \mathbb{B}_{\epsilon}^{l}$, we have $\left.\left.H_{w}^{*} P\right|_{\{0\} \times M^{[p]-1}} \simeq H_{w}^{*} P\right|_{\{1\} \times M^{[p]-1}}$ as $C^{0}$-bundles over $M^{[p]-1}$. Taking in particular $v^{\prime}=0$, we obtain

$$
\left[\iota_{[p], v}^{[p]-1^{*}} \iota_{u+w}^{*} P\right]_{0}=\left[\iota_{u^{\prime}+w}^{*} P\right]_{0}
$$

for a.e. $w \in \mathbb{B}_{\epsilon}^{l}$ as $C^{0}$-isomorphism classes of bundles over $M^{[p]-1}$. 
Similarly by the argument leading to (5.4), for a $C^{0} \cap W^{1, p}$-bundle $Q^{\prime} \rightarrow$ $M^{[p]}$ satisfying $\left[\iota_{u+w}^{*} P\right]_{[p]}=\left[Q^{\prime}\right]_{0}$ (notice that $\iota_{u+w}^{*} P \rightarrow M^{[p]}$ is a $W^{1, p}$-bundle for a.e. $w \in \mathbb{B}_{\epsilon}^{l}$ ), we have

$$
\left[\iota_{[p], v}^{[p]-1^{*}} \iota_{u+w}^{*} P\right]_{0}=\left[\iota_{[p], v}^{[p]-1^{*}} Q^{\prime}\right]_{0}
$$

for a.e. $v, w \in \mathbb{B}_{\epsilon}^{l}$ as $C^{0}$-isomorphism classes of bundles over $M^{[p]-1}$.

On the other hand, since $\iota_{[p], v}^{[p]-1} \sim \iota_{[p]}^{[p]-1}: M^{[p]-1} \rightarrow M^{[p]}$, we have $\left[\iota_{[p], v}^{[p]-1^{*}} Q^{\prime}\right]_{0}=\left[\left.Q^{\prime}\right|_{\left.M^{[p]-1}\right]_{0}}\right.$. Combining this with (5.5) and (5.6), we obtain $\left[\iota_{u^{\prime}+w}^{*} P\right]_{0}=\left[\left.Q^{\prime}\right|_{M[p]-1}\right]_{0}$ for a.e. $w \in \mathbb{B}_{\epsilon}^{l}$ as $C^{0}$-isomorphism classes of bundles over $M^{[p]-1}$. Since the class $[P]_{M^{[p]-1}}$ coincides with $\left[\iota_{u^{\prime}+w}^{*} P\right]_{0}$ for a.e. $w \in \mathbb{B}_{\epsilon}^{l}$, we finally obtain $[P]_{M[p]-1}=\left[\left.Q^{\prime}\right|_{M^{[p]-1}}\right]_{0}$. This completes the proof of the inclusion $\subset$.

\subsection{Singular Sobolev connections on singular Sobolev bundles and the Chern-Weil theory}

In this subsection, we extend results proved in $\S 4$ for singular Sobolev connections on singular Sobolev bundles.

Assume $P=\left\langle\left\{U_{\alpha}\right\}_{\alpha \in I},\left\{g_{\alpha \beta}\right\}_{\alpha, \beta \in I}\right\rangle \in \mathcal{P}_{G}^{k, p}(M ; S)$ for some closed subset $S \subset M$ with $\mathcal{H}$-dim $S=m-s$. Since $\mathcal{P}_{G}^{k, p}(M ; S) \subset \mathcal{P}_{G}^{1, k p}(M ; S)$ by the Gagliardo-Nirenberg inequality, we may assume throughout this subsection that $k=1$. Also let us assume that $A=\left\{A_{\alpha}\right\}_{\alpha \in I}$ is a connection of class $\mathfrak{A}^{1, p / 2}$ on $P$, i.e., $A_{\alpha} \in L^{p}\left(U_{\alpha}, T^{*} U_{\alpha} \otimes \mathfrak{g}\right), d A_{\alpha} \in L^{p / 2}\left(U_{\alpha}, \bigwedge^{2} T^{*} U_{\alpha} \otimes \mathfrak{g}\right)$ for any $\alpha \in I$ and (2.1) holds for $\alpha, \beta \in I$ with $U_{\alpha \beta} \neq \emptyset$. For applications in mind (see $\S 6$ ), we moreover assume that $\int_{M}\left|F_{A}\right|^{p / 2} \mathrm{dvol}_{M}<+\infty$.

For $\phi \in I^{k}(G), P_{\phi}\left(F_{A}\right) \in L^{1}\left(M, \bigwedge^{2 k} T^{*} M \otimes \mathfrak{g}\right)$ is defined as in $\S 4$. To extend the results of $\S 4$ for such classes of bundles and connections, we first need the following:

Lemma 5.2 Let $P$ and $A$ be as above. Let us assume $2 k \leq p-p / s$ and $\mathcal{H}^{m-p /(p-2 k)}(S)<+\infty$. Then we have $d P_{\phi}\left(F_{A}\right)=0$ in $\mathcal{D}^{\prime}\left(M, \bigwedge^{2 k+1} T^{*} M\right)$.

Remark 5.2 Under the assumption $2 k \leq p-p / s$, we have $m-s \leq m-$ $p /(p-2 k)$. Thus the condition $\mathcal{H}^{m-p /(p-2 k)}(S)<+\infty$ is automatically satisfied if $\mathcal{H}^{m-s}(S)<+\infty$ or $2 k<p-p / s$. Particularly interesting case is the case $s \geq[p]$. In this case $2 k \leq p-p / s$ is satisfied if $2 k \leq[p]-1$.

Proof of Lemma 5.2. Since $\mathcal{H}^{m-p /(p-2 k)}(S)=: M<+\infty$, for any small $\delta>0$, there exists open balls $\left\{B_{r_{i}}\left(x_{i}\right)\right\}_{i=1}^{N}$ with $r_{i}<\delta, x_{i} \in S$ such that $S \subset \bigcup_{i=1}^{N} B_{r_{i}}\left(x_{i}\right)$ and

$$
\sum_{i=1}^{N} r_{i}^{m-p /(p-2 k)} \leq M+1
$$


Let $\phi_{i} \in C^{\infty}(M)$ be a function such that $\phi_{i}=0$ in $B_{r_{i}}\left(x_{i}\right), \phi_{i}=1$ in $M \backslash B_{2 r_{i}}\left(x_{i}\right), 0 \leq \phi_{i} \leq 1$ in $M$ and $\left|\nabla \phi_{i}\right| \leq C r_{i}^{-1}$ for some constant $C>0$ depending only on $M$. Set $\phi_{\delta}(x)=\min _{1 \leq i \leq N} \phi_{i}(x)$. Then $\phi_{\delta}$ satisfies $\operatorname{supp}\left(\phi_{\delta}\right) \subset M \backslash S, \phi_{\delta}=1$ in $M \backslash N_{2 \delta}(S)$, where $N_{2 \delta}(S)=\{x \in M$ : $d(x, S) \leq 2 \delta\}$.

For any $\alpha \in C^{\infty}\left(M, \bigwedge^{m-2 k-1} T^{*} M\right)$, we have

$$
\int_{M} P_{\phi}\left(F_{A}\right) \wedge d \alpha=\int_{M} P_{\phi}\left(F_{A}\right) \wedge d\left(\phi_{\delta} \alpha\right)+\int_{M} P_{\phi}\left(F_{A}\right) \wedge d\left(\left(1-\phi_{\delta}\right) \alpha\right)
$$

The first term on the right side of (5.8) is 0 by the same reasoning as in the proof of Lemma 4.1. The second term is estimated as

$$
\begin{aligned}
& \mid \int_{M} P_{\phi}\left(F_{A}\right) \wedge d\left(\left(1-\phi_{\delta}\right) \alpha\right) \mid \\
& \leq C \int_{M}\left|F_{A}\right|^{k}\left|\nabla \phi_{\delta}\right||\alpha| \mathrm{dvol}_{M}+C \int_{N_{2 \delta}(S)}\left|F_{A}\right|^{k}|d \alpha| \mathrm{dvol}_{M} .
\end{aligned}
$$

Since $2 k \leq p$ and $\int_{M}\left|F_{A}\right|^{p / 2} \mathrm{dvol}_{M}<+\infty$, the second term on the right of (5.9) tends to 0 as $\delta \rightarrow 0$. As for the first term, we have by the Hölder's inequality and (5.7)

$$
\begin{aligned}
& \int_{N_{2 \delta}(S)}\left|F_{A}\right|^{k}\left|\nabla \phi_{\delta}\right||\alpha| \mathrm{dvol}_{M} \\
& \leq\|\alpha\|_{L^{\infty}(M)}\left(\int_{N_{2 \delta}(S)}\left|F_{A}\right|^{p / 2} \mathrm{dvol}_{M}\right)^{2 k / p}\left(\int_{N_{2 \delta}(S)}\left|\nabla \phi_{\delta}\right|^{p /(p-2 k)} \mathrm{dvol}_{M}\right)^{1-2 k / p} \\
& \leq\|\alpha\|_{L^{\infty}(M)}\left(\int_{N_{2 \delta}(S)}\left|F_{A}\right|^{p / 2} \operatorname{dvol}_{M}\right)^{2 k / p}\left(\sum_{i=1}^{N} \int_{N_{2 \delta}(S)}\left|\nabla \phi_{i}\right|^{p /(p-2 k)} \mathrm{dvol}_{M}\right)^{1-2 k / p} \\
& \leq\|\alpha\|_{L^{\infty}(M)}\left(\int_{N_{2 \delta}(S)}\left|F_{A}\right|^{p / 2} \operatorname{dvol}_{M}\right)^{2 k / p}\left(\sum_{i=1}^{N} \int_{B_{2 r_{i}}\left(x_{i}\right)}\left|\nabla \phi_{i}\right|^{p /(p-2 k)} \mathrm{dvol}_{M}\right)^{1-\frac{2}{p} k} \\
& \leq C\|\alpha\|_{L^{\infty}(M)}\left(\int_{N_{2 \delta}(S)}\left|F_{A}\right|^{p / 2} \operatorname{dvol}_{M}\right)^{2 k / p}\left(\sum_{i=1}^{N} r_{i}^{m-p /(p-2 k)}\right)^{1-2 k / p}
\end{aligned}
$$

$$
\leq C(M+1)^{1-2 k / p}\|\alpha\|_{L^{\infty}(M)}\left(\int_{N_{2 \delta}(S)}\left|F_{A}\right|^{p / 2} \operatorname{dvol}_{M}\right)^{2 k / p}
$$

and (5.10) tends to 0 as $\delta \rightarrow 0$.

Therefore we have $\int_{M} P_{\phi}\left(F_{A}\right) \wedge d \alpha=0$ for any $\alpha \in C^{\infty}\left(M, \bigwedge^{m-k-1} T^{*} M\right)$. This completes the proof. 
Once Lemma 5.2 is proved, analogues of Lemmas 4.2, 4.3, 4.4 and 4.5 hold. Thus $P_{\phi}(A)$ defines a cohomology class in $H^{2 k}(M ; \mathbb{R})$ under the assumption of $A$ at the beginning of this section. On the other hand, as in $\S 4$, we can also define a current $C_{\phi}(A)$ by the formula (4.24) for $A$ as above and $\phi \in I^{k}(G)$. Then by Lemma 5.2, as in $\S 4, C_{\phi}(A)$ defines a homology class in $H_{m-2 k}(M ; \mathbb{R})$. Arguing exactly as in the proof of Proposition 4.1, we see that $\left[P_{\phi}(A)\right]$ is Poincaré dual to $\left[C_{\phi}(A)\right]$, i.e., the conclusion of Proposition 4.1 also holds in this case. To complete the Chern-Weil theory in this setting, it only remains to show that the cohomology class $\left[P_{\phi}(A)\right] \in H^{2 k}(M ; \mathbb{R})$ is independent of $A \in \mathfrak{A}^{1, p / 2}(P)$. Here we can not argue as in the proof of Theorem 4.1 since under our assumption, the curvature of the connection $A=\left\{A_{\alpha}(x, t)\right\}$ in the proof of Theorem 4.1 does not belong to $L^{p / 2}(M)$ in general. To overcome this, we argue as follows.

Let $P=\left\langle\left\{U_{\alpha}\right\},\left\{g_{\alpha \beta}\right\}\right\rangle \in \mathcal{P}_{G}^{1, p}(M ; S)$ and $A_{1}, A_{2} \in \mathfrak{A}^{1, p / 2}(P)$, where $S \subset M$ is a closed subset with $\sigma$-finite $\mathcal{H}^{m-s}$-measure. To prove $\left[P_{\phi}\left(A_{1}\right)\right]=$ $\left[P_{\phi}\left(A_{2}\right)\right]$, it suffices to show that for any $2 k$-dimensional submanifold $\Sigma \subset M$, we have $\left\langle\left[P_{\phi}\left(A_{1}\right)\right],[\Sigma]\right\rangle=\left\langle\left[P_{\phi}\left(A_{2}\right)\right]\right.$, [ $\left.[\Sigma]\right\rangle$, i.e., for a.e. $(u, v) \in \mathbb{B}_{\epsilon}^{l} \times \mathbb{B}_{\epsilon}^{l}$, we have

$$
\int_{\Sigma_{u, v}} P_{\phi}\left(A_{1}\right)=\int_{\Sigma_{u, v}} P_{\phi}\left(A_{2}\right)
$$

where $\Sigma_{u, v}=\iota_{u}^{-1} \circ \iota_{v}(\Sigma)$.

To see (5.11), recall from Lemma 5.1 that for a.e. $(u, v) \in \mathbb{B}_{\epsilon}^{l} \times \mathbb{B}_{\epsilon}^{l}$, we have $\Sigma_{u, v} \cap S=\emptyset$ provided $2 k<s$. Moreover, by the Fubini type lemma, for a.e. $(u, v) \in \mathbb{B}_{\epsilon}^{l} \times \mathbb{B}_{\epsilon}^{l}$ we see that $\left.P\right|_{\Sigma_{u, v}} \rightarrow \Sigma_{u, v}$ is a bundle of class $W^{1, p} \subset C^{0}$ (since $2 k<p$ under the assumption of Lemma 5.2) and $\left.A_{1}\right|_{\Sigma_{u, v}},\left.A_{2}\right|_{\Sigma_{u, v}} \in \mathfrak{A}^{1, p / 2}\left(\left.P\right|_{\Sigma_{u, v}}\right)$. Since such bundle $\left.P\right|_{\Sigma_{u, v}}$ and connections $\left.A_{1}\right|_{\Sigma_{u, v}},\left.A_{2}\right|_{\Sigma_{u, v}}$ are approximated in $W^{1, p}$ and $\mathfrak{A}^{1, p / 2}$ respectively by smooth ones (see [13]), we see that $\int_{\Sigma_{u, v}} P_{\phi}\left(A_{i}\right)(i=1,2)$ is a characteristic number of the bundle $\left.P\right|_{\Sigma_{u, v}} \rightarrow \Sigma_{u, v}$. In particular it does not depend on a particular choice of a connection and (5.11) holds.

Summing up the above argument, we obtain

Theorem 5.4 Let $P$ and $A$ be as in the beginning of this section. Assume $2 k \leq p-p / s, 2 k<s, S \subset M$ is closed with $\sigma$-finite $\mathcal{H}^{m-s}$-measure and $\mathcal{H}^{m-p /(p-2 k)}(S)<+\infty$. Then for $\phi \in I^{k}(G)$, the cohomology class $\left[P_{\phi}(A)\right] \in$ $H^{2 k}(M ; \mathbb{R})$ and the homology class $\left[C_{\phi}(A)\right] \in H_{m-2 k}(M ; \mathbb{R})$ are independent of $A \in \mathfrak{A}^{1, p / 2}(P)$. Moreover, we have $P D \circ \Psi^{-1}\left(\left[P_{\phi}(A)\right]\right)=C_{\phi}(A)$.

Stability result such as Proposition 4.2 also holds in the setting of this section. Details are left to the reader. 
We end this subsection by giving a simple example which shows that the result of Theorem 5.4 is optimal in general.

Example. Suppose $m>1$ and consider a smooth principal $G$-bundle $P_{0} \rightarrow$ $S^{m-1}$. Denote by $S^{m-1} \subset S^{m}$ the equatorial $(m-1)$-sphere. Let $N$ and $S$ be north and south poles of $S^{m}$ respectively and $r: S^{m} \backslash\{N, S\} \rightarrow S^{m-1}$ the radial retraction. We consider the pull back bundle $r^{*} P_{0} \rightarrow S^{m} \backslash\{N, S\}$. Considered as a bundle over $S^{m}$, it defines a bundle in the class $\mathcal{P}_{G}^{1, p}\left(S^{m} ;\{S, N\}\right)$ for any $1 \leq p<m$. Suppose $A_{0} \in \mathcal{A}^{\infty}\left(P_{0}\right)$ is a smooth connection on $P_{0}$. Then $A:=r^{*} A_{0}$ defines a $\mathfrak{A}^{1, p / 2}$-connection on $r^{*} P_{0}$ (considered as a bundle in the class $\left.\mathcal{P}_{G}^{1, p}\left(S^{m} ;\{N, S\}\right)\right) ; A \in \mathfrak{A}^{1, p / 2}\left(r^{*} P_{0}\right)$. We take in particular $p=m-1$. We claim that for $\phi \in I^{k}(G)$ with $2 k=m-1$ (thus it is necessary that $m$ is odd), $P_{\phi}(A)$ does not define a well-defined class in $H^{2 k}\left(S^{m}, \mathbb{R}\right)$. To see this, we first observe that $* d P_{\phi}(A)= \pm C_{0}\left(\delta_{N}-\delta_{S}\right)$, where $C_{0}=\int_{S^{m-1}} P_{\phi}\left(A_{0}\right)$ and $\delta_{N}$ and $\delta_{S}$ denote Dirac measures with unit mass at $N$ and $S$, respectively. (The sign \pm depends on the choice of the orientation of $\left.S^{m-1} \subset S^{m}\right)$. Thus if an embedded $(m-1)$-sphere $\Sigma \subset S^{m}$ does not intersect $N$ and $S$ and contractible on $S^{m} \backslash\{N, S\}$, we have $\int_{\Sigma} P_{\phi}(A)=0$. Since $\Sigma$ is homologous to $S^{m-1}$ in $S^{m}, P_{\phi}(A)$ does not define a well-defined cohomology class if $C_{0} \neq 0$. This example shows that the assumption $2 k \leq p-p / s$ in Theorem 5.4 is not relaxed in general.

\subsection{A compactness of weakly Neather Yang-Mills connections}

To illustrate an application of the results proved in $\S 5.1$, we prove in this subsection a compactness property of some kind of connections and bundles.

We first consider a smooth $G$-bundle $P \rightarrow M$ and a smooth connection $A_{0}$ on it. Assume $\left\{\phi_{t}\right\}_{|t|<\epsilon}$ is a smooth one parameter family of diffeomorphisms on $M$ such that $\phi_{0}=\mathrm{id}_{M}$. Consider the pull back connection $A^{t}:=\phi_{t}^{*}(A)$ on the bundle $\phi_{t}^{*} P \rightarrow M$. We then have the following first variational formula (see [23], [36]):

$\left.\frac{d}{d t}\right|_{t=0} Y M\left(A^{t}\right)=-\int_{M}\left(\left|F_{A_{0}}\right|^{2} \operatorname{div} X-4 \sum_{i, j=1}^{m}\left\langle F_{A_{0}}\left(\nabla_{e_{i}} X, e_{j}\right), F_{A_{0}}\left(e_{i}, e_{j}\right)\right\rangle\right) \operatorname{dvol}_{M}$,

where $X$ is the vector field generated by $\left\{\phi_{t}\right\}$, i.e., $X=\left.\frac{\partial \phi_{t}}{\partial t}\right|_{t=0}$ and $e_{i}$ $(1 \leq i \leq m)$ is an (local) orthonormal frame of $T M$.

We will call a connection $A_{0}$ Neather Yang-Mills connection if the right hand side of (5.12) equals to 0 for any vector field $X$. Thus $A_{0}$ is a Neather Yang-Mills if

$$
\int_{M}\left(\left|F_{A_{0}}\right|^{2} \operatorname{div} X-4 \sum_{i, j=1}^{m}\left\langle F_{A_{0}}\left(\nabla_{e_{i}} X, e_{j}\right), F_{A_{0}}\left(e_{i}, e_{j}\right)\right\rangle\right) \operatorname{dvol}_{M}=0
$$

holds for any vector filed $X$. 
For example if $A_{0}$ is a smooth Yang-Mills connection, it is also a Neather Yang-Mills. More generally, let $P=\left\langle\left\{U_{\alpha}\right\},\left\{g_{\alpha \beta}\right\}\right\rangle \in \mathcal{P}_{G}^{2,2}(M)$ and $A=$ $\left\{A_{\alpha}\right\} \in \mathcal{A}^{1,2}(P)$ be a Sobolev bundle and a Sobolev connection, respectively. We say that $A$ is a weakly Neather Yang-Mills connection if $A$ satisfies (5.13) for any smooth vector field $X$. For such a weakly Neather Yang-Mills connection $A$, we have the following monotonicity inequality ([23], [36]): For $a \in M$, there exists $C>0$ such that for $0<\sigma<\rho<\rho_{a}$ ( $\rho_{a}$ is the injectivity radius of $M$ at $a$ )

$$
\rho^{4-m} e^{C \rho^{2}} \int_{B_{\rho}(a)}\left|F_{A}\right|^{2} \operatorname{dvol}_{M}-\sigma^{4-m} e^{C \sigma^{2}} \int_{B_{\sigma}(a)}\left|F_{A}\right|^{2} \mathrm{dvol}_{M}
$$

$$
\geq 4 \int_{B_{\rho}(a) \backslash B_{\sigma}(a)} r^{4-m} e^{C r^{2}}\left|\iota\left(\frac{\partial}{\partial r}\right) F_{A}\right|^{2} \operatorname{dvol}_{M} .
$$

In particular, (5.14) implies that $r^{4-m} e^{C r^{2}} \int_{B_{r}(a)}\left|F_{A}\right|^{2} \mathrm{dvol}_{M}$ is monotonically non-decreasing with respect to $r$.

Let us consider $P \in \mathcal{P}_{G}^{\infty}(M)$ and a sequence $\left\{A_{n}\right\} \subset \mathcal{A}^{1,2}(P)$ of weakly Neather Yang-Mills connections satisfying a uniform energy bound:

$$
\sup _{n \geq 1} \int_{M}\left|F_{A_{n}}\right|^{2} \operatorname{dvol}_{M}<\infty \text {. }
$$

Moreover, we assume that each $A_{n}$ is approximable in the sense that (see [20]): For any $\epsilon>0$, there exists $\alpha>0$ such that for all $a \in M$ and $\rho_{M}>r_{0}>0\left(\rho_{M}\right.$ is the injectivity radius of $\left.M\right)$ if

$$
\sup _{x \in B_{r_{0}}(a), 0<r<r_{0}} r^{4-m} \int_{B_{r}(x)}\left|F_{A_{n}}\right|^{2} \operatorname{dvol}_{M}<\alpha,
$$

there there exists $\left\{A_{i}\right\} \subset C^{\infty}\left(B_{\rho_{M}}(a), T^{*} B_{\rho_{M}}(a) \otimes \mathfrak{g}\right)$ such that $A_{i} \rightarrow A_{n}$ in $W^{1,2}\left(B_{\rho_{M}}(a)\right) \cap L^{4}\left(B_{\rho_{M}}(a)\right)$ and

$$
\sup _{x \in B_{r_{0} / 2}(a), 0<r<r_{0} / 2} r^{4-m} \int_{B_{r}(x)}\left|F_{A_{i}}\right|^{2} \operatorname{dvol}_{M}<\epsilon \quad \text { for all } i .
$$

Notice that smooth connections are obviously approximable in the above sense. We then have the following:

Proposition 5.2 Let $\left\{A_{n}\right\}$ be as above. Then there exist a subsequence of $\left\{A_{n}\right\}$ (still denote by $\left\{A_{n}\right\}$ ), a closed set $S \subset M$ such that $\mathcal{H}^{m-4}(S)<+\infty$, $P_{\infty} \in \mathcal{P}_{\text {weak, } G}^{2,2}(M ; S)$ and $A_{\infty} \in \mathcal{A}^{1,2}\left(P_{\infty}\right)$ such that for a suitable gauge $\left\{A_{n}\right\}$ weakly converges to $A_{\infty}$ in $W_{\text {loc }}^{1,2}(M \backslash S)$. Moreover, for any 3-dimensional polyhedron $K$ and a Lipschitz map $h: K \rightarrow M$, we have $[P]_{[h], K}^{w}=\left[P_{\infty}\right]_{[h], K}^{w}$. 
Before beginning the proof, we need to give some comments about the statement of the proposition. Firstly, the weak convergence in the above proposition means that for any compact $K \subset M \backslash S$, there exists a subsequence of $\left\{A_{n}\right\}$ (still denoted by $\left\{A_{n}\right\}$ ), a family of open sets $\left\{U_{\alpha}\right\}$ of $M \backslash S$ such that $K \subset \bigcup_{\alpha} U_{\alpha}$ and $P$ and $P_{\infty}$ are trivialized over each $U_{\alpha}$, i.e., we can write $\left.P\right|_{K}=\left\langle\left\{U_{\alpha} \cap K\right\},\left\{g_{\alpha \beta}\right\}\right\rangle$ and $A_{n}=\left\{A_{\alpha ; n}\right\}$ with $A_{\alpha ; n} \in$ $W^{1,2}\left(U_{\alpha}, T^{*} U_{\alpha} \otimes \mathfrak{g}\right)$ and $\left.P_{\infty}\right|_{K}=\left\langle\left\{U_{\alpha} \cap K\right\},\left\{g_{\alpha \beta}\right\}\right\rangle$ and $A_{\infty}=\left\{A_{\alpha ; \infty}\right\}$ with $A_{\alpha ; \infty} \in W^{1,2}\left(U_{\alpha}, T^{*} U_{\alpha} \otimes \mathfrak{g}\right)$ such that $A_{\alpha ; n} \rightarrow A_{\alpha ; \infty}$ weakly in $W^{1,2}\left(U_{\alpha} \cap K\right)$. Under such a condition, it is easily seen that $P_{\infty} \in \mathcal{P}_{\text {weak, } G}^{2,2}(M ; S)$ and the 3-class $\left[P_{\infty}\right]_{[h], K}^{w}$ is defined by Theorem 5.3. We also remark that it can be shown that the above result extends to the case where $P \in \mathcal{P}_{G}^{2,2}(M)$. For this, we need to slightly extend the class of bundles $\mathcal{P}_{\text {weak, } G}^{2,2}(M ; S)$ in order to include the weak limit $P_{\infty}$ and show that for such bundles the 3-class is well-defined. These are done by the similar argument given in $\S 5.1$. But for simplicity we only consider the case $P \in \mathcal{P}_{G}^{\infty}(M)$.

Proof of Proposition 5.2. Define

$$
S:=\bigcap_{0<r<\rho_{M}}\left\{x \in M: \liminf _{n \rightarrow \infty} e^{a r^{2}} r^{4-m} \int_{B_{r}(x)}\left|F_{A_{n}}\right|^{2} \mathrm{dvol}_{M} \geq 2^{4-m} \alpha\right\},
$$

where $\alpha>0$ is a constant $\kappa(m)$ of Theorem 1.3 in [20].

By the monotonicity inequality (5.14) it is easily seen that $S \subset M$ is closed and a simple covering argument shows that $\mathcal{H}^{m-4}(S)<\infty$ (see [36]).

Assume $x_{0} \notin S$. Then there exists $r_{0}>0$ such that

$$
\liminf _{n \rightarrow \infty} e^{a r_{0}^{2}} r_{0}^{4-m} \int_{B_{r_{0}}\left(x_{0}\right)}\left|F_{A_{n}}\right|^{2} \operatorname{dvol}_{M}<2^{4-m} \alpha .
$$

Thus by the monotonicity inequality, we have for any $x \in B_{r_{0} / 2}\left(x_{0}\right)$ and $0<r<r_{0} / 2$

$$
\begin{aligned}
e^{a r^{2}} r^{4-m} \int_{B_{r}(x)}\left|F_{A_{n}}\right|^{2} \mathrm{dvol}_{M} & \leq e^{a r_{0}^{2} / 4}\left(\frac{r_{0}}{2}\right)^{4-m} \int_{B_{r_{0} / 2}(x)}\left|F_{A_{n}}\right|^{2} \mathrm{dvol}_{M} \\
& \leq 2^{m-4} e^{a r_{0}^{2}} r_{0}^{4-m} \int_{B_{r_{0}}\left(x_{0}\right)}\left|F_{A_{n}}\right|^{2} \operatorname{dvol}_{M}<\alpha .
\end{aligned}
$$

Since each $A_{n}$ is assumed to be approximable, we can apply the Coulomb gauge theorem of Meyer-Rivière [20, Theorem 1.3]. Thus there exists $g_{n} \in$ $W^{2,2}\left(B_{r_{0} / 2}\left(x_{0}\right), G\right) \cap W^{1,4}\left(B_{r_{0} / 2}\left(x_{0}\right), G\right)$ such that $\tilde{A}_{n}:=g_{n}^{*} A_{n}$ is in the Coulomb gauge, i.e.,

$$
\begin{array}{ll}
d^{*} \tilde{A}_{n}=0 & \text { in } B_{r_{0} / 2}\left(x_{0}\right), \\
\iota_{\nu} \tilde{A}_{n}=0 & \text { on } \partial B_{r_{0} / 2}\left(x_{0}\right),
\end{array}
$$


where $\nu$ is the outer normal of $\partial B_{r_{0} / 2}\left(x_{0}\right)$, and satisfies

$$
\left\|\tilde{A}_{n}\right\|_{W^{1,2}\left(B_{r_{0} / 2}\left(x_{0}\right)\right)}+\left\|\tilde{A}_{n}\right\|_{L^{4}\left(B_{r_{0} / 2}\left(x_{0}\right)\right)} \leq C(m)\left\|F_{A_{n}}\right\|_{L^{2}(M)} .
$$

Suppose $K \Subset M \backslash S$ is a compact subset. There exists $\left\{B_{r_{i}}\left(x_{i}\right)\right\}_{i=1}^{p}$ such that $K \subset \bigcup_{i=1}^{p} B_{r_{i}}\left(x_{i}\right), B_{r_{i}}\left(x_{i}\right) \subset M \backslash S$ for any $1 \leq i \leq p$ and on each $B_{r_{i}}\left(x_{i}\right)$ there exists $g_{i ; n} \in W^{2,2}\left(B_{r_{i}}\left(x_{i}\right), G\right)$ such that $\tilde{A}_{i ; n}:=g_{i ; n}^{*} A_{n}$ satisfies (5.19) and (5.20) for $B_{r_{0} / 2}\left(x_{0}\right)$ replaced by $B_{r_{i}}\left(x_{i}\right)$. We may assume $B_{r_{i}}\left(x_{i}\right) \subset U_{\alpha_{i}}$ for some index $\alpha_{i}$. Defining $\tilde{g}_{i j ; n}:=g_{i ; n}^{-1} g_{\alpha_{i} \alpha_{j}} g_{j ; n}$, we see that $\hat{A}_{i ; n}:=g_{i ; n}^{*} A_{\alpha_{i} ; n}$ satisfies

$$
\begin{aligned}
\hat{A}_{j ; n} & =g_{j ; n}^{*} A_{\alpha_{j} ; n}=g_{j ; n}^{*}\left(g_{\alpha_{i} \alpha_{j}}^{*} A_{\alpha_{i} ; n}\right) \\
& =g_{j ; n}^{*} g_{\alpha_{i} \alpha_{j}}^{*}\left(g_{i ; n}^{-1}\right)^{*} \hat{A}_{i ; n} \\
& =\left(g_{i ; n}^{-1} g_{\alpha_{i} \alpha_{j}} g_{j ; n}\right)^{*} \hat{A}_{i ; n} .
\end{aligned}
$$

That is, $g_{n}:=\left\{g_{i ; n}\right\}$ defines a $W^{2,2} \cap W^{1,4}$-gauge transformation on $K$ and $\left\{g_{n}^{*} A_{n}\right\}$ is $W^{1,2} \cap L^{4}$-bounded over $K$. Thus there exists a subsequence of $\left\{A_{n}\right\}$ (still denote by $\left\{A_{n}\right\}$ ) such that $\left\{g_{n}^{*} A_{n}\right\}$ weakly converges in $W^{1,2} \cap$ $L^{4}(K)$. Then the gluing cocyle $\left\{g_{i j ; n}\right\}$ also weakly converges in $W^{2,2} \cap$ $W^{1,4}(K)$. Since $K \Subset M \backslash S$ is arbitrary, a diagonal sequence argument shows that there exists a subsequence of $\left\{P_{n}\right\}$ (still denoted by $\left\{P_{n}\right\}$ ) such that $\left\{P_{n}\right\}$ weakly converges to some bundle $P_{\infty}$ in $\mathcal{P}_{\text {weak, } G}^{k, p}(M ; S)$. For this bundle, we have $[P]_{[h], K}^{w}=[P]_{[h], K}^{w}$. This completes the proof.

\section{Some variational problems}

In this section, we consider a variational problem

$$
m_{p}(P ; G):=\inf \left\{\int_{M}\left|F_{A}\right|^{p} \operatorname{dvol}_{M}: A \in \mathcal{A}^{\infty}(P)\right\}
$$

for a smooth principal $G$-bundle $P \rightarrow M$ and $1 \leq p \leq \infty$. Here the norm of the curvature $F_{A} \in C^{\infty}\left(M, \bigwedge^{2} T^{*} M \otimes \operatorname{Aut}(P)\right)$ is taken with respect to the Riemannian metric on $M$ and the bi-invariant metric on $G$. Since the norm $\left|F_{A}\right|$ is gauge invariant, $m_{p}(P ; G)$ depends only on the isomorphism class of $P$. It is also known from the work of Uhlenbeck [38] that $m_{p}(P ; G)$ is attained for any $P \rightarrow M$ provided $p>m / 2$. It is not true, however, in general that $m_{p}(P ; G)$ is attained for the case $p \leq m / 2$. For example, if $p<m / 2$, using the conformal dilation of $S^{m}$ it is easy to see that $m_{p}(P ; G)=0$ for any $P \rightarrow S^{m}$. Thus $m_{p}(P ; G)$ is not attained if $P \rightarrow S^{m}$ is not trivial. For the case $p=m / 2$, some partial results are known. A particularly interesting case is the case $m=4$ and $p=2$. In this case $m_{2}(P ; G)$ 
is attained by a special kind of solutions to the Yang-Mills equations, the (anti-)self-dual connections. This particularly interesting case is studied in [31], [32], [6] and [7]. The direct variational approach in this case is given in [27] based on the Uhlenbeck's result [38]. The case $p=m / 2$ with $m \geq 3$ is recently studied in [13] via the direct variational methods.

As we have observed above, $m_{p}(P ; G)$ is not attained in general and the general existence result is unknown for the case $p<m / 2$. To understand the problem (6.1) in this case, we need to understand the quantity $m_{p}(P ; G)$ more deeply. The above observed example indicates that $m_{p}(P ; G)$ is determined by coarser invariants of $P \rightarrow M$ than the ordinary isomorphism class of $P$. (This is not the case for $p \leq m / 2$. For example, recall that $m_{2}(M ; G)$ determines completely the isomorphism class of $P \rightarrow M$ for $m=4$ and $G$ a simple Lie group). In this section, we first introduce an invariant of $P \rightarrow M$ which is coarser than the ordinary isomorphism class of $P$, but it completely determines the value $m_{p}(P ; G)$. The introduction of the following invariant is inspired by the corresponding invariant for mappings between manifolds introduced by White [41].

Definition 6.1 Assume $M$ is triangulated. Let $P \rightarrow M$ be a smooth principal $G$-bundle. For $1 \leq k \leq m$, the $k$-class of $P$ is defined as the isomorphism class of the restriction $\left.P\right|_{M^{k}} \rightarrow M^{k}$, where $M^{k}$ is the $k$-skeleton of the triangulation. We denote the $k$-class of $P$ by $[P]_{k}$.

Notice that the $k$-class does not depend on the particular choice of the triangulation in the following sense: Suppose $P \rightarrow M$ and $Q \rightarrow M$ are principal $G$-bundles. If $M^{\prime k}$ is the $k$-skeleton of another triangulation of $M$ and $\left.P\right|_{M^{k}} \rightarrow M^{k}$ is isomorphic to $\left.Q\right|_{M^{k}} \rightarrow M^{k}$, then $\left.P\right|_{M^{\prime k}} \rightarrow M^{\prime k}$ is also isomorphic to $\left.Q\right|_{M^{\prime k}} \rightarrow M^{\prime k}$. To see this, by the cellular approximation theorem there exists a continuous map $\varphi: M \rightarrow M$ which is cellular with respect to these triangulations, i.e., $\varphi\left(M^{\prime k}\right) \subset M^{k}$ and homotopic to the identity. By the assumption, we have $\left.\left.\varphi^{*} P\right|_{M^{\prime k}} \simeq \varphi^{*} Q\right|_{M^{\prime k}}$. Since $\varphi$ is homotopic to the identity, we also have $\varphi^{*} P \simeq P$ and $\varphi^{*} Q \simeq Q$. Therefore we obtain $\left.\left.P\right|_{M^{\prime k}} \simeq Q\right|_{M^{\prime k}}$ as asserted.

The following is the first main result of this section.

Theorem 6.1 Let $P \rightarrow M$ and $Q \rightarrow M$ be smooth principal $G$-bundles with the same $[2 p]$-class, $[P]_{[2 p]}=[Q]_{[2 p]}$. Then we have $m_{p}(P ; G)=m_{p}(Q ; G)$.

The above is the analogue of the result of White [41, Corollary 1] for the mapping problem. In our case, however, the converse of the above theorem does not hold in general. For example, suppose $P \rightarrow M$ is the trivial bundle and $Q \rightarrow M$ is a flat bundle, then we have $m_{p}(P ; G)=m_{p}(Q ; G)=0$ for any $p \geq 1$ but $\left.Q\right|_{M^{[2 p]}} \rightarrow M^{[2 p]}$ is not necessary trivial in general. 
To prove Theorem 6.1, we need some preparations. Following [41] and [10], we first introduce a function $|\cdot|_{k}: M \rightarrow \mathbb{R}_{\geq 0}$ for $1 \leq k \leq m-1$. Let $K$ be an $m$-dimensional polyhedron and $h: K \rightarrow M$ a bi-Lipschitz map which gives the triangulation of $M$. For each cell $e \subset K$, we denote by $c_{e} \in \stackrel{\circ}{e}$ the barycenter of $e$. For $x \in K^{k}$, we define $|x|_{k}=1$. Assume inductively that $|x|_{k}$ is defined for $x \in K^{i-1}(k+1 \leq i \leq m)$. We then define for $i$-cell $e \subset K$ and $x \in e,|x|_{k}=\left.\left.|x|_{e}\left|x_{e}+\left(x-x_{e}\right) /\right| x\right|_{e}\right|_{k}$, where $|x|_{e}:=\inf \left\{t>0: x \in x_{e}+t\left(e-x_{e}\right)\right\}$. Notice that for $x \in e$, we have $x_{e}+\left(x-x_{e}\right) /|x|_{e} \in \partial e$ and $\left.\left.\left|x_{e}+\left(x-x_{e}\right) /\right| x\right|_{e}\right|_{k}$ is defined by our inductive hypothesis. Notice also that if $x \in \partial e$, we then have $|x|_{e}=1$ and $|x|_{k}$ defined above coincides with the one already defined on $K^{i-1}$. From these observations, we inductively define a function $|\cdot|_{k}: K \rightarrow \mathbb{R}_{\geq 0}$. Composing with $h^{-1}$, we obtain a function on $M$ which is also denoted by $|\cdot|_{k}: M \stackrel{h^{-1}}{\longrightarrow} K \stackrel{|\cdot|_{k}}{\longrightarrow} \mathbb{R}_{\geq 0}$.

For $1 \leq k \leq m-1$ and $0 \leq \epsilon \leq 1$, define $\Gamma_{\epsilon}^{k}:=\left\{x \in K:|x|_{k}=\epsilon\right\}$. Notice that $\Gamma_{1}^{k}=K^{k}$ and $\Gamma_{0}^{k}$ is the dual $(m-k-1)$-skeleton of $K$. For $0<\epsilon \leq 1$, we define $\Phi_{\epsilon}^{k}:\left\{x \in K: 0<|x|_{k} \leq 1\right\} \rightarrow \Gamma_{\epsilon}^{k}$ as follows: First $\Phi_{1}^{k}$ is defined as $\Phi_{1}^{k}(x)=x$ for $x \in K^{k}$ and for $(k+1)$-cell $e \subset K$ and $x \in e$, $\Phi_{1}^{k}(x)=x_{e}+(x-y) /|x|_{e}$. This defines $\Phi_{1}^{k}$ on $\left\{x \in K^{k+1}: 0<|x|_{k} \leq 1\right\}$. Suppose inductively that $\Phi_{1}^{k}$ is defined on $\left\{x \in K^{i-1}: 0<|x|_{k} \leq 1\right\}$ for some $k+2 \leq i \leq m$. Then for $x \in K^{i} \backslash K^{i-1}$ with $0<|x|_{k} \leq 1$, there exists a $i$-cell $e \subset K$ such that $x \in e$. We then define $\Phi_{1}^{k}(x)=\Phi_{1}^{k}\left(x_{e}+\left(x-x_{e}\right) /|x|_{e}\right)$. In this way, we have defined $\Phi_{1}^{k}$ on $\left\{x \in K^{i}: 0<|x|_{k} \leq 1\right\}$. Thus by induction, $\Phi_{1}^{k}$ is defined on $\left\{x \in K: 0<|x|_{k} \leq 1\right\}$ such that $\Phi_{1}^{k}:\{x \in$ $\left.K: 0<|x|_{k} \leq 1\right\} \rightarrow \Gamma_{1}^{k}$ is a retraction. Next for $0<\epsilon<1$, we define $\Phi_{\epsilon}^{k}:\left\{x \in K: 0<|x|_{k}<1\right\} \rightarrow \Gamma_{\epsilon}^{k}$. For this, we define $\Phi^{k}:\{x \in$ $\left.K: 0<|x|_{k}<1\right\} \times(0,1) \rightarrow K$ as $\Phi^{k}(x, \epsilon)=x_{e}+\epsilon\left(x-x_{e}\right) /|x|_{e}$ for $x \in e$ and $\epsilon \in(0,1)$, where $e$ is a $(k+1)$-cell of $K$. This defines $\Phi^{k}$ on $\left\{x \in K^{k+1}: 0<|x|_{k}<1\right\} \times(0,1)$. Suppose inductively that $\Phi^{k}$ is defined on $\left\{x \in K^{i-1}: 0<|x|_{k}<1\right\} \times(0,1)$ for some $k+2 \leq i \leq m$. We then define for $i$-cell $e \subset K, \Phi^{k}(x, \epsilon)=x_{e}+\lambda\left(\Phi^{k}\left(x_{e}+\left(x-x_{e}\right) /|x|_{k}, \epsilon / \lambda\right)-x_{e}\right)$, where $\lambda=\lambda(x, \epsilon)=1-(1-\epsilon)\left(1-|x|_{e}\right) /\left(1-|x|_{k}\right)$. This defines $\Phi^{k}$ on $\left\{x \in K^{i}: 0<|x|_{k} \leq 1\right\}$ and by induction, $\Phi^{k}$ is defined on $\{x \in K: 0<$ $\left.|x|_{k}<1\right\} \times(0,1)$. We then set $\Phi_{\epsilon}^{k}(x)=\Phi^{k}(x, \epsilon)$. This defines a retraction $\Phi_{\epsilon}^{k}:\left\{x \in K: 0<|x|_{k}<1\right\} \rightarrow \Gamma_{\epsilon}^{k}$.

From $\Phi^{k}$, we define $\Psi_{\delta, \epsilon}^{k}: K \rightarrow K$ for $0<\delta<\epsilon \leq 1$ as follows:

$$
\Psi_{\delta, \epsilon}^{k}(x)= \begin{cases}x & \text { if } \epsilon \leq|x|_{k} \leq 1 \\ \Phi^{k}(x, \epsilon) & \text { if } \delta \leq|x|_{k} \leq \epsilon \\ \Phi^{k}\left(x, \delta^{-1} \epsilon|x|_{k}\right) & \text { if } 0<|x|_{k} \leq \delta \\ x & \text { if }|x|_{k}=0 .\end{cases}
$$

We also denote the compositions $M \stackrel{h^{-1}}{\longrightarrow} K \stackrel{\Psi_{\delta, \epsilon}^{k}}{\longrightarrow} K \stackrel{h}{\longrightarrow} M$ by $\Psi_{\delta, \epsilon}^{k}$. 
$|\cdot|_{k}$ and $\Psi_{\delta, \epsilon}^{k}$ have the following properties (see [41], [10]):

$$
\begin{aligned}
\mathcal{H}^{m}\left(\left\{x \in M:|x|_{k} \leq \epsilon\right\}\right) & \leq C \epsilon^{k+1}, \quad \mathcal{H}^{m-1}\left(\left\{x \in M:|x|_{k}=\epsilon\right\}\right) \leq C \epsilon^{k}, \\
C^{-1} \leq\left.|d| x\right|_{k} \mid & \leq C, \\
\left|d \Psi_{\delta, \epsilon}^{k}(x)\right| & \leq C \epsilon|x|_{k}^{-1} \quad \text { for } \delta \leq|x|_{k} \leq \epsilon, \\
\left|d \Psi_{\delta, \epsilon}^{k}(x)\right| & \leq C \epsilon \delta^{-1} \text { for }|x|_{k} \leq \delta, \\
\operatorname{Jac}\left(\left.\Phi^{k}(\cdot, \delta)\right|_{\Gamma_{\epsilon}^{k}}\right) & \leq C\left(\frac{\delta}{\epsilon}\right)^{k} .
\end{aligned}
$$

We are now ready to prove Theorem 6.1.

Proof of Theorem 6.1. Suppose $[P]_{[2 p]}=[Q]_{[2 p]}$. In the following, we set $k=[2 p], M_{\epsilon}^{k}:=\left\{x \in M:|x|_{k} \geq \epsilon\right\}$ and $|\cdot|=|\cdot|_{k}$ for simplicity of notation. Then $M_{\epsilon}^{k}$ is a deformation retract of $M^{k}$. ( $\Psi_{\epsilon, t}^{k}$ for $\epsilon \leq t \leq 1$ gives a deformation retraction). Thus in particular $M^{k}$ and $M_{\epsilon / 2}^{k}$ have the same homotopy type and we have from our assumption that $\left.\left.P\right|_{M_{\epsilon / 2}^{k}} \simeq Q\right|_{M_{\epsilon / 2}^{k}}$. Let $\varphi_{\epsilon / 2}:\left.\left.Q\right|_{M_{\epsilon / 2}^{k}} \stackrel{\sim}{\longrightarrow} P\right|_{M_{\epsilon / 2}^{k}}$ be a bundle isomorphism. We may assume that $\varphi_{\epsilon / 2}$ is smooth over the interior of $M_{\epsilon / 2}^{k}$. Let $A_{P} \in \mathcal{A}^{\infty}(P)$ and $A_{Q} \in \mathcal{A}^{\infty}(Q)$ be arbitrary connections. Choose a cut off function $\rho_{\epsilon} \in C^{\infty}(M)$ such that $\rho_{\epsilon}(x)=1$ for $|x| \leq \epsilon$ and $\rho_{\epsilon}=0$ for $|x| \geq 3 \epsilon / 2$. Since the space of connections on $\left.Q\right|_{M_{\epsilon / 2}^{k}}$ is an affine space modeled on $C^{\infty}\left(M_{\epsilon / 2}^{k}, \operatorname{Ad}(Q)\right)$, there exists $\alpha_{\epsilon / 2} \in C^{\infty}\left(M_{\epsilon / 2}^{k}, \operatorname{Ad}(Q)\right)$ such that

$$
\varphi_{\epsilon / 2}^{*}\left(A_{P}\right)=A_{Q}+\operatorname{pr}_{Q}^{*} \alpha_{\epsilon / 2},
$$

where $\operatorname{pr}_{Q}: Q \rightarrow M$ is the bundle projection.

Define $A_{Q, \epsilon}:=A_{Q}+\operatorname{pr}_{Q}^{*}\left(\rho_{\epsilon} \alpha_{\epsilon / 2}\right)$. This defines a connection on $Q$. We define the bundle $Q \#{ }_{\epsilon} P \rightarrow M$ by gluing $\left.Q\right|_{M_{\epsilon / 2}^{k}} \rightarrow M_{\epsilon / 2}^{k}$ and $\left.P\right|_{\{|x| \leq \epsilon\}} \rightarrow$ $\{|x| \leq \epsilon\}$ over $\{\epsilon / 2 \leq|x| \leq \epsilon\}$ via the bundle isomorphism $\varphi_{\epsilon / 2}$. Notice that $\varphi_{\epsilon / 2}:\left.\left.Q\right|_{M_{\epsilon / 2}^{k}} ^{\rightarrow} P\right|_{M_{\epsilon / 2}^{k}}$ and the identity isomorphism $\left.\left.P\right|_{\{|x| \leq \epsilon\}} \underset{\mathrm{id}}{\stackrel{\sim}{\longrightarrow}} P\right|_{\{|x| \leq \epsilon\}}$ are glued together over $\{\epsilon / 2 \leq|x| \leq \epsilon\}$ to give a bundle isomorphism $Q \#_{\epsilon} P \stackrel{\sim}{\longrightarrow} P$ over $M$. Two connections $A_{Q, \epsilon}$ and $A_{P}$ are also glued together over $\{\epsilon / 2 \leq|x| \leq \epsilon\}$ via $\varphi_{\epsilon / 2}$ to give a connection $A_{Q \#_{\epsilon} P}$ on $Q \#_{\epsilon} P$. Thus we have $A_{Q \#_{\epsilon} P}=A_{Q, \epsilon}$ over $M_{\epsilon / 2}^{k}$ and $A_{Q \#_{\epsilon} P}=A_{P}$ over $\{|x| \leq \epsilon\}$ with respect to suitable trivializations.

We consider the bundle $P_{\delta, \epsilon}:=\Psi_{\delta, 3 \epsilon / 2}^{k}{ }^{*}\left(Q \#_{\epsilon} P\right) \rightarrow M$. Since $\Psi_{\delta, 3 \epsilon / 2}^{k}$ is homotopic to the identity, we have $P_{\delta, \epsilon} \simeq P$. We also notice that for any $1<q<\infty, P_{\delta, \epsilon}$ is a bundle of class $W^{1, q}$ and a connection defined by $A_{\delta, \epsilon}:=$ $\Psi_{\delta, 3 \epsilon / 2}^{k}{ }^{*}\left(A_{Q \#_{\epsilon} P}\right)$ is a $\mathfrak{A}^{1, q}$-connection on $P_{\delta, \epsilon}$, i.e., $A_{\delta, \epsilon} \in L^{2 q}$ and $d A_{\delta, \epsilon} \in L^{q}$. 
We now estimate the energy $\int_{M}\left|F_{A_{\delta, \epsilon}}\right|^{p} \mathrm{dvol}_{M}$. We write

$\int_{M}\left|F_{A_{\delta, \epsilon}}\right|^{p} \operatorname{dvol}_{M}=\int_{|x| \geq 3 \epsilon / 2}\left|F_{A_{\delta, \epsilon}}\right|^{p} \operatorname{dvol}_{M}+\int_{|x| \leq 3 \epsilon / 2}\left|F_{A_{\delta, \epsilon}}\right|^{p} \operatorname{dvol}_{M}=I_{1}+I_{2}$.

Here

$$
I_{1}=\int_{|x| \geq 3 \epsilon / 2}\left|F_{A_{Q}}\right|^{p} \mathrm{dvol}_{M}
$$

and

$$
I_{2}=\int_{\delta \leq|x| \leq 3 \epsilon / 2}\left|F_{A_{\delta, \epsilon}}\right|^{p} \operatorname{dvol}_{M}+\int_{|x| \leq \delta}\left|F_{A_{\delta, \epsilon}}\right|^{p} \operatorname{dvol}_{M}=I_{2,1}+I_{2,2} .
$$

We have the following estimate:

$$
\begin{aligned}
I_{2,1} & \leq C \epsilon^{2 p} \int_{\delta \leq|x| \leq 3 \epsilon / 2}|x|^{-2 p}\left|F_{A_{Q}}\left(\Psi_{\delta, 3 \epsilon / 2}^{k}(x)\right)\right|^{p} \mathrm{dvol}_{M} \\
& \leq C\left\|F_{A_{Q}}\right\|_{L^{\infty}(M)}^{p} \epsilon^{2 p} \int_{\delta \leq|x| \leq 3 \epsilon / 2}|x|^{-2 p} \mathrm{~d} x \\
& \leq C\left\|F_{A_{Q}}\right\|_{L^{\infty}(M)}^{p} \epsilon^{2 p} \int_{\delta}^{3 \epsilon / 2} r^{-2 p} \mathcal{H}^{m-1}(\{|x|=r\}) \mathrm{d} r \\
& \leq C\left\|F_{A_{Q}}\right\|_{L^{\infty}(M)}^{p} \epsilon^{2 p} \int_{\delta}^{3 \epsilon / 2} r^{-2 p+[2 p]} \mathrm{d} r \\
& \leq C\left\|F_{A_{Q}}\right\|_{L^{\infty}(M)}^{p} \cdot \frac{\epsilon^{[2 p]+1}}{1-2 p+[2 p]},
\end{aligned}
$$

where we have used (6.4) in the first line, co-area formula in the third line and (6.2) in the fourth line.

$I_{2,2}$ is estimated as:

$$
\begin{aligned}
I_{2,2} & \leq C \epsilon^{2 p} \delta^{-2 p} \int_{|x| \leq \delta}\left|F_{A_{Q \# \epsilon} P}\right|^{p} \operatorname{dvol}_{M} \\
& \leq C \epsilon^{2 p} \delta^{-2 p}\left\|F_{A_{Q \# \epsilon P}}\right\|_{L^{\infty}(M)}^{p} \mathcal{H}^{m}(\{|x| \leq \delta\}) \\
& \leq C \epsilon^{2 p} \delta^{[2 p]+1-2 p}\left\|F_{A_{Q \# \epsilon} P}\right\|_{L^{\infty}(M)}^{p},
\end{aligned}
$$

where in the first line we have used (6.5) while in the second line we have used (6.2).

From (6.8), (6.9), (6.10) and (6.11) we obtain

$$
\begin{aligned}
\int_{M}\left|F_{A_{\delta, \epsilon}}\right|^{p} \operatorname{dvol}_{M} \leq & \int_{|x| \geq 3 \epsilon / 2}\left|F_{A_{Q}}\right|^{p} \operatorname{dvol}_{M}+C\left\|F_{A_{Q}}\right\|_{L^{\infty}(M)}^{p} \cdot \frac{\epsilon^{[2 p]+1}}{1-2 p+[2 p]} \\
& +C \epsilon^{2 p} \delta^{[2 p]+1-2 p}\left\|F_{A_{Q \#_{\epsilon} P}}\right\|_{L^{\infty}(M)}^{p} .
\end{aligned}
$$

Here we notice that $\left\|F_{A_{Q \# \epsilon} P}\right\|_{L^{\infty}(M)}$ depends on $\epsilon$ but does not depend on $\delta$. 
For any $\eta>0$, we first choose $A_{Q} \in \mathcal{A}(Q)$ such that

$$
\int_{M}\left|F_{A_{Q}}\right|^{p} \operatorname{dvol}_{M}<m_{p}(Q ; G)+\frac{\eta}{4} .
$$

We next choose $\epsilon>0$ such that

$$
\int_{|x| \leq 3 \epsilon / 2}\left|F_{A_{Q}}\right|^{p} \operatorname{dvol}_{M}<\frac{\eta}{4}, \quad C\left\|F_{A_{Q}}\right\|_{L^{\infty}(M)}^{p} \frac{\epsilon^{[2 p]+1}}{1-2 p+[2 p]}<\eta / 4 .
$$

For such a choice of $\epsilon>0$, we choose $\delta>0$ such that

$$
C\left\|F_{A_{Q \# \epsilon} P}\right\|_{L^{\infty}(M)}^{p} \epsilon^{2 p} \delta^{1-2 p+[2 p]}<\frac{\eta}{4} .
$$

For such $\epsilon$ and $\delta$, we have from (6.12)

$$
\int_{M}\left|F_{A_{\delta, \epsilon}}\right|^{p} \operatorname{dvol}_{M}<m_{p}(Q ; G)+\eta .
$$

To conclude $m_{p}(P ; G) \leq m(Q ; G)$ from $(6.16)$, we need to approximate $P_{\delta, \epsilon}$ and $A_{\delta, \epsilon}$ by smooth ones. But this follows from an approximation theorem proved in [13, Theorem 2.1]: We have proved in [13, Theorem 2.1] that any Sobolev bundle $P=\left\langle\left\{U_{\alpha}\right\},\left\{g_{\alpha \beta}\right\}\right\rangle$ of class $W^{1, q} \cap C^{0}$ and any $\mathfrak{A}^{1, q}(P)$ connection $A=\left\{A_{\alpha}\right\}$ on it can be approximated by smooth ones in the following sense: Passing to a refinement if necessary (we use the same $\left\{U_{\alpha}\right\}$ for simplicity of notation), there exist $C^{\infty}$-bundles $P_{n}=\left\langle\left\{U_{\alpha}\right\},\left\{g_{\alpha \beta}^{n}\right\}\right\rangle$ and $C^{\infty}$-connections $A_{n}=\left\{A_{\alpha ; n}\right\}$ such that $\left\|g_{\alpha \beta}^{n}-g_{\alpha \beta}\right\|_{W^{1, q}\left(U_{\alpha \beta}\right)} \rightarrow 0$ and

$$
\sum_{\alpha}\left\|A_{\alpha ; n}-A_{\alpha}\right\|_{L^{2 q}\left(U_{\alpha}\right)}+\left\|d A_{\alpha ; n}-d A_{\alpha}\right\|_{L^{q}\left(U_{\alpha}\right)} \rightarrow 0
$$

as $n \rightarrow \infty$. Therefore from (6.16), we conclude that $m_{p}(P ; G) \leq m_{p}(Q ; G)$ since $\eta>0$ is arbitrary. Reversing the roles of $P$ and $Q$, we also have $m_{p}(Q ; G) \leq m_{p}(P ; G)$. We thus obtain $m_{p}(P ; G)=m_{p}(Q ; G)$. This completes the proof.

As a corollary we have

Corollary 6.1 Suppose $P \rightarrow M$ is a principal $G$-bundle such that its restriction to $M^{[2 p]},\left.P\right|_{M^{[2 p]}} \rightarrow M^{[2 p]}$, is isomorphic to a flat bundle over $M^{[2 p]}$. Then we have $m_{p}(P ; G)=0$.

Proof. Under the assumption, we need to show that there exists a flat bundle $Q \rightarrow M$ such that $[P]_{[2 p]}=[Q]_{[2 p]}$. Since $\left.P\right|_{M^{[2 p]}}$ is flat, there exists a representation $\rho: \pi_{1}\left(M^{[2 p]}\right) \rightarrow G$ such that $P=\widetilde{M^{[2 p]}} \times{ }_{\rho} G$, where $\widetilde{M^{[2 p]}}$ 
is the universal covering of $M^{[2 p]}$ and $\pi_{1}(M)=\pi_{1}\left(M^{[2 p]}\right)$ acts on $\widetilde{M^{[2 p]}}$ as deck transformations. Since $\pi_{1}(M)=\pi_{1}\left(M^{[2 p]}\right), \rho$ naturally defines a representation $\rho^{\prime}: \pi_{1}(M) \rightarrow G$ and $Q:=\widetilde{M} \times{ }_{\rho^{\prime}} G$ is a flat bundle over $M$

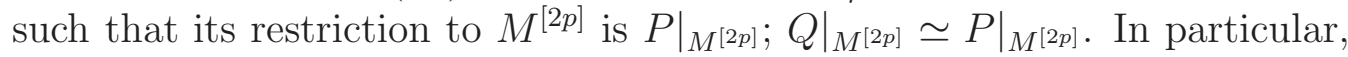
$[P]_{[2 p]}=[Q]_{[2 p]}$ and the assertion follows from Theorem 6.1.

Thus in particular, if $\left.P\right|_{M^{[2 p]}}$ is flat but $P$ itself is not, $m_{p}(P ; G)$ is never attained.

Example. The condition of Corollary 6.1 is satisfied if $\left.P\right|_{M^{[2 p]}}$ is the trivial flat bundle. This is the case if there exists $0 \leq j \leq[2 p]$ such that $\pi_{i}(M)=0$ for $0 \leq i \leq j$ and $\pi_{i}(G)=0$ for $j \leq i \leq[2 p]-1$. In fact, by the obstruction theory, the bundle $\left.P\right|_{M^{[2 p]}} \rightarrow M^{[2 p]}$ has a global section and it is trivial in such a case.

We conjecture that the converse of Corollary 6.1 is also true, i.e., if $m_{p}(P ; G)=0$, then $\left.P\right|_{M^{[2 p]}}$ is flat. At present, only partial results are proved, see the remark after Proposition 6.1 and Proposition 6.2 below.

As a special case $G=\mathbb{T}^{k}$, we have

Proposition 6.1 Assume $G=\mathbb{T}^{k}(k \geq 1)$ and $p>1$. Let $P \rightarrow M$ be a principal $G$-bundle. Then $m_{p}(P ; G)$ is always attained in the sense that there exist $0<\alpha<1$, a principal $G$-bundle $Q$ of class $C^{\infty}$ and a $C^{1, \alpha}$-connection $A$ on $Q$ such that $Q \simeq P$ and $m_{p}(P ; G)=\int_{M}\left|F_{A}\right|^{p} d v o l_{M}$.

Proof. Let $\left\{A_{n}\right\} \subset \mathcal{A}^{\infty}(P)$ be a minimizing sequence of $m_{p}(P ; G)$, i.e., $Y M_{p}\left(A_{n}\right) \rightarrow m_{p}(P ; G)$ as $n \rightarrow \infty$. Let $\left\{U_{\alpha}\right\}$ be an open covering of $M$ such that $U_{\alpha} \cong B^{m}$ and $U_{\alpha \beta} \cong B^{m}$ whenever $U_{\alpha \beta} \neq \emptyset$ (i.e., a "good" cover). We fix trivializations of $P$ over each $U_{\alpha}$ and with respect to these, we write $A_{n}=\left\{A_{\alpha ; n}\right\}$, where $A_{\alpha ; n} \in C^{\infty}\left(U_{\alpha}, T^{*} U_{\alpha} \otimes i \mathbb{R}^{k}\right)\left(i \mathbb{R}^{k}\right.$ is the Lie algebra of $\left.\mathbb{T}^{k}\right)$. We choose $g_{\alpha ; n}=\exp \left(f_{\alpha ; n}\right) \in C^{\infty}\left(U_{\alpha}, \mathbb{T}^{k}\right)\left(f_{\alpha ; n} \in C^{\infty}\left(U_{\alpha}, i \mathbb{R}^{k}\right)\right)$ such that

$$
d^{*}\left(d f_{\alpha ; n}+A_{\alpha ; n}\right)=0 \quad \text { in } U_{\alpha}
$$

and

$$
\iota_{\nu}\left(d f_{\alpha ; n}+A_{\alpha ; n}\right)=0 \quad \text { on } \partial U_{\alpha},
$$

where $\nu$ is the outward normal vector of $\partial U_{\alpha}$ in $M . f_{\alpha ; n}$ is uniquely determined up to constants. The gauge transformed connection

$$
A_{\alpha ; n}^{\prime}:=g_{\alpha ; n}^{-1} d g_{\alpha ; n}+A_{\alpha ; n}
$$

satisfies

$$
d^{*} A_{\alpha ; n}^{\prime}=0 \quad \text { in } U_{\alpha}, \quad \iota_{\nu} A_{\alpha ; n}^{\prime}=0 \quad \text { on } \partial U_{\alpha} .
$$


We may assume at the beginning that the trivializations of $P$ are chosen such that $A_{n}$ satisfies (6.19). By the elliptic estimate, we have from (6.19) that

$$
\left\|A_{\alpha ; n}\right\|_{W^{1, p}\left(U_{\alpha}\right)} \leq C\left\|d A_{\alpha ; n}\right\|_{L^{p}\left(U_{\alpha}\right)}=C\left\|F_{A_{n}}\right\|_{L^{p}\left(U_{\alpha}\right)},
$$

where $C>0$ is a constant independent of $n$.

Let $\left\{g_{\alpha \beta ; n}\right\}$ be the gluing cocycle of $P$ with respect to the trivialization as

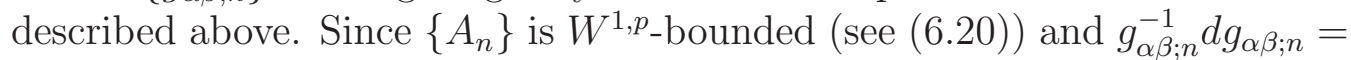
$A_{\beta ; n}-A_{\alpha ; n}$, it follows that $\left\{g_{\alpha \beta ; n}\right\}$ is $W^{2, p}$-bounded. We may assume (after passing to a subsequence if necessary) $\left\{A_{\alpha ; n}\right\}$ weakly converges to some $A_{\alpha} \in W^{1, p}\left(U_{\alpha} ; T^{*} U_{\alpha} \otimes i \mathbb{R}^{k}\right)$ in $W^{1, p}\left(U_{\alpha}\right)$.

Since $U_{\alpha \beta}$ is contractible, there exists a lift $\varphi_{\alpha \beta ; n} \in C^{\infty}\left(U_{\alpha \beta}, i \mathbb{R}^{k}\right)$ of $g_{\alpha \beta ; n}$, i.e., $g_{\alpha \beta ; n}=\exp \left(\varphi_{\alpha \beta ; n}\right)$. We write

$$
\varphi_{\alpha \beta ; n}=\varphi_{\alpha \beta ; n}^{\circ}+\bar{\varphi}_{\alpha \beta ; n}
$$

where $\bar{\varphi}_{\alpha \beta ; n}=\int_{U_{\alpha \beta}} \varphi_{\alpha \beta ; n} / \mathcal{H}^{m}\left(U_{\alpha \beta}\right) \in i \mathbb{R}^{k}$ is the mean value of $\varphi_{\alpha \beta ; n}$ over $U_{\alpha \beta}$. Define

$$
c_{\alpha \beta ; n}:=\bar{\varphi}_{\alpha \beta ; n}+2 \pi i \mathfrak{k}, \quad \text { where } \mathfrak{k}=-\left[\bar{\varphi}_{\alpha \beta ; n} / 2 \pi i\right] \in \mathbb{Z}^{k} .
$$

(For $a=\left(a_{1}, \ldots, a_{k}\right) \in \mathbb{R}^{k}$, we write $\left.[a]=\left(\left[a_{1}\right], \ldots,\left[a_{k}\right]\right) \in \mathbb{Z}^{k}\right)$. Then each component of $c_{\alpha \beta ; n} / i$ is in $[0,2 \pi)$ and $g_{\alpha \beta ; n}=\exp \left(\varphi_{\alpha \beta ; n}^{\circ}+c_{\alpha \beta ; n}\right)$. Since $A_{\alpha ; n}$ is in Coulomb gauge, we have

$$
\Delta \varphi_{\alpha \beta ; n}=0 \quad \text { in } U_{\alpha \beta}, \quad \int_{U_{\alpha \beta}} \varphi_{\alpha \beta ; n}^{\circ}=0 .
$$

Moreover, since $\left\{g_{\alpha \beta ; n}\right\}$ is $W^{2, p}$-bounded, $d \varphi_{\alpha \beta ; n}^{\circ}$ and $\nabla d \varphi_{\alpha \beta ; n}^{\circ}$ are bounded in $L^{p}\left(U_{\alpha \beta}\right)$ and thus $\varphi_{\alpha \beta ; n}^{\circ}$ is bounded in $W^{2, p}\left(U_{\alpha \beta}\right)$ by the Poincaré inequality. Therefore from (6.21) and the boundedness of $\left\{c_{\alpha \beta ; n}\right\}$, there exists a subsequence (which we still denote by the same sequence) and $\varphi_{\alpha \beta} \in C^{\infty}\left(U_{\alpha \beta}, i \mathbb{R}^{k}\right)$ such that

$$
\varphi_{\alpha \beta ; n}^{\circ}+c_{\alpha \beta ; n} \rightarrow \varphi_{\alpha \beta} \quad \text { in } C_{\mathrm{loc}}^{\infty}\left(U_{\alpha \beta}\right) .
$$

Define $g_{\alpha \beta}:=\exp \left(\varphi_{\alpha \beta}\right)$. Then

$$
g_{\alpha \beta ; n} \rightarrow g_{\alpha \beta} \quad \text { in } C_{\mathrm{loc}}^{\infty}\left(U_{\alpha \beta}\right)
$$

and a $G$-bundle defined by $P_{\infty}:=\left\langle\left\{U_{\alpha}\right\},\left\{g_{\alpha \beta}\right\}\right\rangle$ is isomorphic to $P$. By the lower semi-continuity of the integral $\int_{M}\left|F_{A_{n}}\right|^{p} \mathrm{dvol}_{M}$, we have

$$
\int_{M}\left|F_{A}\right|^{p} \operatorname{dvol}_{M} \leq m_{p}(P ; G) \text {. }
$$

Therefore $m_{p}(P ; G)$ is attained.

Since the limit connection $A_{\infty}:=\left\{A_{\alpha}\right\}$ is in Coulomb gauge, the regularity of the limit connection $A_{\infty}$ follows from [37] and [11]. This completes the proof. 
Thus in particular, if $m_{p}\left(P ; \mathbb{T}^{k}\right)=0, P$ is flat. For general $G$, we only have the following partial converse of Corollary 6.1:

Proposition 6.2 Suppose that $m_{p}(P ; G)=0$ for a compact Lie group $G$ and a smooth principal $G$-bundle $P \rightarrow M$. Then we have:

(1) If $M$ is $([2 p]-1)$-connected (i.e., $\pi_{i}(M)=0$ for $\left.1 \leq i \leq[2 p]-1\right)$, then $[P]_{[2 p]}=0$.

(2) For any $\phi \in I^{k}(G)$ with $2 k \leq[2 p]$, we have $P_{\phi}(P)=0$ in $H^{2 k}(M ; \mathbb{R})$.

Proof. Let $h: K \rightarrow M$ be a Lipschitz triangulation of $M$ and $\varphi: S^{[2 p]} \rightarrow K^{[2 p]}$ any Lipschitz map. We claim that for a.e. $v \in \mathbb{B}_{\epsilon}^{l}$, we have $m_{p}\left(\left(h_{v} \circ\right.\right.$ $\left.\varphi)^{*} P ; G\right)=0$. To see this, choose $\left\{A_{n}\right\} \subset \mathcal{A}^{\infty}(P)$ such that $m_{p}(P ; G)=$ $\lim _{n \rightarrow \infty} \int_{M}\left|F_{A_{n}}\right|^{p} \operatorname{dvol}_{M}=0$. By Fubini's theorem, we have

$$
\begin{aligned}
\int_{\mathbb{B}_{\epsilon}^{l}}\left(\int_{S^{[2 p]}}\left|F_{\left(h_{v} \circ \varphi\right) * A_{n}}\right|^{p} \operatorname{dvol}_{S^{[2 p]}}\right) \mathrm{d} \mathcal{H}^{l}(v) \\
\quad \leq C_{\varphi} \int_{S^{[2 p]}}\left(\int_{\mathbb{B}_{\epsilon}^{l}}\left|F_{A_{n}}\left(\pi_{M}(h(\varphi(x))+v)\right)\right|^{p} \mathrm{~d} \mathcal{H}^{l}(v)\right) \mathrm{dvol}_{S^{[2 p]}} \\
\quad \leq C_{\varphi} \int_{M}\left|F_{A_{n}}\right|^{p} \operatorname{dvol}_{M} .
\end{aligned}
$$

By (6.22) and Fatou's lemma, we have $\liminf _{n \rightarrow \infty} \int_{S^{[2 p]}}\left|F_{\left(h_{v} \circ \varphi\right) * A_{n}}\right|^{p} \operatorname{dvol}_{S^{[2 p]}}=0$ for a.e. $v \in \mathbb{B}_{\epsilon}^{l}$. From this, we have $m_{p}\left(\left(h_{v} \circ \varphi\right)^{*} P ; G\right)=0$ for a.e $v \in \mathbb{B}_{\epsilon}^{l}$. From [13, Corollary 5.1], we deduce that $\left(h_{v} \circ \varphi\right)^{*} P \rightarrow S^{[2 p]}$ is isomorphic to the trivial bundle.

We now consider the case (1). Since $M$ is $([2 p]-1)$-connected, there exists a CW-complex $L$ such that $L$ is homotopy equivalent to $M$ and $L^{[2 p]-1}=*$. Thus in particular, $L^{[2 p]}=\bigvee_{\sigma} S_{\sigma}^{[2 p]}\left(S_{\sigma}^{[2 p]}\right.$ is a copy of $\left.S^{[2 p]}\right)$. Let $c_{P}: M \rightarrow B G$ be the classifying map of $P$, i.e., $P \simeq c_{P}^{*}(E G)$, where $E G \rightarrow B G$ is the universal $G$-bundle. By the claim proved in the previous paragraph, $c_{P} \circ h_{v} \circ \varphi$ is homotopically trivial for a homotopy equivalence $\varphi: L^{[2 p]}=\bigvee_{\sigma} S_{\sigma}^{[2 p]} \stackrel{\sim}{\longrightarrow} K^{[2 p]}$. Thus $c_{P} \circ h_{v}: K^{[2 p]} \rightarrow B G$ is also homotopically trivial and $\left.h_{v}^{*} P\right|_{K^{[2 p]}}$ is isomorphic to the trivial bundle. Therefore we have $[P]_{[2 p]}=0$. This completes the proof of $(1)$.

To prove (2), as the proof of the above claim, for any $2 k$-dimensional submanifold $S \subset M$, we have $\left.P\right|_{S}$ is flat (see [13, Lemma 5.1]) and $\left\langle P_{\phi}(P),[S]\right\rangle=$ $\left\langle\left[P_{\phi}(A)\right],[S]\right\rangle$ for any $A \in \mathcal{A}^{\infty}\left(\left.P\right|_{S}\right)$. Since $\left.P\right|_{S} \rightarrow S$ has a flat connection, we thus have $\left\langle P_{\phi}(P),[S]\right\rangle=0$. Since $H_{*}(M ; \mathbb{Q}) \cong \Omega_{*}(M) \otimes \mathbb{Q}$, this implies $P_{\phi}(P)=0$. This completes the proof of $(2)$. 


\section{Appendix}

In this appendix, we prove two results: An approximation theorem of Sobolev bundles defined over polyhedron and the intersection lemma, Lemma 5.1.

\subsection{An approximation theorem of Sobolev bundles over polyhe- dron}

We extend the approximation theorem and other properties of Sobolev bundles proved in $[13, \S 3]$ for bundles over polyhedron. We only indicate needed modifications and please refer to $[13, \S 3]$ for details.

We assume throughout this subsection that $K$ is an $m$-dimensional finite regular polyhedron (we do not distinguish between a simplicial complex and the polyhedron defined from it). It is known that polyhedron is ENR, see [5, Appendix E]. Thus we may assume that $K \subset \mathcal{O} \subset \mathbb{R}^{l}$ for some open set $\mathcal{O} \subset \mathbb{R}^{l}$ and $K$ is a retract of $\mathcal{O}$. We further assume that there exists a Lipschitz retraction $\pi_{K}: \mathcal{O} \rightarrow K$ such that

(A-1) there exists $C_{1}>0$ such that $C_{1}^{-1} \leq \mathcal{H}^{l-m}\left(\pi_{K}^{-1}(y)\right) \leq C_{1}$ for all $y \in K$,

(A-2) there exists $C_{2}>0$ such that $C_{2}^{-1} \leq \operatorname{Jac}\left(\pi_{K}\right)(x) \leq C_{2}$ for a.e. $x$, where $\operatorname{Jac}\left(\pi_{K}\right)$ is the $m$-dimensional jacobian of $\pi_{K}$.

The precise meaning of (A-2) is as follows: For each $m$-simplex $\Delta$ of $K$, consider $\pi_{K, \Delta}:=\left.\pi_{K}\right|_{\pi_{K}^{-1}(\Delta)}: \pi_{K}^{-1}(\Delta) \rightarrow \Delta$. (A-2) means that for each such $\Delta$, the $m$-dimensional jacobian, defined by $\operatorname{Jac}\left(\pi_{K, \Delta}\right)=\left(\operatorname{det}\left(d \pi_{K, \Delta} \circ\right.\right.$ $\left.\left.\left(d \pi_{K, \Delta}\right)^{t}\right)\right)^{1 / 2}$, satisfies $C_{2}^{-1} \leq \operatorname{Jac}\left(\pi_{K, \Delta}\right)(x) \leq C_{2}$ for a.e. $x \in \Delta$.

(A-1) and (A-2) are satisfied for $K$ the $m$-skeleton of a triangulated smooth manifold. To see this, we assume $\operatorname{dim} M=m+m_{1}, K=M^{m}$ and $M \subset \mathbb{R}^{l}$ for $l=m+m_{1}+m_{2}$. For $0<\epsilon<1$, set $K_{\epsilon}=\left\{x \in M:|x|_{m} \geq \epsilon\right\}$, where $|\cdot|_{m}$ is as defined in $\S 6$. Then $\Phi(\cdot, 1): K_{\epsilon} \rightarrow K$ as defined in $\S 6$ gives a Lipschitz retraction of $K_{\epsilon}$ onto $K$. Let $\pi_{M}: \mathcal{O}(M) \rightarrow M$ be a (smooth) retraction, where $\mathcal{O}(M) \subset \mathbb{R}^{l}$ is a tubular neighborhood of $M$ in $\mathbb{R}^{l}$. Define $\mathcal{O}=\pi_{M}^{-1}\left(K_{\epsilon}\right)$ and consider

$$
\pi_{K}: \mathcal{O} \stackrel{\pi_{M}}{\longrightarrow} K_{\epsilon} \stackrel{\Phi(\cdot, 1)}{\longrightarrow} K .
$$

Then $\pi_{K}$ is a Lipschitz neighborhood retract. For each $m$-simplex $\Delta, \pi_{K}$ on $\pi_{K}^{-1}(\Delta)$ is bi-Lipschitz equivalent to the composition

$$
x=\left(x_{m}, x_{m_{1}}, x_{m_{2}}\right) \mapsto\left(x_{m}, x_{m_{1}}\right) \mapsto x_{m},
$$

where $x_{m} \in \mathbb{R}^{m}, x_{m_{1}} \in \mathbb{R}^{m_{1}}$ and $x_{m_{2}} \in \mathbb{R}^{m_{2}}$. From this it is easy to see that (A-1) and (A-2) are satisfied. 
We show by a slight modification of the argument in $[13, \S 3]$ that the results obtained in [13, §3] (in particular Corollary 3.1) continue to hold for Sobolev bundles over finite regular polyhedron under assumptions (A-1) and (A-2).

Sobolev spaces on regular polyhedron are defined for example in [41]. The definition of Sobolev bundles over regular polyhedrons is the same as that of Sobolev bundles over smooth manifolds. The proof of the approximation theorem, i.e., the proof of the assertion that any Sobolev bundle of class $W^{1, m}$ is approximated by bundles of the class $C^{0} \cap W^{1, m}$ (in the sense as presented in $[13, \S 3])$ is the same as that of the smooth base manifold case, see $[13, \S 3]$. We need a slight modification of the argument for the proof of the stability assertion [13, Proposition 3.1]. The only needed modification is in the proof of [13, Lemma 3.1]. In that proof (see in particular the construction (3.22) in [13]) we have used collar neighborhood theorem for smooth submanifolds of $M$ which does not hold for the present case. Once the construction of (3.22) in [13] is modified, the other arguments go through for the present case.

We now give a modification of the argument. Fix an arbitrary $\eta>0$ such that $d(\partial \mathcal{O}, K) \geq 2 \eta$. Let $K^{\prime}$ be a subdivision of $K$ such that for any $m$-simplex $\triangle^{m}$ of $K^{\prime}$ there holds $\operatorname{diam}\left(\Delta^{m}\right)<\eta$. The set of all the $m$-simplexes of $K^{\prime}$ is denoted by $\left\{\triangle_{\alpha}\right\}_{\alpha \in A}$. Thus we have $|K|=\left|K^{\prime}\right|=$ $\bigcup_{\alpha \in A} \triangle_{\alpha}$. Taking $\eta>0$ small if necessary, we may assume that for the set $\tilde{\mathcal{O}}:=\{x \in \mathcal{O}: d(x, K)<\eta\}$, the restriction of $\pi_{K}$ to $\tilde{\mathcal{O}}$, denoted also by $\pi_{K}: \tilde{\mathcal{O}} \rightarrow K$ satisfies conditions (A-1) and (A-2) above. For $\xi \in \mathbb{R}^{l}$ with $|\xi|<\eta_{\sim}$ and an $m$-simplex $\triangle_{\alpha}$, set $\tilde{\triangle}_{\alpha, \xi}=\tilde{\triangle}_{\alpha}+\xi$ and $\triangle_{\alpha, \xi}:=\pi_{K}\left(\tilde{\triangle}_{\alpha, \xi}\right)$, where $\tilde{\triangle}_{\alpha}=\pi_{K}^{-1}\left(\triangle_{\alpha}\right)$. For $U \subset K$ an open subset and $f \in L^{1}(U)$, we first remark that the following inequality holds:

$$
\int_{|\xi|<\eta}\left(\int_{\partial \triangle_{\alpha, \xi} \cap U} f(x) d \mathcal{H}^{m-1}(x)\right) d \mathcal{H}^{l}(\xi) \leq C \int_{U} f(x) d \mathcal{H}^{m}(x) .
$$

To prove (7.1), we first have

$$
\begin{aligned}
& \int_{|\xi|<\eta}\left(\int_{\pi_{K}^{-1}\left(\partial \triangle_{\alpha, \xi} \cap U\right)} f \circ \pi_{K}(x) d \mathcal{H}^{l-1}(x)\right) d \mathcal{H}(\xi) \\
& 2) \quad C_{2}^{-1} \int_{|\xi|<\eta}\left(\int_{\pi_{K}^{-1}\left(\partial \triangle_{\alpha, \xi} \cap U\right)} f \circ \pi_{K}(x) \operatorname{Jac}\left(\pi_{K}\right)(x) d \mathcal{H}^{l-1}(x)\right) d \mathcal{H}^{l}(\xi) \\
& 3) \geq C_{2}^{-1} \int_{|\xi|<\eta}\left(\int_{\partial \triangle_{\alpha, \xi} \cap U} f(y) \mathcal{H}^{l-m}\left(\pi_{K}^{-1}(y)\right) d \mathcal{H}^{m-1}(y)\right) d \mathcal{H}^{l}(\xi) \\
& 4 C_{2}^{-1} C_{1}^{-1} \int_{|\xi|<\eta}\left(\int_{\partial \triangle_{\alpha, \xi} \cap U} f(y) d \mathcal{H}^{m-1}(y)\right) d \mathcal{H}^{l}(\xi),
\end{aligned}
$$


where we have used (A-2) to derive (7.2), (7.3) is obtained by applying the co-area formula [29] and (7.4) is by (A-1).

On the other hand, we have

$$
\begin{aligned}
\int_{|\xi|<\eta} & \left(\int_{\pi_{K}^{-1}\left(\partial \triangle_{\alpha, \xi} \cap U\right)} f \circ \pi_{K}(x) d \mathcal{H}^{l-1}(x)\right) d \mathcal{H}^{l}(\xi) \\
= & \int_{|\xi|<\eta}\left(\int_{\partial \tilde{\Delta}_{\alpha, \xi} \cap \tilde{U}} f \circ \pi_{K}(x) d \mathcal{H}^{l-1}(x)\right) d \mathcal{H}^{l}(\xi)\left(\tilde{U}:=\pi_{K}^{-1}(U)\right) \\
= & \int_{|\xi|<\eta}\left(\int_{\partial \tilde{\Delta}_{\alpha} \cap(\tilde{U}-\xi)} f \circ \pi_{K}(x+\xi) d \mathcal{H}^{l-1}(x)\right) d \mathcal{H}^{l}(\xi) \\
= & \int_{\partial \tilde{\Delta}_{\alpha}}\left(\int_{\tilde{U} \cap B_{\eta}^{l}(x)} f \circ \pi_{K}(z) d \mathcal{H}^{l}(z)\right) d \mathcal{H}^{l-1}(x) \\
\leq & \int_{\partial \tilde{\triangle}_{\alpha}}\left(\int_{\tilde{U}} f \circ \pi_{K}(z) d \mathcal{H}^{l}(z)\right) d \mathcal{H}^{l-1}(x) \\
\leq & C_{A} \int_{\tilde{U}} f \circ \pi_{K}(z) d \mathcal{H}^{l}(z) \\
\leq & C_{A} C_{2} \int_{\tilde{U}} f \circ \pi_{K}(z) \operatorname{Jac}^{l}\left(\pi_{K}\right)(z) d \mathcal{H}^{l}(z) \\
\leq & C_{A} C_{2} \int_{U} f(y) \mathcal{H}^{l-m}\left(\pi_{K}^{-1}(y)\right) d \mathcal{H}^{m}(y) \\
\leq & C_{A} C_{2} C_{1} \int_{U} f(x) d \mathcal{H}^{m}(x),
\end{aligned}
$$

where $C_{A}$ in (7.5) is defined by $C_{A}=\sup _{\alpha \in A} \mathcal{H}^{l-1}\left(\partial \widetilde{\triangle}_{\alpha}\right)$, (7.6) is derived by (A-2), (7.7) is obtained by applying the co-area formula and (7.8) is by $(\mathrm{A}-1)$.

Combining (7.4) and (7.8), we obtain (7.1) with $C=C_{A} C_{1}^{2} C_{2}^{2}$.

We apply (7.1) for

$$
U=\left(\bigcup_{\alpha \leq k} V_{\alpha}^{k}\right) \cap V_{k+1}
$$

and

$$
f=\left|\rho_{k+1}^{\epsilon}-\mathbf{1}\right| \quad \text { or } \quad f=\left|g_{\alpha \beta}^{1, \epsilon}-h_{\alpha \beta}^{0, \epsilon}\right|,
$$

where $V_{\alpha}^{k}, V_{k+1}, \rho_{k+1}^{\epsilon}, g_{\alpha \beta}^{1, \epsilon}$ and $h_{\alpha \beta}^{0, \epsilon}$ are as defined in the proof of Lemma 3.1 in [13]. Then by Fubini's theorem, there exist a sequence of positive numbers $\left\{\epsilon_{k}\right\}$ converging to 0 and $\xi \in \mathbb{R}^{l}$ with $|\xi|<\eta$ such that

$$
\begin{aligned}
& \left\|\rho_{k+1}^{\alpha}-\mathbf{1}\right\|_{W^{1, m}\left(\cup_{\alpha} \partial \triangle_{\alpha, \xi} \cap U\right)} \rightarrow 0 \quad \text { and } \\
& \left\|h_{\alpha \beta}^{1, \epsilon_{k}}-g_{\alpha \beta}^{0, \epsilon_{k}}\right\|_{W^{1, m}\left(\cup_{\alpha} \partial \triangle_{\alpha, \xi} \cap U\right)} \rightarrow 0
\end{aligned}
$$

as $k \rightarrow \infty$. 
We then define

$V^{k+1}:=U \cap \bigcup_{\alpha \in A}\left\{\triangle_{\alpha, \xi}: \triangle_{\alpha, \xi} \cap U \neq \emptyset, \triangle_{\alpha, \xi} \cap \partial\left(\bigcup_{\alpha \leq k} V_{\alpha}^{k} \cap V_{k+1}\right) \cap V_{k+1}=\emptyset\right\}$

Since $\partial V^{k+1} \cap U \subset \bigcup_{\alpha} \partial \triangle_{\alpha, \xi} \cap U$, we have from (7.9) and (7.10) that

$$
\left\|\rho_{k+1}^{\alpha}-\mathbf{1}\right\|_{W^{1, m}\left(\partial V^{k+1} \cap\left(\cup_{\alpha} V_{\alpha}^{k} \cap V_{k+1}\right)\right)} \rightarrow 0
$$

and

$$
\left\|h_{\alpha \beta}^{1, \epsilon_{k}}-g_{\alpha \beta}^{0, \epsilon_{k}}\right\|_{W^{1, m}\left(\partial V^{k+1} \cap\left(\cup_{\alpha} V_{\alpha}^{k} \cap V_{k+1}\right)\right)} \rightarrow 0
$$

as $k \rightarrow \infty$.

From here, the remaining argument is the same as in the smooth base manifold case. Namely, from (7.11) and the Sobolev embedding theorem, we can write

$$
\rho_{k+1}^{\epsilon}=\exp \left(X_{k+1}^{\epsilon}\right)
$$

on $\partial V^{k+1} \cap \bigcup_{\alpha \leq k} V_{\alpha}^{k} \cap V_{k+1}$, where exp $: \mathfrak{g} \rightarrow G$ is the exponential map,

$$
\left\|X_{k+1}^{\epsilon}\right\|_{L^{\infty}} \rightarrow 0
$$

and

$$
\left\|X_{k+1}^{\epsilon}\right\|_{W^{1, m}} \rightarrow 0
$$

as $k \rightarrow \infty\left(L^{\infty}\right.$ and $W^{1, m}$-norms are taken on $\left.\partial V^{k+1} \cap \bigcup_{\alpha \leq k} V_{\alpha}^{k} \cap V_{k+1}\right)$.

Since

$$
\partial V^{k+1} \cap V_{k+1} \subset \bigcup_{\alpha} \partial \triangle_{\alpha, \xi} \cap V_{k+1}
$$

and $\bigcup_{\alpha} \partial \triangle_{\alpha, \xi}$ is a Lipschitz deformation retract of $\bigcup_{\alpha}\left(\triangle_{\alpha, \xi} \backslash\left\{c_{\alpha}\right\}\right)\left(c_{\alpha}\right.$ is the barycenter of $\left.\triangle_{\alpha, \xi}\right), X_{k+1}^{\epsilon}$ can be extended to a neighborhood of $\partial V^{k+1} \cap V_{k+1}$ in $V_{k+1}$ as a $W^{1, m}$-function which we denote as $\tilde{X}_{k+1}^{\epsilon}$. Let $\phi$ be a Lipschitz function such that its support is contained in that neighborhood and $\phi=1$ on another smaller neighborhood of $\partial V^{k+1} \cap V_{k+1}$ in $V_{k+1}$. Define $\tilde{\rho}_{k+1}^{\epsilon}$ such that $\tilde{\rho}_{k+1}^{\epsilon}=\rho_{k+1}^{\epsilon}$ in $V^{k+1}$ and $\tilde{\rho}_{k+1}^{\epsilon}=\exp \left(\phi \tilde{X}_{k+1}^{\epsilon}\right)$ in $V_{k+1} \backslash \overline{V^{k+1}}$. For this choice of $\tilde{\rho}_{k+1}^{\epsilon}$, the argument of [13] goes through and this completes the proof. 


\subsection{Proof of the intersection lemma}

We prove Lemma 5.1 in $\S 5$.

Proof of Lemma 5.1. We first assume that $S \subset M$ is closed with $\mathcal{H}^{m-s}(S)<+\infty$ and $h: K \rightarrow M$ is smooth, i.e., its restriction to each $d$ cell $\triangle^{d}$ is smooth in the sense that $\left.h\right|_{\triangle^{d}}$ has a smooth extension to an open neighborhood of $\triangle^{d}$ in the $d$-plane containing $\triangle^{d}$. Define $F: \mathbb{B}_{\epsilon} \times K \times M \rightarrow$ $M \times M$ by $F(v, x, y)=\left(h_{v}(x), y\right)$, where $h_{v}(x)=\pi_{M}(h(x)+v)$. For $\triangle^{d}$ a $d$-cell of $K$, define $F_{\triangle^{d}}=\left.F\right|_{\mathbb{B}_{\epsilon}^{l} \times \triangle^{d} \times M}$. It is easy to see that $F_{\triangle^{d}}$ intersects transversally to the diagonal $\Delta \subset M \times M$. In fact, we have $d F_{\triangle^{d}}(v, x, y)(V, 0, Y)=\left(d \pi_{M}(h(x)+v)(V), Y\right)$ for $(v, x, y) \in \mathbb{B}_{\epsilon}^{l} \times \triangle^{d} \times M$, $V \in T_{v} \mathbb{B}_{\epsilon}^{l}=\mathbb{R}^{l}$ and $Y \in T_{y} M$ and this is onto as a map from $T_{v} \mathbb{B}_{\epsilon}^{l} \times T_{y} M$ to $T_{\left(h_{v}(x), y\right)}(M \times M)$. Thus by the standard transversality theorem, we see that $F_{\triangle^{d}}^{-1}(\Delta) \subset \mathbb{B}_{\epsilon}^{l} \times \triangle^{d} \times M$ is a $(l+d)$-dimensional submanifold and $\mathcal{H}^{l+d}\left(F_{\triangle^{d}}^{-1}(\Delta)\right)<+\infty$. Since $F^{-1}(\Delta)=\bigcup_{\triangle^{d}} F_{\triangle^{d}}^{-1}(\Delta)$, we also have

$$
\mathcal{H}^{l+d}\left(F^{-1}(\Delta)\right)<+\infty \text {. }
$$

We next consider the set $B:=\mathbb{B}_{\epsilon}^{l} \times K \times S$. For $u \in \mathbb{B}_{\epsilon}^{l}$, define $B_{u}=\mathbb{B}_{\epsilon}^{l} \times$ $K \times S_{u}$, where $S_{u}=\pi_{M}(S+u)$. We consider the intersection $F^{-1}(\Delta) \cap B_{u}$. We claim that $\operatorname{dim}\left(F^{-1}(\Delta) \cap B_{u}\right) \leq l+d-s$ for a.e. $u \in \mathbb{B}_{\epsilon}^{l}$. To prove this claim, we consider the map $F: \mathbb{B}_{\epsilon}^{l} \times K \times \mathcal{O}(M) \rightarrow M \times M$ defined by $\tilde{F}(v, x, y)=\left(h_{v}(x), \pi_{M}(y)\right)$, where we set $\mathcal{O}(M)=\mathcal{O}_{\epsilon / 2}(M)$. We also define $\hat{F}: \mathbb{B}_{\epsilon}^{l} \times K \times \mathcal{O}(M) \times S \rightarrow M \times M$ by $\hat{F}(v, x, y, z)=\left(h_{v}(x), \pi_{M}(y)\right)$ and $P_{1,2,3}: \mathbb{B}_{\epsilon}^{l} \times K \times \mathcal{O}(M) \times S \rightarrow \mathbb{B}_{\epsilon}^{l} \times K \times \mathcal{O}(M)$ by $P_{1,2,3}(v, x, y, z)=(v, x, y)$. As in the case of $F, \tilde{F}$ transversally intersects with $\Delta$ and we have

$$
\mathcal{H}^{2 l+d-m}\left(\tilde{F}^{-1}(\Delta)\right)<+\infty
$$

and hence we also have

$$
\mathcal{H}^{2 l+d-s}\left(\hat{F}^{-1}(\Delta)\right)<+\infty
$$

since $\hat{F}^{-1}(\Delta)=\tilde{F}^{-1}(\Delta) \times S$ and $\tilde{F}^{-1}(\Delta)$ is a piecewise smooth manifold (i.e., a finite union of manifolds of the form $\left.\tilde{F}_{\triangle d}^{-1}(\Delta)\right)$, so the result of $[8,2.10 .45]$ is applicable and we have

$$
\mathcal{H}^{2 l+d-s}\left(\hat{F}^{-1}(\Delta)\right) \leq C \mathcal{H}^{2 l+d-m}\left(\tilde{F}^{-1}(\Delta)\right) \mathcal{H}^{m-s}(S)<+\infty .
$$

For the set $V_{u}:=\left\{(v, x, y, z) \in \mathbb{B}_{\epsilon}^{l} \times K \times \mathcal{O}(M) \times \mathcal{O}(M): y-z=u\right\}$ defined for $u \in \mathbb{B}_{\epsilon}^{l}$, we have

$$
\begin{aligned}
\hat{F}^{-1}(\Delta) \cap V_{u} & =\left\{(v, x, z+u, z) \in \tilde{F}^{-1}(\Delta) \times S\right\} \\
& =\hat{F}^{-1}(\Delta) \cap p^{-1}(u)
\end{aligned}
$$

where $p: \mathbb{B}_{\epsilon}^{l} \times K \times \mathcal{O}(M) \times \mathcal{O}(M) \rightarrow \mathbb{B}_{\epsilon}^{l}$ is defined by $p(v, x, y, z)=y-z$. 
By the co-area formula (see [19, Theorem 7.7]), we have

$$
\int_{\mathbb{B}_{\epsilon}^{l}} \mathcal{H}^{l+d-s}\left(\hat{F}^{-1}(\Delta) \cap p^{-1}(u)\right) \mathrm{d} \mathcal{H}^{l}(u) \leq C \mathcal{H}^{2 l+d-s}\left(\hat{F}^{-1}(\Delta)\right)<+\infty .
$$

Therefore for a.e. $u \in \mathbb{B}_{\epsilon}^{l}$, we obtain

$$
\mathcal{H}^{l+d-s}\left(\hat{F}^{-1}(\Delta) \cap p^{-1}(u)\right)<+\infty .
$$

Notice here that $\hat{F}^{-1}(\Delta) \cap p^{-1}(u)$ is given as

$$
\begin{aligned}
\hat{F}^{-1}(\Delta) & \cap p^{-1}(u)= \\
= & \left\{(v, x, z+u, z) \in \mathbb{B}_{\epsilon}^{l} \times K \times \mathcal{O}(M) \times S: h_{v}(x)=\iota_{u}(z)\right\} .
\end{aligned}
$$

We consider the image of this set under the projection

$$
P_{1,2,4}: \mathbb{B}_{\epsilon}^{l} \times K \times \mathcal{O}(M) \times S \ni(v, x, y, z) \mapsto(v, x, z) \in \mathbb{B}_{\epsilon}^{l} \times K \times S .
$$

We denote this by $A_{u}:=P_{1,2,4}\left(\hat{F}^{-1}(\Delta) \cap p^{-1}(u)\right)$. From (7.21), $A_{u}$ is given by

$$
A_{u}=\left\{(v, x, z) \in \mathbb{B}_{\epsilon}^{l} \times K \times S: h_{v}(x)=\iota_{u}(z)\right\}
$$

and since this is a Lipschitz image of $\hat{F}^{-1}(\Delta) \cap p^{-1}(u)$ which satisfies (7.20), we have $\mathcal{H}^{l+d-s}\left(A_{u}\right)<+\infty$ for a.e. $u \in \mathbb{B}_{\epsilon}^{l}$. Thus, in particular, we have $\mathcal{H}-$ $\operatorname{dim} A_{u} \leq l+d-s$. Since the map $A_{u} \ni(v, x, z) \mapsto\left(v, x, \iota_{u}(z)\right) \in F^{-1}(\Delta) \cap B_{u}$ is bi-Lipschitz, we also have $\mathcal{H}-\operatorname{dim}\left(F^{-1}(\Delta) \cap B_{u}\right) \leq l+d-s$ for a.e. $u \in \mathbb{B}_{\epsilon}^{l}$. Thus the claim is proved.

We return to the proof of the lemma. Consider a.e $u \in \mathbb{B}_{\epsilon}^{l}$ such that $\operatorname{dim}\left(F^{-1}(\Delta) \cap B_{u}\right) \leq l+d-s$. Let $P_{1}: F^{-1}(\Delta) \cap B_{u} \rightarrow \mathbb{B}_{\epsilon}^{l}$ be the projection to the first factor. Then we have

$$
P_{1}^{-1}(v)=\left\{\left(x, h_{v}(x)\right) \in K \times M: \iota_{u}^{-1} \circ h_{v}(x) \in S\right\} .
$$

Thus to complete the proof of the lemma for the case $\mathcal{H}^{m-s}(S)<+\infty$, we need to show that for a.e. $v \in \mathbb{B}_{\epsilon}^{l}$, we have $P_{1}^{-1}(v)=\emptyset$. To prove this, we again use the co-area formula of [19, Theorem 7.7] and obtain

$$
\int_{\mathbb{B}_{\epsilon}^{l}} \mathcal{H}^{0}\left(F^{-1}(\Delta) \cap B_{u} \cap P_{1}^{-1}(v)\right) \mathrm{d} \mathcal{H}^{l}(v) \leq C \mathcal{H}^{l}\left(F^{-1}(\Delta) \cap B_{u}\right)=0
$$

since $l+d-s<l$ under our assumption. Therefore for a.e. $v \in \mathbb{B}_{\epsilon}^{l}$, $P_{1}^{-1}(v)=\emptyset$. This completes the proof for the case $\mathcal{H}^{m-s}(S)<+\infty$. The proof of the general case follows from this special case by the standard argument. We omit the details. 


\section{References}

[1] Bethuel, F.: The approximation problem for Sobolev maps between two manifolds. Acta Math. 167 (1991), 153-206.

[2] Bethuel, F. and Zheng, X.: Density of smooth functions between two manifolds in Sobolev spaces. J. Funct. Anal. 80 (1988), 60-75.

[3] Bourgain, J. And Brezis, H.: On the equation div $Y=f$ and application to control of phases. J. Amer. Math. Soc. 16 (2003), 393-426.

[4] Bourgain, J. and Brezis, H.: New estimates for elliptic equations and Hodge type systems. J. Eur. Math. Soc. (JEMS) 9 (2007), 277-315.

[5] Bredon, G.: Topology and geometry. Graduate Texts in Mathematics 139. Springer-Verlag, New York, 1993.

[6] Donaldson, S. K.: Connections, cohomology and the intersection forms of 4-manifolds. J. Differential Geom. 24 (1986), 275-341.

[7] Donaldson, S. K. And Kronheimer, P. B.: The geometry of fourManifolds. Oxford Mathematical Monographs. Oxford Science Publications. The Clarendon Press, Oxford University Press, New York, 1990.

[8] Federer, H.: Geometric measure theory. Die Grundlehren der mathematischen Wissenschaften 153. Springer-Verlag New York, New York, 1969.

[9] Giaquinta, M., Modica, G. And Souček, J.: Cartesian currents in the calculus of variations. I, II. Ergebnisse der Mathematik und ihrer Grenzgebiete 37, 38. Springer-Verlag, Berlin, 1998.

[10] Hang, F. And Lin, F. H.: Topology of Sobolev mappings. II. Acta Math. 191 (2003), 55-107.

[11] Hamburger, C.: Regularity of differential forms minimizing degenerate elliptic functionals. J. Reine Angew. Math. 431 (1992), 7-64.

[12] Husemoller, D.: Fibre Bundles, third edition. Graduate Texts in Mathematics 20. Springer-Verlag, New York, 1994.

[13] Isobe, T.: Topological and analytical properties of Sobolev bundles. I: The critical case. Ann. Global Anal. Geom. 35 (2009), 277-337.

[14] Isobe, T.: A regularity result for a class of degenerate Yang-Mills connections in critical dimensions. Forum Math. 20 (2008), 1109-1139.

[15] Kobayashi, S. and Nomizu, K.: Foundations of differential geometry. Vol. I, II. Interscience Publishers John Wiley \& Sons, New York-LondonSydney 1963, 1969.

[16] Lanzani, L. And Stein, E. M.: A note on div and curl inequalities. Math. Res. Lett. 12 (2005), 57-61.

[17] Lions, P. L.: The concentration compactness principle in the calculus of variations. The limit case. I. Rev. Mat. Iberoamericana 1 (1985), no. 1, $145-201$. 
[18] Lions, P. L.: The concentration compactness principle in the calculus of variations. The limit case. II. Rev. Mat. Iberoamericana 1 (1985), no. 2, $45-121$.

[19] Mattila, P.: Geometry of sets and measures in Euclidean spaces. Fractals and rectifiability. Cambridge Studies in Advanced Mathematics 44. Cambridge University Press, Cambridge, 1995.

[20] Meyer, Y. And RiviÈre, T.: A partial regularity result for a class of stationary Yang-Mills fields in high dimension. Rev. Mat. Iberoamericana 19 (2003), no. 1, 195-219.

[21] Nakajima, H.: Compactness of the moduli space of Yang-Mills connections in higher dimensions. J. Math. Soc. Japan 40 (1988), 383-392.

[22] Parker, T. H. and Wolfson, J. G.: Pseudo-holomorphic maps and bubble trees. J. Geom. Anal. 3 (1993), 63-98.

[23] Price, P.: A monotonicity formula for Yang-Mills fields. Manuscripta Math. 43 (1983), 131-166.

[24] Rivière, T.: Interpolation spaces and energy quantization for Yang-Mills fields. Comm. Anal. Geom. 10 (2002), 683-708.

[25] Sacks, P. And Uhlenbeck, K.: The existence of minimal immersions of 2-spheres. Ann. of Math. (2) 113 (1981), 1-24.

[26] Schoen, R. And Uhlenbeck, K.: Boundary regularity and the Dirichlet problem for harmonic maps. J. Differential Geom. 18 (1983), 253-268.

[27] SedlaceK, S.: A direct method for minimizing the Yang-Mills functional over 4-manifolds. Comm. Math. Phys. 86 (1982), 515-527.

[28] Shevchishin, V.: Limit holonomy and extension properties of Sobolev and Yang-Mills bundles. J. Geom. Anal. 12 (2002), 493-528.

[29] Simon, L.: Lectures on geometric measure theory. Proceedings of the Centre for Mathematical Analysis, Australian National University 3. Australian National Univ., Centre for Mathematical Analysis, Canberra, 1983.

[30] TaO, T. And Tian, G.: A singularity removal theorem for Yang-Mills fields in higher dimensions. J. Amer. Math. Soc. 17 (2004), no. 3, 557-593.

[31] Taubes, C. H.: Self-dual Yang-Mills connections on non-self-dual 4manifolds. J. Differential Geom. 17 (1982), 139-170.

[32] Taubes, C. H.: Self-dual connections on 4-manifolds with indefinite intersection matrix. J. Differential Geom. 19 (1984), 517-560.

[33] Taubes, C. H.: Path-connected Yang-Mills moduli spaces. J. Differential Geom. 19 (1984), 337-392.

[34] Taylor, M. E.: Partial differential equations. I-III. Applied Mathematical Sciences 115-117. Springer-Verlag, New York, 1996, 1996, 1997.

[35] Thом, R.: Quelques propriétés globales des variétés différentiables. Comment. Math. Helv. 28 (1954), 17-86.

[36] Tian, G.: Gauge theory and calibrated geometry. Ann. of Math. (2) 151 (2000), 193-268. 
[37] Uhlenbeck, K.: Regularity for a class of non-linear elliptic systems. Acta Math. 138 (1977), 219-240.

[38] Uhlenbeck, K.: Connections with $L^{p}$ bounds on curvature. Comm. Math. Phys. 83 (1982), 31-42.

[39] Uhlenbeck, K.: Removable singularities in Yang-Mills fields. Comm. Math. Phys. 83 (1982), 11-29.

[40] Uhlenbeck, K.: The Chern classes of Sobolev connections. Comm. Math. Phys. 101 (1985), 449-457.

[41] White, B.: Infima of energy functionals in homotopy classes of mappings. J. Differential Geom. 23 (1986), 127-142.

[42] White, B.: Homotopy classes in Sobolev spaces and the existence of energy minimizing maps. Acta Math. 160 (1988), 1-17.

Recibido: 6 de febrero de 2008

Takeshi Isobe

Department of Mathematics

Graduate School of Science and Engineering

Tokyo Institute of Technology

2-12-1 Oh-okayama, Meguro-ku, Tokyo 152-8551, Japan

isobe.t.ab@m.titech.ac.jp 